Bull. Soc. math. France

130 (3), 2002, p. 409-456

\title{
SURFACES KÄHLÉRIENNES DE VOLUME FINI ET ÉQUATIONS DE SEIBERG-WITTEN
}

\author{
PAR YANN ROLLIN
}

À Hélène

RÉSUMÉ. - Soit $M=\mathbb{P}(\mathcal{E})$ une surface complexe réglée. Nous introduisons des métriques de volume fini sur $M$ dons les singularités sont paramétrisées par une structure parabolique sur le fibré $\mathcal{E}$. Nous généralisons alors un résultat de Burns-de Bartolomeis et LeBrun, en montrant que l'existence de métriques kählériennes singulières, de volume fini, à courbure scalaire constante négative ou nulle sur $M$ est équivalente à une condition de polystabilité parabolique sur $\mathcal{E}$; de plus ces métriques proviennent toutes de quotients de volume fini de $\mathbb{H}^{2} \times \mathbb{C P}^{1}$. En outre nous produisons une solution des équations de Seiberg-Witten pour une métrique singulière de volume fini afin de démontrer ce théorème.

Abstract (Kähler surfaces of finite volume and Seiberg-Witten equations)

Let $M=\mathbb{P}(\mathcal{E})$ be a complex ruled surface. We introduce metrics of finite volume on $M$ whose singularities are parametrized by a parabolic structure over $\mathcal{E}$. Then, we generalise results of Burns-de Bartolomeis and Le Brun, by showing that the existence of a singular Kähler metric of finite volume and constant non positive scalar curvature on $M$ is equivalent to the parabolic polystability of $\mathcal{E}$; moreover these metrics all come from finite volume quotients of $\mathbb{H}^{2} \times \mathbb{C P}^{1}$. Therefore, we produce a solution of Seiberg-Witten equations for a singular metric $g$ of finite volume in order to prove the theorem.

Texte reçu le 31 mai 2001, accepté le 16 novembre 2001

Yann Rollin, Department of Mathematics \& Statistics, University of Edinburgh, J.C. Maxwell Building, King's Buildings, Mayfiel Road, EH9 3JZ Edinburgh, UK

E-mail : rollin@maths.ed.ac.uk •Url : www.maths.ed.ac.uk/ ${ }^{\sim}$ rollin

Classification mathématique par sujets (2000). - 53C20, 53C24, 32L05, 14D21.

Mots clefs. - Seiberg-Witten, surfaces réglées, métriques de Kähler, fibrés paraboliques, stabilité.

The author was supported by an EDGE grant, Research Training Network HPRN-CT-200000101, European Human Potential Programme. 


\section{Introduction}

L'existence de métriques de Kähler à courbure scalaire constante sur les surfaces complexes est un problème ouvert dans lequel de récentes avancées mettent en évidence l'importance de la notion de stabilité (voir [6], [4] et [5]).

Dans le cas d'une surface géométriquement réglée de la forme $M=\mathbb{P}(\mathcal{E})$, où $\mathcal{E}$ est un fibré holomorphe au-dessus d'une surface de Riemann hyperbolique compacte $\bar{\Sigma}$, Burns et de Bartolomeis ont démontré que les seules métriques kählériennes à courbure scalaire $s=0$ sont des produits locaux; par le théorème de Narashiman-Seshadri, cette condition est équivalente à la polystabilité de $\mathcal{E}$. Puis ce résultat a été généralisé par Le Brun au cas $s<0$ en utilisant la théorie de Seiberg-Witten.

Mehta et Seshadri étendent les résultats de [14] en volume fini grâce à la théorie des fibrés paraboliques et une condition de polystabilité parabolique (pour une démonstration « à la Donaldson » [8] de leur résultat, cf. [2]). D'après [12], ces fibrés correspondent, en rang 2, aux cas où la surface réglée $M$ est un quotient de volume fini $\Sigma \times{ }_{\rho} \mathbb{C P}^{1}$, provenant d'une représentation $\rho: \pi_{1}(\Sigma) \rightarrow$ $\mathrm{PU}(2)$, où $\Sigma=\bar{\Sigma} \backslash\left\{P_{i}\right\}$ est une surface de Riemann de type hyperbolique obtenue en enlevant un nombre fini de points appelés points paraboliques à une surface de Riemann compacte. En munissant le premier facteur de la métrique hyperbolique à courbure -1 et le second de la métrique de Fubini-Study à courbure $c>0$, on en déduit une métrique kählérienne $\hat{g}$ à courbure scalaire $s=$ $2(c-1)$ sur $M$. Dans des coordonnées locales adaptées $(t, \theta, u) \in \mathbb{R} \times S^{1} \times \mathbb{C P}^{1}$ sur les bouts de $M$, où $u$ est une coordonnée affine sur $\mathbb{C P}^{1}$, la métrique $\hat{g}$ est donnée par

$$
\hat{g}=\mathrm{d} t^{2}+\mathrm{e}^{-2 t} \mathrm{~d} \theta^{2}+\frac{4 / c}{\left(1+|u|^{2}\right)^{2}}|\mathrm{~d} u-i \alpha u \mathrm{~d} \theta|^{2},
$$

où $\alpha \in[0,1[$ est le poids de cette singularité appelée bout parabolique. Nous allons étendre les résultats de [6] et [4] à ce cadre de volume fini en supposant que les métriques considérées sont asymptotiques au sens $C^{2}$ au modèle local défini par (1).

ThÉORÈmE A. - Soit $\mathcal{E} \longrightarrow \bar{\Sigma}$ un fibré parabolique holomorphe tel que $\Sigma=$ $\bar{\Sigma} \backslash\left\{P_{i}\right\}$ soit hyperbolique. Soit $M=\mathbb{P}(\mathcal{E})$ la surface complexe réglée associée restreinte au-dessus de $\Sigma$. Alors $M$ admet une métrique kählérienne $g^{\mathrm{K}}$ à courbure scalaire constante $s \leq 0$ asymptotique au modèle local si et seulement si le fibré $\mathcal{E}$ est paraboliquement polystable. Dans ce cas, la métrique $g^{\mathrm{K}}$ se déduit à un biholomorphisme et une constante près du modèle $\left(\Sigma \times_{\rho} \mathbb{C P}^{1}, \hat{g}\right)$ où $\rho: \pi_{1}(\Sigma) \longrightarrow \mathrm{PU}(2)$ est une représentation associée au fibré parabolique polystable $\mathcal{E}$.

REMARQUE. - Si $g^{\mathrm{K}}$ admet au moins une singularité, son comportement asymptotique impose que la constante du théorème soit égale à 1 .

TOME $130-2002-\mathrm{N}^{\mathrm{O}} 3$ 
On rencontre la difficulté majeure de la démonstration dans le cas $s<0$, où pour procéder suivant la méthode de Le Brun on doit extraire une solution $(A, \psi)$, suffisamment régulière, des équations de Seiberg-Witten

$$
D_{A} \psi=0, \quad F_{A}^{+}=q(\psi)
$$

pour une métrique $g$ asymptotique au modèle local et la structure $\operatorname{spin}^{c}$ canonique de $M$. En l'absence de singularités, LeBrun utilise la théorie des invariants de Seiberg-Witten sur les variétés compactes, ce qui n'est pas le cas de $M$. Pour traiter ce problème, on montre dans la section 2 que lorsque les poids des singularités sont rationnels, $M$ admet une compactification orbifold $\bar{M}=M \cup D$, où $D$ est une réunion de diviseurs de la forme $\mathbb{C P}^{1} / \mathbb{Z}_{q}$, sur laquelle on peut approximer $g$ par une suite de métriques lisses $g_{j}$. On définit alors les équations de Seiberg-Witten perturbées sur $\bar{M}$ pour chaque métrique $g_{j}$ par

$$
D_{A} \psi=0, \quad\left(F_{A}+2 i \pi \varpi_{j}\right)^{+}=q(\psi),
$$

où les perturbation $\varpi_{j}$ se concentrent vers le courant d'intégration sur $D$. En calculant l'invariant des équations de Seiberg-Witten qui dépend d'une certaine condition de chambre pour la métrique $g_{j}$, nous obtiendrons une suite de solutions $\left(A_{j}, \psi_{j}\right)$ que nous ferons converger vers une solution $(A, \psi)$ des équations non perturbée pour la métrique limite $g$ grâce au théorème suivant :

ThÉORÈme B. - Soit $g$ une métrique sur $M$ asymptotique au modèle local $\hat{g}$ avec des poids $\alpha\left(P_{i}\right)$ rationnels et soit $g_{j}$ la suite d'approximation de g sur $\bar{M}$. Soit $\left(A_{j}, \psi_{j}\right)$ une suite de solutions des équations de Seiberg-Witten perturbées associées à la structure $\operatorname{spin}^{c}$ canonique induite par la structure complexe et aux métriques $g_{j}$ sur $\bar{M}$. Alors quitte à faire des changements de jauge et à extraire une sous-suite, $\left(A_{j}, \psi_{j}\right)$ converge au sens $C^{\infty}$ sur tout compact de $M$ vers une solution des équations non perturbées $(A, \psi)$ pour la métrique $g$ vérifiant

- $A=\hat{A}+a$ avec $a \in L_{1}^{2}(g)$,

- $\psi \in L_{1}^{2}(g)$ relativement à $\nabla_{\hat{A}}$,

où $\hat{A}$ est une connexion induite par le modèle local kählérien $\hat{g}$ sur le fibré déterminant $L=K_{M}^{-1}$ de la structure $\operatorname{spin}^{c}$ canonique.

REMARques. - On pourra se référer à [3] pour une autre application des équations de Seiberg-Witten à des métriques d'Einstein de volume fini.

- Il serait naturel que l'existence de la solution $(A, \psi)$ obtenue par ce théorème soit assurée par une théorie de Seiberg-Witten développée directement pour les métriques asymptotiques au modèle local. Notons que la construction de l'espace des modules correspondant et la question de sa compacité posent des problèmes techniques importants que notre méthode ne résout pas ( $c f$. par exemple [11] pour des métriques asymptotiquement plates). 
- La perturbation $\varpi_{j}$ est nécessaire pour que la connexion $A$ soit définie sur le «bon fibré » et s'explique au niveau des équations par le fait que la métrique $g$ apparaît comme une limite de métriques sur la variété compacte moins une bulle de courbure positive.

La première étape dans la démonstration du théorème $\mathrm{B}$ ( $c f$. section 3 ) consiste à développer une théorie de Hodge pour les métriques $g$ et $g_{j}$ via des inégalités de Poincaré uniformes. À l'aide d'un lemme de Poincaré local près de $D$ pour la cohomologie $L^{2}$, on démontre un isomorphisme

$$
\mathrm{H}_{L^{2}}^{k}(M) \simeq \mathrm{H}_{\mathrm{DR}}^{k}(\bar{M}),
$$

puis on démontre pour $k=1$ ou 2 que les représentant $g_{j}$-harmoniques d'une classe de cohomologie convergent vers le représentant $g$-harmonique $L^{2}$ sur tout compact de $M$. À partir de là on possède tous les outils nécessaires pour faire converger les connexions, pour démontrer le théorème au $\S 4.2$ puis pour calculer l'invariant de Seiberg-Witten des métriques $g_{j}$ au $\S 4.5$. Dans le cas de poids irrationnels, on n'a plus de compactification orbifold adéquate et on doit faire une convergence « en deux temps » en commençant par approximer les poids irrationnels par des poids rationnels ( $c f . \S 4.4)$.

Sur les éclatements de surfaces réglées. - La structure parabolique, en chaque point $P$ non trivial d'un fibré $\mathcal{E}$ ( $c f$. $§ 2.2$ pour la définition), détermine une droite complexe de $\mathcal{E}_{P}$, donc un point $Q$ de de la surface compacte $\widehat{M}=\mathbb{P}(\mathcal{E})_{\Sigma}$ et un poids $\alpha=\alpha_{2}-\alpha_{1}$. Le problème d'existence de métriques de Kähler à courbure scalaire constante sur l'éclatement $\widetilde{M}$ de $\widehat{M}$ aux points $Q_{i}$ a été abordé par Le Brun et Singer lorsque $\widehat{M}$ possède des champs de vecteurs holomorphes périodiques et $s=0$ : suivant [5], de telles métriques existent si et seulement si $\mathcal{E}$ est paraboliquement polystable.

La forme de Kähler $\omega$ de la métrique $\hat{g}$, bien que singulière sur $\widehat{M}$, correctement interprétée comme un courant positif ( $c f .[7])$, se relève en un courant positif $\widehat{\omega}$ appelé transformée stricte de $\omega$ sur l'éclatement $\widetilde{M}$ tel que

$$
0<\alpha=\frac{\widehat{\omega} \cdot[E]}{\widehat{\omega} \cdot[F]},
$$

avec $E$ le diviseurs exceptionel au point $Q$ et $F$ une fibre générique. Cette identité est précisément celle vérifiée par les classes de Kähler considérées dans [5] et ceci constitue une indication supplémentaire mettant en évidence le lien entre la notion de stabilité et le problème d'existence de métriques kählériennes à courbure scalaire constante sur les surfaces complexes.

Je tiens à remercier tout particulièrement Olivier Biquard pour l'ensemble des discussions que j'ai eues avec lui sur ce sujet.

TOME $130-2002-\mathrm{N}^{\mathrm{O}} 3$ 


\section{Surfaces kählériennes réglées modèles}

2.1. Un exemple fondamental. - Voici tout d'abord une famille de surfaces complexes réglées construites à partir de représentations unitaires du groupe fondamental d'une surface de Riemann de volume fini. Ces exemples sont essentiels dans la théorie des fibrés parabolique stables comme nous le verrons au $\S 2.2 .2$ où nous citerons le théorème important de Mehta-Seshadri; en outre ces exemples possèdent des métriques kählériennes « modèles » à courbure scalaire constante avec des singularités que nous étudierons précisément au $§ 2.3$ et que nous appellerons bouts paraboliques.

2.1.1. Surfaces de Riemann hyperboliques de volume fini. - Soit $\Gamma$ un sousgroupe discret de $\operatorname{PSL}(2, \mathbb{R})$ agissant librement et avec covolume fini sur le demi plan de Poincaré $\mathbb{H}^{2}=\{\xi \in \mathbb{C} ; \operatorname{Im} \xi>0\}$; le quotient est une surface de Riemann $\Sigma=\mathbb{H}^{2} / \Gamma$, de groupe fondamental $\Gamma$, munie de la métrique kählérienne $g^{\Sigma}$ complète de volume fini à courbure -1 induite par la métrique de Lobachevsky. Le groupe $\Gamma$ agit également sur le bord à l'infini $\partial_{\infty} \mathbb{H}^{2}$ du demiplan de Poincaré. Puisque $\Gamma$ agit avec covolume fini, le stabilisateur d'un point du bord est soit trivial, soit, pour un nombre fini de points $P$ appelés points paraboliques, égal à $\langle\tau\rangle \simeq \mathbb{Z}$, où $\tau$ est un élément parabolique de $\operatorname{PSL}(2, \mathbb{R})$. On peut donc, quitte à conjuguer $\tau$ par une homographie, supposer qu'il est donné par $\tau: \xi \mapsto \xi+u$ où $\xi \in \mathbb{H}^{2}$ et $u \in \mathbb{R}$; pour simplifier on supposera même que $u=2 \pi$. Au voisinage de $P$, le quotient $\mathbb{H}^{2} / \Gamma$ est isomorphe à $I_{a} /\langle\tau\rangle$, où $I_{a}=\{\xi \in \mathbb{C} ; \operatorname{Im} \xi>a\}$ avec $a>0$ suffisamment grand. On définit alors un isomorphisme entre le disque épointé $\Delta_{a}^{*}=\left\{z \in \mathbb{C} ; 0<|z|<\mathrm{e}^{-a}\right\}$ et $I_{a} /\langle\tau\rangle$ par

$$
I_{a} /\langle\tau\rangle \longrightarrow \Delta_{a}^{*}, \quad \xi \longmapsto z=\mathrm{e}^{i \xi}
$$

en utilisant le plongement holomorphe $\Delta_{a}^{*} \subset \Delta_{a}$, ce qui revient à ajouter le point $P$ correspondant à 0 dans le modèle du disque, on obtient finalement la compactification holomorphe $\bar{\Sigma}=\Sigma \cup\left\{P_{i}\right\}_{1<i<k}$.

En partant de la métrique de Lobachevsky donnée par

$$
g^{\mathbb{H}^{2}}=\frac{|\mathrm{d} \xi|^{2}}{|\xi-\bar{\xi}|^{2}},
$$

on calcule aisément la métrique induite sur le disque épointé :

$$
g^{\Delta^{*}}=\frac{|\mathrm{d} z|^{2}}{|z|^{2} \cdot \ln ^{2}|z|} .
$$

Cette métrique possède une singularité en 0 appelée cusp. Nous utiliserons souvent un autre système de coordonnées locales $(t, \theta) \in \mathbb{R} \times \mathbb{R} / 2 \pi \mathbb{Z}$ sur les bouts de $\Sigma$ défini par $z=r \mathrm{e}^{i \theta}$ et $t=\ln (-\ln |z|)$; dans ces coordonnées

$$
g^{\Delta^{*}}=\mathrm{d} t^{2}+\mathrm{e}^{-2 t} \mathrm{~d} \theta^{2} .
$$

BULlETiN DE LA SOCIÉtÉ MATHÉMATIQUE DE FRANCE 
Réciproquement ( $c f .[9])$, si $\bar{\Sigma}$ est une surface de Riemann compacte avec $k$ points paraboliques marqués $\left(P_{i}\right)$, alors $\Sigma=\bar{\Sigma} \backslash\left\{P_{i}\right\}$ est hyperbolique si et seulement si $2 g(\bar{\Sigma})-2+k>0$ et dans ce cas elle admet une unique métrique kählérienne $g^{\Sigma}$ de courbure -1 avec des cusps aux points paraboliques.

2.1.2. Représentations et surfaces réglées. - Soit $\rho: \Gamma \longrightarrow \mathrm{U}(2)$ une représentation unitaire du groupe fondamental de $\Sigma$. On en déduit une action de $\Gamma$ sur $\mathbb{H}^{2} \times \mathbb{C}^{2}$ donnée par $\sigma \cdot(\xi, u)=(\sigma \cdot \xi, \rho(\sigma) \cdot u)$ pour $\sigma \in \Gamma$ et $(\xi, \mu) \in \mathbb{H}^{2} \times \mathbb{C}^{2}$. $\mathrm{Au}$ quotient, on obtient un fibré vectoriel holomorphe $\mathcal{E}=\mathbb{H}^{2} \times_{\rho} \mathbb{C}^{2}$ au-dessus de $\Sigma$. On en déduit une surface complexe réglée $M=\mathbb{P}(\mathcal{E})=\mathbb{H}^{2} \times{ }_{\rho} \mathbb{C P}^{1}$ où $\rho$ désigne par abus de langage la représentation projective induite. La sphère de Riemann $\mathbb{C P}^{1}$ est munie d'une métrique kählérienne à courbure sectionnelle $c>0$, appelée métrique de Fubini-Study, définie dans des coordonnées homogènes $[u: v]$ par

$$
\omega^{\mathrm{FS}}=\frac{2 i}{c} \partial \bar{\partial} \ln \left(|u|^{2}+|v|^{2}\right)
$$

dans la carte $v=1$, on obtient alors

$$
g^{\mathrm{FS}}=\frac{4 / c}{\left(1+|u|^{2}\right)^{2}}|\mathrm{~d} u|^{2} .
$$

La métrique produit $g^{\mathbb{H}^{2}} \oplus g^{\mathrm{FS}}$ sur $\mathbb{H}^{2} \times \mathbb{C P}^{1}$ est kählérienne à courbure scalaire constante $s=2(c-1)$, car c'est un produit riemannien de métriques kählériennes à courbures scalaires constantes. Le groupe d'isométries de $\left(\mathbb{C P}^{1}, g^{\mathrm{FS}}\right)$ est égal à $\mathrm{PU}(2)$, donc $\Gamma$ agit isométriquement sur $\mathbb{H}^{2} \times \mathbb{C P}^{1}$. On en déduit par quotient une métrique kählérienne à courbure scalaire constante $\hat{g}$ sur $M$ appelée métrique modèle. En outre, $\hat{g}$ est de volume fini car c'est un produit local donc $\operatorname{vol}(M)=\operatorname{vol}^{\Sigma}(\Sigma) \times \operatorname{vol}^{\mathrm{FS}}\left(\mathbb{C P}^{1}\right)<\infty$.

2.2. Fibrés paraboliques. - Une question naturelle se pose maintenant si nous voulons fabriquer des métriques de Kähler de volume fini à courbure scalaire constante sur les surfaces complexes réglées comme dans l'exemple précédent : comment reconnaître celles qui sont obtenues à partir de représentation projective unitaire du groupe fondamental d'une surface de Riemann de volume fini ? La réponse à nous est donnée par la théorie des fibrés paraboliques stables développée par Mehta et Seshadri; nous rappelons dans cette partie des éléments de la théorie, mais pour de plus amples détails nous renvoyons le lecteur à $[12]$.

2.2.1. Structures paraboliques. - Le fibré hermitien plat $\mathcal{E}=\mathbb{H}^{2} \times{ }_{\rho} \mathbb{C}^{2}$ admet un prolongement holomorphe au-dessus de $\bar{\Sigma}$ sur lequel la métrique hermitienne est singulière; en considérant les morphismes bornés de fibrés paraboliques, on voit apparaître naturellement la notion de structure parabolique. 
DÉFINITION 1 (structures paraboliques). - Une structure parabolique sur un fibré vectoriel complexe $\mathcal{E} \longrightarrow \bar{\Sigma}$ au-dessus d'une surface de Riemann compacte est la donnée d'un ensemble fini points paraboliques $P_{i} \in \bar{\Sigma}$, de filtrations audessus des points paraboliques

$$
\mathcal{E}_{P_{i}}=\mathcal{F}_{P_{i}}^{1} \supsetneq \cdots \supsetneq \mathcal{F}_{P_{i}}^{k_{i}+1}=0,
$$

et de poids associés à la filtration $0 \leq \alpha_{1}\left(P_{i}\right)<\cdots<\alpha_{k_{i}}\left(P_{i}\right)<1$; on appelle multiplicité d'un poids $\alpha_{j}\left(P_{i}\right)$ l'entier $d_{j}\left(P_{i}\right)=\operatorname{dim} \mathcal{F}_{P_{i}}^{j}-\operatorname{dim} \mathcal{F}_{P_{i}}^{j+1}$. Un fibré muni d'une structure parabolique est appelé un fibré parabolique.

Remarque. - Pour les fibrés paraboliques de rang 2, on a soit $k_{i}=2$, soit $k_{i}=1$. Dans ce dernier cas, la structure parabolique au point $P_{i}$ est réduite à une filtration triviale et à un unique poids de multiplicité 2 . Un tel point parabolique est appelé un point trivial de la structure parabolique.

Dans une base orthonormée $\left(\epsilon_{1}, \epsilon_{2}\right)$ bien choisie, on a

$$
\rho(\tau)=\left(\begin{array}{cc}
\mathrm{e}^{2 i \pi \alpha_{1}} & 0 \\
0 & \mathrm{e}^{2 i \pi \alpha_{2}}
\end{array}\right) \quad \text { avec } \quad 0 \leq \alpha_{1} \leq \alpha_{2}<1
$$

on en déduit une base de sections locales holomorphes $\left(s_{1}, s_{2}\right)$ de $\mathbb{H}^{2} \times{ }_{\rho} \mathbb{C P}^{1}$ en $P$ telle que la métrique hermitienne s'écrive

$$
h=\left(\begin{array}{cc}
|z|^{2 \alpha_{1}} & 0 \\
0 & |z|^{2 \alpha_{2}}
\end{array}\right) .
$$

On définit alors une structure parabolique sur $\mathcal{E}$ comme suit : en un point parabolique $P$ de $\bar{\Sigma}$ tel que $\alpha_{1}<\alpha_{2}$, on pose $\mathcal{F}_{P}^{2}=\mathbb{C} s_{2}(P), \alpha_{1}(P)=\alpha_{1}$ et $\alpha_{2}(P)=\alpha_{2}$. Si $\alpha_{1}=\alpha_{2}$, on décide que $P$ est un point trivial de la structure parabolique avec un unique poids $\alpha_{1}(P)=\alpha_{1}$.

Avec cette définition de structure parabolique, on peut voir facilement que les isomorphisme de $\mathcal{E}$ bornés relativement à la métrique hermitienne sont exactement ceux qui respectent les filtrations au-dessus des points paraboliques.

2.2.2. Stabilité parabolique et théorème de Mehta-Seshadri. — Dans le $§ 2.2 .2$, $\mathcal{E} \rightarrow \bar{\Sigma}$ désignera un fibré parabolique holomorphe. Son degré parabolique et sa pente sont définis respectivement par

$$
\operatorname{deg} \operatorname{par} \mathcal{E}=\operatorname{deg} \mathcal{E}+\sum_{i, j} d_{j}\left(P_{i}\right) \cdot \alpha_{j}\left(P_{i}\right), \quad \mu(\mathcal{E})=\frac{\operatorname{deg} \operatorname{par} \mathcal{E}}{\operatorname{rang} \mathcal{E}} .
$$

Si $\mathcal{E}=\mathcal{L}_{1} \oplus \mathcal{L}_{2}$ est une somme de deux fibrés paraboliques en droites complexes, alors $\mathcal{E}$ possède une structure parabolique induite. On dira que $\mathcal{E}$ est paraboliquement décomposable s'il admet une décomposition holomorphe en une somme directe de fibrés parabolique $\mathcal{E}=\mathcal{L}_{1} \oplus \mathcal{L}_{2}$ qui est de plus compatible avec la structure parabolique de $\mathcal{E}$.

Un fibré parabolique $\mathcal{E} \rightarrow \bar{\Sigma}$, de rang 2 , est dit paraboliquement stable (resp. paraboliquement semi-stable) si tout sous-fibré holomorphe en droites 
complexes $\mathcal{L}$ muni de la structure parabolique induite vérifie $\mu(\mathcal{L})<\mu(\mathcal{E})$ (resp. $\mu(\mathcal{L}) \leq \mu(\mathcal{E}))$. Le fibré $\mathcal{E}$ sera dit paraboliquement polystable s'il est soit paraboliquement stable, soit à la fois paraboliquement semi-stable et paraboliquement décomposable.

Lorsque $\mathcal{E} \rightarrow \bar{\Sigma}$ est un fibré parabolique tel que $\Sigma=\bar{\Sigma} \backslash\left\{P_{i}\right\}$ soit hyperbolique, on a le théorème important de Mehta et Seshadri (cf. [12], th. 4.1) d'après lequel $\mathcal{E}$ est polystable de pente $\mu=0$ si et seulement si il est plat et donné à isomorphisme parabolique près par une représentation $\rho: \pi_{1}(\Sigma) \longrightarrow \mathrm{U}(2)$.

Lorsque deg par $\mathcal{E} \neq 0$, on peut se ramener au cas du degré parabolique nul de la façon suivante : soit $\mathcal{L} \rightarrow \bar{\Sigma}$, un fibré en droites complexes de degré très négatif, de sorte que deg $\operatorname{par}(\mathcal{E} \otimes \mathcal{L})<0$. Notons que les surfaces $\mathbb{P}(\mathcal{E})$ et $\mathbb{P}(\mathcal{E} \otimes \mathcal{L})$ sont isomorphes. Dans un deuxième temps, on ajoute des points paraboliques triviaux $Q_{k} \in \Sigma$ à la structure parabolique de sorte que le nouveau fibré parabolique $\mathcal{E}^{\prime}$ vérifie deg par $\mathcal{E}^{\prime}=\operatorname{deg} \operatorname{par}(\mathcal{E} \otimes \mathcal{L})+\sum_{k} 2 \alpha_{1}\left(Q_{k}\right)=0$. En choisissant bien les poids, on obtient un fibré de degré parabolique 0 et (cf. [15]) le fibré $\mathcal{E}^{\prime}$ est paraboliquement stable (resp. semi-stable, polystable) si et seulement si le fibré $\mathcal{E}$ est paraboliquement stable (resp. semi-stable, polystable).

2.2.3. Stabilité et métriques modèles. - Soit $\mathcal{E} \rightarrow \bar{\Sigma}$ un fibré parabolique polystable et $M=\mathbb{P}(\mathcal{E})_{\mid \Sigma}$ la surface réglée associée. En supposant $\Sigma$ hyperbolique, on sait que $\Sigma$ admet une unique métrique kählérienne $g^{\Sigma}$ à courbure sectionnelle -1 avec des cusps aux points $P_{i}$. On peut quitte à ajouter des points paraboliques triviaux $Q_{i}$ supposer que $\operatorname{deg} \operatorname{par} \mathcal{E}=0$ sans changer la stabilité parabolique du fibré.

Alors, d'après le théorème de Mehta et Seshadri, il existe une représentation projective unitaire $\rho: \pi_{1}\left(\Sigma \backslash\left\{Q_{i}\right\}\right) \rightarrow \mathrm{U}(2)$ telle que $\mathcal{E} \simeq \mathbb{H}^{2} \times{ }_{\rho} \mathbb{C}^{2}$. Il est alors facile de voir que la métrique hermitienne, singulière aux points $Q_{i}$, induit une métrique de Fubini-Study sur la fibre qui est lisse sur $M$ tout entière. On obtient alors suivant $\S 2.1$ une métrique kählérienne $\hat{g}$ à courbure scalaire constante sur $M$.

REMARQUE. - La construction que nous venons de faire montre que nous pouvons facilement nous ramener au cas des fibrés paraboliques de pente 0 sans ajouter de singularités à la métrique $\hat{g}$. Par la suite, nous supposerons pour simplifier que tous les fibrés paraboliques sont de pente nulle. De plus, nous ferons comme si les structure paraboliques étaient concentrées au-dessus d'un unique point parabolique, toutes les constructions s'étendant trivialement au cas de plusieurs points.

Réciproquement, il est naturel de se demander si l'existence d'une métrique kählérienne à courbure scalaire constante sur $M$ avec des singularités de volume fini implique la stabilité du fibré parabolique $\mathcal{E}$ de départ. Nous allons résoudre cette question dans le théorème 2.1, mais pour l'énoncer, nous devons introduire au préalable une notion de modèle local.

TOME $130-2002-\mathrm{N}^{\mathrm{O}} 3$ 
2.3. Le modèle local. — Nous allons maintenant nous concentrer sur l'étude locale de la métrique $\hat{g}$ au voisinage d'un point parabolique.

La métrique $\hat{g}$ que nous avons définie sur la surface réglée $M$ tout entière lorsque $\mathcal{E}$ est paraboliquement polystable, peut être définie localement en général sur un fibré parabolique. Pour commencer, on choisit une trivialisation locale de $\mathcal{E}$ par une base de sections holomorphes $\left(s_{1}, s_{2}\right)$ définies au voisinage d'un point parabolique $P$ compatible avec la filtration parabolique. Soit $z$ une coordonnée locale holomorphe telle que $z(P)=0$ et telle que la métrique sur $\Sigma$ s'exprime sous la forme

$$
g^{\Sigma}=\frac{|\mathrm{d} z|^{2}}{|z|^{2} \cdot \ln ^{2}|z|} .
$$

On définit alors dans la base $\left(s_{1}, s_{2}\right)$ une métrique hermitienne sur $\mathcal{E}$ adaptée à la structure parabolique par (5). On en déduit une base orthonormée de sections locales en $P$ données par $e_{i}=s_{i} /|z|^{\alpha_{i}}$ et on calcule la connexion de Chern associée à $h$ dans la trivialisation $\left(s_{i}\right)$ :

$$
\nabla^{h}=\mathrm{d}+h^{-1} \partial h=d+\left(\begin{array}{cc}
\alpha_{1} & 0 \\
0 & \alpha_{2}
\end{array}\right) \frac{\mathrm{d} z}{z}
$$

ce qui donne dans les coordonnées induites par la base orthonormales $\left(e_{i}\right)$,

$$
\nabla^{h}=\mathrm{d}+i\left(\begin{array}{cc}
\alpha_{1} & 0 \\
0 & \alpha_{2}
\end{array}\right) \mathrm{d} \theta
$$

Cette connexion est plate, avec une singularité logarithmique au point $P$. On retrouve dans cette expression locale l'holonomie de la connexion de Chern égale à $\operatorname{diag}\left(\exp \left(-2 i \pi \alpha_{1}\right), \exp \left(-2 i \pi \alpha_{2}\right)\right)$ autour du point $P$. En notant $H_{x}$ la distribution horizontale de la connexion induite sur le $\mathbb{C P}^{1}$-fibré $M \rightarrow \Sigma$, on a une décomposition $T_{x} M=H_{x} \oplus T_{x} \mathbb{P}\left(\mathcal{E}_{\pi(x)}\right)$. Comme la connexion de Chern respecte par définition la métrique hermitienne sur $\mathcal{E}$, on en déduit que la distribution $\left(H_{x}\right)$ est invariante par les isométries de la métrique de FubiniStudy sur les fibres de $M \rightarrow \Sigma$.

On définit alors localement la métrique riemannienne $\hat{g}$ en prenant la métrique de Fubini-Study sur l'espace tangent aux fibres et l'image réciproque de $g^{\Sigma}$ sur $H_{x} \simeq T_{x} \Sigma$. Comme de plus la connexion est plate, la distribution horizontale $H_{x}$ est intégrable, et $\hat{g}$ apparaît comme un produit riemannien local $\mathbb{H}^{2} \times \mathbb{C P}^{1}$. On dit que $\hat{g}$ est adaptée à la structure parabolique de $\mathcal{E}$.

2.3.1. Revêtement et modèle local. — En reprenant les notations de §2.2.1, nous disposons de plusieurs coordonnées locales définies au voisinage de $P$ : la coordonnée $\xi \in \mathbb{H}^{2}$, la coordonnée $z \in \Delta_{a}$ définie au voisinage de $P$, les coordonnées $(\xi,(\tilde{u}, \tilde{v}))$ sur $\mathbb{H}^{2} \times \mathbb{C}^{2}$ induites par la base canonique $\left(\epsilon_{i}\right)$ de $\mathbb{C}^{2}$ et les coordonnées $(z,(u, v))$ sur $\mathcal{E}$ induites par la base orthonormée de sections locales $\left(e_{i}\right)$ de $\mathcal{E}$. On en déduit des coordonnées locales homogènes sur $\mathbb{H}^{2} \times \mathbb{C P}^{1}$ 
et $\mathbb{P}(\mathcal{E})$ et on a le revêtement holomorphe riemannien

$$
\begin{aligned}
p: I_{a} \times \mathbb{C P}^{1} & \longrightarrow \Delta_{a}^{*} \times \mathbb{C P}^{1}, \\
(\xi,[\tilde{u}: \tilde{v}]) & \longmapsto\left(\mathrm{e}^{i \xi},\left[\mathrm{e}^{-i \alpha_{1} \operatorname{Re} \xi} \tilde{u}: \mathrm{e}^{-i \alpha_{2} \operatorname{Re} \xi} \tilde{v}\right]\right),
\end{aligned}
$$

avec

$$
\tau \cdot(\xi,[\tilde{u}: \tilde{v}])=\left(\xi+2 \pi,\left[\mathrm{e}^{2 i \pi \alpha_{1}} \tilde{u}: \mathrm{e}^{2 i \pi \alpha_{2}} \tilde{v}\right]\right)
$$

qui engendre le groupe d'automorphismes $\langle\tau\rangle \simeq \mathbb{Z}$ du revêtement $p$.

$\mathrm{Si}$ on se place par exemple dans les coordonnées locales de projection stéréographique $\tilde{v}=1$ et $v=1$, on obtient l'expression de $p$ :

$$
p(\xi, \tilde{u})=\left(\mathrm{e}^{i \xi}, \mathrm{e}^{i \alpha \operatorname{Re} \xi} \tilde{u}\right) \quad \text { où } \quad \alpha=\alpha_{2}-\alpha_{1} .
$$

On en déduit que $\mathrm{e}^{i \alpha \operatorname{Re} \xi} \mathrm{d} \tilde{u}=p^{*} \mathrm{~d} u-i \alpha u \mathrm{~d} \theta$. Or la métrique standard sur le revêtement s'écrit

$$
p^{*} \hat{g}=g^{\mathbb{H}^{2}} \oplus g^{\mathrm{FS}}=\frac{|\mathrm{d} \xi|^{2}}{|\xi-\bar{\xi}|^{2}}+\frac{4 / c}{\left(1+|u|^{2}\right)^{2}}|\mathrm{~d} \tilde{u}|^{2} .
$$

d'où

$$
\hat{g}=\frac{|\mathrm{d} z|^{2}}{(|z| \cdot \ln |z|)^{2}}+\frac{4 / c}{\left(1+|u|^{2}\right)^{2}}|\mathrm{~d} u-i \alpha u \mathrm{~d} \theta|^{2} .
$$

On peut maintenant dégager la notion de bout parabolique sur une variété.

DÉFINITION 2. - Le groupe $\mathbb{Z} \simeq\langle\tau\rangle$ a une action libre holomorphe et isométrique sur la variété complexe kählérienne $\mathbb{H}^{2} \times \mathbb{C P}^{1}$ munie de sa métrique standard à courbure scalaire $2(c-1)$ définie par

$$
\tau \cdot(\xi,[\tilde{u}: \tilde{v}])=\left(\xi,\left[\tilde{u} \mathrm{e}^{2 i \pi \alpha_{1}}: \tilde{v} \mathrm{e}^{2 i \pi \alpha_{2}}\right]\right) .
$$

Le quotient $\left(I_{a} \times \mathbb{C P}^{1}\right) / \mathbb{Z}$ est par conséquent une variété kählérienne munie de la métrique de Kähler quotient $\hat{g}$ à courbure scalaire $2(c-1)$ et de volume fini. Ce quotient sera appelé le bout parabolique associé aux poids $\alpha_{1}, \alpha_{2}$. Nous le noterons $\mathcal{B}_{a}^{\alpha_{1}, \alpha_{2}}$, ou bien $\mathcal{B}$ lorsque aucune ambiguïté n'est possible.

Par définition, un bout parabolique est muni de coordonnées adaptées $(z,[u: v]) \in \Delta_{a}^{*} \times \mathbb{C P}^{1}$ dans lesquelles le revêtement canonique $p: I_{a} \times \mathbb{C P}^{1} \rightarrow \mathcal{B}$ est donné par (7) et la métrique $\hat{g}$ est alors donnée localement par (9).

Dans des coordonnées adaptées, la projection suivant le premier facteur,

$$
\pi: \mathcal{B} \simeq \Delta_{a}^{*} \times \mathbb{C P}^{1} \longrightarrow \Delta_{a}^{*}
$$

TOME $130-2002-\mathrm{N}^{\mathrm{O}} 3$ 
est holomorphe et donne au bout parabolique une structure de $\mathbb{C P}^{1}$-fibré et on a clairement le diagramme commutatif

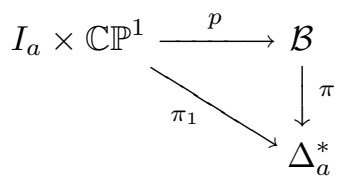

où $\pi_{1}$ est induite par le revêtement canonique $I_{a} \rightarrow \Delta_{a}^{*}$.

REMARQUE. - La forme volume de la métrique de Fubini-Study sur $\mathbb{H}^{2} \times \mathbb{C P}^{1}$ passe au quotient et donne une 2-forme fermée sur le bout parabolique que nous noterons vol $\mathbb{C P}^{1}$.

En remplaçant la coordonnée $z$ par $(t, \theta)$, la métrique modèle apparaît comme un produit tordu

$$
\hat{g}=\mathrm{d} t^{2}+\mathrm{e}^{-2 t} \mathrm{~d} \theta^{2}+\frac{4 / c}{\left(1+|u|^{2}\right)^{2}}|\mathrm{~d} u-i \alpha u \mathrm{~d} \theta|^{2}=\mathrm{d} t^{2}+\hat{g}_{t},
$$

où $\hat{g}_{t}$ est une métrique sur $N \simeq S^{1} \times \mathbb{C P}^{1}$ que nous appellerons la tranche du bout parabolique.

La métrique sur $\mathbb{H}^{2} \times \mathbb{C P}^{1}$ dégénère dans la direction $\partial_{x}=\mathrm{d} x^{\sharp}$ où $\xi=$ $x+i y$ et où $\mathrm{d} x^{\sharp}$ désigne le dual suivant la métrique de $\mathrm{d} x$. Ce champ de vecteurs est invariant sous l'action de $\tau$. Par conséquent $\partial_{x}=\mathrm{d} x^{\sharp}$ passe au quotient et nous donne un champ de vecteurs noté $\mathcal{X}_{\theta}$ sur le bout parabolique. Comme $p^{*} \mathrm{~d} \theta=\mathrm{d} x$, on calcule explicitement en introduisant les coordonnées polaires $u=\rho \mathrm{e}^{i \theta_{2}}$

$$
\mathcal{X}_{\theta}=\mathrm{d} \theta^{\sharp}=\partial_{\theta}+\alpha \partial_{\theta_{2}} .
$$

On vérifie aisément que la métrique $g^{\mathbb{H}^{2}}+g^{\mathrm{FS}}$ est invariante suivant $\partial_{x}$ et on en déduit que $\hat{g}$ est invariante suivant $\mathcal{X}_{\theta}$ :

$$
\mathcal{L}_{\mathcal{X}_{\theta}} \hat{g}=0 \text {. }
$$

Nous introduisons maintenant sur une variété à bouts paraboliques, une classe de métriques asymptotiques au modèle local $\hat{g}$ (au sens $C^{2}$ ). Dans le cas où $X=\mathbb{P}(\mathcal{E})_{\mid \Sigma}$ est une surface réglée obtenue à partir d'un fibré parabolique, on choisira toujours un modèle local $\hat{g}$ adapté à la structure parabolique.

DÉFInition 3. - Si une variété $X$ possède des bouts paraboliques, on en déduit un modèle local de métrique que nous noterons $\hat{g}$. Nous appellerons métrique « asymptotique au modèle local», toute métrique g sur $X$ telle que $g=$ $\hat{g}+o(\hat{g})$, où $o(\hat{g})$ tend uniformément vers 0 en norme $C^{2}$ lorsqu'on s'approche de l'infini sur bout parabolique.

C'est une condition technique raisonnable qui va nous permettre de démontrer au $\S 4.2$ le théorème suivant, dont on déduit immédiatement le théorème $\mathrm{A}$.

BULletin DE LA SOCIÉtÉ MATHÉMATIQUE DE FRANCE 
ThÉORÈme 2.1. - Soit $\mathcal{E} \longrightarrow \bar{\Sigma}$ un fibré parabolique holomorphe de rang 2 sur une surface de Riemann compacte $\bar{\Sigma}$. Soit $\left(P_{i}\right)_{1 \leq i \leq k}$ l'ensemble des points paraboliques de $\bar{\Sigma}$. Nous supposons en outre $\Sigma=\bar{\Sigma} \backslash\left\{P_{i}\right\}$ hyperbolique. Si la surface complexe réglée associée $M=\mathbb{P}(\mathcal{E})_{\Sigma}$ admet une métrique kählérienne $g^{\mathrm{K}}$ à courbure scalaire constante $s=2(c-1) \leq 0$ asymptotique au modèle local $\hat{g}$, alors $\mathcal{E}$ est paraboliquement polystable. De plus une telle métrique provient à un biholomorphisme près, du modèle $\left(\Sigma \times_{\rho} \mathbb{C P}^{1}, \hat{g}\right)$ où $\rho: \pi_{1}(\Sigma) \longrightarrow$ $\mathrm{PU}(2)$ est une représentation associée au fibré parabolique polystable $\mathcal{E}$.

2.3.2. Compactification du bout parabolique rationnel. - Nous faisons maintenant l'hypothèse $\alpha_{2}-\alpha_{1}=r / q \in \mathbb{Q}$, où $r$ et $q$ sont premiers entre eux. Dans ce cas, l'action de $\tau$ définie par (10) vérifie

$$
\tau^{q} \cdot(\xi,[\tilde{u}: \tilde{v}])=(\xi+2 \pi q,[\tilde{u}: \tilde{v}])
$$

On en déduit que $\left(I_{a} \times \mathbb{C P}^{1}\right) /\left\langle\tau^{q}\right\rangle \simeq\left(I_{a} /\left\langle\tau^{q}\right\rangle\right) \times \mathbb{C P}^{1}$. On vient donc de montrer que le revêtement $p: I_{a} \times \mathbb{C P}^{1} \longrightarrow \mathcal{B}$ se factorise par un revêtement $p^{q}$ à $q$ feuillets dont le groupe d'automorphismes est égal à $\langle\tau\rangle /\left\langle\tau^{q}\right\rangle \simeq \mathbb{Z}_{q}$. On a donc un diagramme commutatif holomorphe

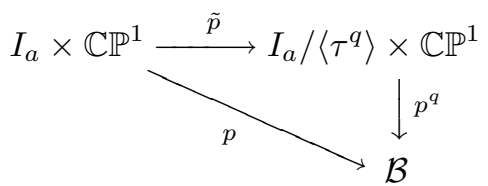

où $\tilde{p}$ désigne la projection canonique sur le revêtement quotient. On note

$$
\begin{aligned}
\pi^{q}: \Delta_{a / q} \simeq I_{a} /\left\langle\tau^{q}\right\rangle \cup\{\infty\} & \longrightarrow \Delta_{a} \simeq I_{a} /\langle\tau\rangle \cup\{\infty\}, \\
z & \longmapsto z^{q}
\end{aligned}
$$

le revêtement holomorphe ramifié à $q$ feuillets du disque par lui-même. Le groupe d'automorphisme $\mathbb{Z}_{q} \simeq\langle\tau\rangle /\left\langle\tau^{q}\right\rangle$ du revêtement $\pi^{q}$ agit par $\tau \cdot \xi=\xi+2 \pi$ soit $\tau \cdot z=\exp (2 i \pi / q) z$. On déduit de $\pi^{q}$ une projection holomorphe notée également

$$
\pi^{q}: I_{a} /\left\langle\tau^{q}\right\rangle \cup\{\infty\} \times \mathbb{C P}^{1} \longrightarrow \Delta_{a}
$$

Par construction, il est clair que $\pi \circ p=\pi_{1}=\pi^{q} \circ \tilde{p}$; on en déduit le lemme :

LEMme 1 (structure du bout parabolique rationnel). - Supposons que $\alpha=$ $\alpha_{2}-\alpha_{1}=r / q \in \mathbb{Q}$, avec $r$ et $q$ premiers entre eux. Le revêtement standard $p: I_{a} \times \mathbb{C P}^{1} \rightarrow \mathcal{B}$ du bout parabolique $\mathcal{B}_{a}^{\alpha_{1}, \alpha_{2}}$ se factorise par un revêtement $\grave{a}$

TOME $130-2002-\mathrm{N}^{\mathrm{O}} 3$ 
$q$ - feuillets $p^{q}$ et on a le diagramme holomorphe commutatif

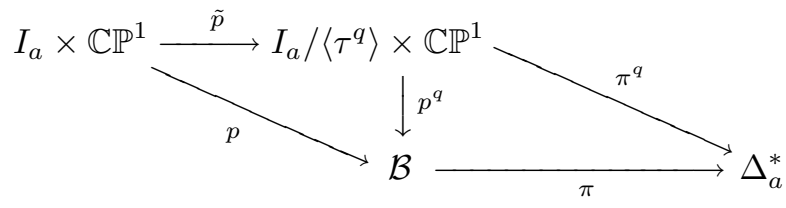

L'action de $\mathbb{Z}_{q}$ sur $I_{a} /\left\langle\tau^{q}\right\rangle \times \mathbb{C P}^{1} \simeq \Delta_{a / q}^{*} \times \mathbb{C P}^{1}$ s'étend clairement en une action holomorphe sur $\Delta_{a / q} \times \mathbb{C P}^{1}$ : pour le voir, il suffit de l'écrire dans des coordonnées adaptées $(z,[\tilde{u}: \tilde{v}])$

$$
\tau \cdot(z,[\tilde{u}: \tilde{v}])=\left(z \mathrm{e}^{\frac{2 i \pi}{q}},\left[\tilde{u} \mathrm{e}^{2 i \pi \alpha_{1}}: \tilde{v} \mathrm{e}^{2 i \pi \alpha_{2}}\right]\right) .
$$

Alors, comme nous l'avons déjà remarqué, $\pi^{q}$ se prolonge de façon holomorphe en $\pi^{q}: \Delta_{a / q} \times \mathbb{C P}^{1} \rightarrow \Delta_{a}$; au quotient, on obtient un orbifibré holomorphe en droites projectives

$$
\overline{\mathcal{B}}=\left(\Delta_{a / q} \times \mathbb{C P}^{1}\right) / \mathbb{Z}_{q}=\mathcal{B} \cup\left(\mathbb{C P}^{1} / \mathbb{Z}_{q}\right) \stackrel{\pi}{\longrightarrow} \Delta_{a} .
$$

Reformulons cette discussion dans le corollaire suivant.

Corollaire 1. - Si $\alpha=\alpha_{2}-\alpha_{1}=r / q \in \mathbb{Q}$ avec $r$ et $q$ premiers entre eux, le $\mathbb{C P}^{1}$-fibré $\mathcal{B} \rightarrow \Delta_{a}^{*}$ admet un prolongement holomorphe en un orbifibré

$$
\overline{\mathcal{B}}=\mathcal{B} \cup\left(\mathbb{C P}^{1} / \mathbb{Z}_{q}\right) \stackrel{\pi}{\longrightarrow} \Delta_{a} .
$$

Cet orbifibré possède un revêtement ramifié à $q$ feuillets $p^{q}: \Delta_{a / q} \times \mathbb{C P}^{1} \rightarrow \overline{\mathcal{B}}$ tel que $\left(p^{q}\right)^{*} \hat{g}$ est égale à la métrique produit standard sur $\Delta_{a / q}^{*} \times \mathbb{C P}^{1}$. La fibre $\pi^{-1}(0) \simeq \mathbb{C P}^{1} / \mathbb{Z}_{q}$ sera appelée « diviseur à l'infini du bout parabolique ».

En conclusion, une variété $M$ avec des bouts paraboliques vérifiant la condition de rationalité admet une compactification orbifold $\bar{M}$. Si $M$ est de plus une variété complexe, la compactification est holomorphe.

\section{Cohomologie $L^{2}$.}

Dans toute cette partie $M$ est une variété $M$ munie de bouts paraboliques et on étudie la cohomologie $\mathrm{H}_{L^{2}}^{*}(M)$ définie à l'aide du complexe des formes différentielles $L^{2}(\hat{g})$ sur $M$. Notons que pour une métrique $g$ asymptotique au modèle local $\hat{g}$ les formes différentielles $L^{2}$ relativement à $g$ et $\hat{g}$ coïncident ainsi donc que les espaces de cohomologies $L^{2}$ associés.

Nous allons démontrer un lemme de Poincaré « à l'infini » sur le bout parabolique de $M$ pour la cohomologie $L^{2}$, afin de la calculer dans le cas des surfaces réglées.

Lorsque les poids des bouts paraboliques vérifient la condition $\alpha_{2}-\alpha_{1} \in \mathbb{Q}$, nous définirons au $\S 3.1$ sur la compactification orbifold $\bar{M}$ des métriques

BULLETIN DE LA SOCiÉtÉ MATHÉMATIQUE DE FRANCE 
lisses $g_{j}$ qui approximent la métrique singulière $g$. On développera alors la théorie de Hodge en montrant des résultats de convergence sur tout compact de $M$ des représentants $g_{j}$ harmoniques d'une classe de cohomologie vers le représentant $g$-harmonique $L^{2}$ ( $c f$. prop. 3.2 et 3.4 ).

3.1. Approximation par des métriques lisses. - Dans $\S 3.1$ nous supposons que les bout paraboliques de $M$ vérifient la condition de rationalité et nous expliquons comment approximer la métrique $g$, singulière sur $\bar{M}$, par une suite de métriques $\left(g_{j}\right)$ lisses sur $\bar{M}$.

3.1.1. Approximation du modèle local $\hat{g}$. — D'après le lemme 1 et le corollaire 1, tout bout parabolique compactifié de $\bar{M}$ admet un revêtement ramifié holomorphe à $q$ feuillets $p^{q}: \Delta_{a / q} \times \mathbb{C P}^{1} \rightarrow \overline{\mathcal{B}}$, tel que $\left(p^{q}\right)^{*} \hat{g}$ est la métrique standard $g^{\Delta^{*}} \oplus g^{\mathrm{FS}}$. On va approximer $\hat{g}$ en remplaçant la métrique singulière $g^{\Delta^{*}}$ par une métrique lisse sur $\Delta$, invariante sous l'action de $\mathbb{Z}_{q}$. Puisque

$$
g^{\Delta^{*}}=\frac{|\mathrm{d} z|^{2}}{(|z| \cdot \ln |z|)^{2}}
$$

on peut par exemple multiplier cette métrique par un facteur conforme singulier de la forme $\lambda(|z|)$, valant $(|z| \ln |z|)^{2}$ au voisinage de l'origine et 1 loin de l'origine. Alors la métrique kählérienne $g_{\lambda}=\lambda(|z|) g^{\Delta^{*}}$ est lisse sur $\Delta$; cette opération consiste simplement à recoller un disque plat au bout du cusp et $g_{\lambda}$ est $\mathbb{Z}_{q}$-invariante car la fonction $\lambda(|z|)$ est elle même invariante. Alors la métrique $g_{\lambda} \oplus g^{\mathrm{FS}}$ passe au quotient et définit une métrique kählérienne lisse sur $\overline{\mathcal{B}}$. Si on suit les géodésiques de $g_{\lambda}$ normales à un cercle, on en déduit une nouvelle coordonnée locale $t^{\prime}$ telle que $t=t^{\prime}$ là où $\lambda(|z|)=1$ et $g_{\lambda}=\mathrm{d} t^{\prime 2}+\varphi\left(t^{\prime}\right)^{2} \mathrm{~d} \theta^{2}$. De façon équivalente, on peut donc définir ce type de métrique en se donnant la fonction $\varphi\left(t^{\prime}\right)$.

Soit la métrique

$$
g_{j}^{\Delta}=\mathrm{d} t^{2}+\varphi_{j}^{2} \mathrm{~d} \theta^{2}
$$

où $\varphi_{j}$ est définie comme suit : pour $t \leq j+1$, on pose $\varphi_{j}=\mathrm{e}^{-t}$. Puis pour $j+1 \leq t \leq j+1+\delta_{j}$, on fait brutalement décroître $\varphi_{j}^{\prime \prime}$ de $\mathrm{e}^{-(j+1)}$ jusqu'à 0 pour un $\delta_{j}$ que nous ferons par la suite tendre vers 0 . Enfin pour $j+1+\delta_{j} \leq$ $t \leq j+1+\epsilon$, on peut quitte à rendre $\varphi_{j}^{\prime \prime}$ très négative faire passer $\varphi_{j}^{\prime}$ dans cet intervalle de $-e^{-(j+1)}$ à -1 .

On a recollé la métrique la métrique de cusp (pour $t \leq j+1$ ) à la métrique plate. En effet si $\varphi_{j}^{\prime}=-1$, alors pour un $T_{j}$ légèrement supérieur à $j+1+\epsilon$ bien choisit, on a $\varphi_{j}=T_{j}-t$. Comme le montre le changement de variable $t^{\prime}=T_{j}-t$, la métrique $g_{j}^{\Delta}$ se recolle à la métrique plate sur le disque; il en résulte que $g_{j}^{\Delta}$ est en réalité lisse sur le disque $\Delta_{a / q}$ tout entier. On vérifie que quitte à choisir $\delta_{j}$ suffisamment petit, on peut supposer que le rapport $-\varphi_{j}^{\prime} / \varphi_{j}$ est croissant, et reste donc minoré par 1. Nous résumons cette construction

TOME $130-2002-\mathrm{N}^{\mathrm{O}} 3$ 
dans le dessin ci-dessous.

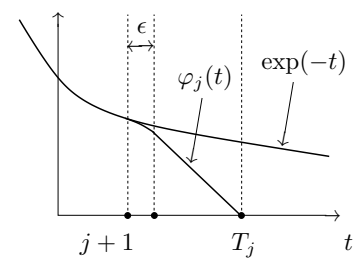

Finalement la métrique $g_{j}^{\Delta} \oplus g^{\mathrm{FS}}$ est lisse et $\mathbb{Z}_{q}$-invariante sur $\Delta_{a / q} \times \mathbb{C P}^{1}$. On en déduit une métrique $\hat{g}_{j}$ lisse sur $\overline{\mathcal{B}}$ qui s'écrit dans les coordonnées $(t, \theta, u)$

$$
\hat{g}_{j}=\mathrm{d} t^{2}+\varphi_{j}^{2} \mathrm{~d} \theta^{2}+\frac{4 / c}{\left(1+|u|^{2}\right)^{2}}|\mathrm{~d} u-i \alpha u \mathrm{~d} \theta|^{2} .
$$

3.1.2. Approximation d'une métrique asymptotique au modèle. - Étant donnée une métrique riemannienne $g$ sur $M$, asymptotique au modèle local sur le bout parabolique de $M$, on approxime la métrique $g$ par des métriques $g_{j}$ lisses sur la compactification $\bar{M}$ comme suit : soit $\chi(t)$ une fonction lisse telle que

- $\chi$ est croissante,

- $\chi(t)=0$ pour $t \leq 0$,

- $\chi(t)=1$ pour $t \geq \frac{1}{2}$.

Notons $\chi_{j}(t)=\chi(t-j)$, puis posons

$$
g_{j}=\left(1-\chi_{j}\right) g+\chi_{j} \hat{g}_{j} .
$$

Alors puisque $g$ est asymptotique au modèle local, les métriques $g_{j}$ et $\hat{g}_{j}$ sont également très proche au sens $C^{2}$ sur la zone de recollement $j \leq t \leq j+1$, et on en déduit en particulier que

$$
\sup _{t \geq j}\left\{\left|\hat{g}_{j}-g_{j}\right|+\left|\nabla^{\hat{g}_{j}}-\nabla^{g_{j}}\right|+\left|R^{\hat{g}_{j}}-R^{g_{j}}\right|\right\} \longrightarrow 0 \text { lorsque } j \rightarrow \infty .
$$

Le modèle local de métrique $\hat{g}$ est à courbure bornée puisque c'est un produit local modelé sur $\mathbb{H}^{2} \times \mathbb{C P}^{1}$. Donc toute métrique asymptotique au modèle local est à courbure bornée.

Lorsqu'on approxime la métrique $\hat{g}$ par des métrique $\hat{g}_{j}$ lisses sur la compactification $\bar{M}$, on crée une «bulle » de courbure très positive. Pour calculer la courbure de $\hat{g}_{j}$, il nous suffit de calculer celle du facteur modifié :

$$
g_{j}^{\Delta}=\mathrm{d} t^{2}+\varphi_{j}^{2} \mathrm{~d} \theta^{2}
$$

La connexion associée est explicite et on a :

$$
\begin{array}{ll}
\nabla_{\partial_{t}} \partial_{t}=0, & \nabla_{\partial_{t}}\left(\varphi_{j}^{-1} \partial_{\theta}\right)=0 \\
\nabla_{\left(\varphi_{j}^{-1} \partial_{\theta}\right)} \partial_{t}=\frac{\partial_{t} \varphi_{j}}{\varphi_{j}} \varphi_{j}^{-1} \partial_{\theta}, & \nabla_{\left(\varphi_{j}^{-1} \partial_{\theta}\right)} \varphi_{j}^{-1} \partial_{\theta}=-\frac{\partial_{t} \varphi_{j}}{\varphi_{j}} \partial_{t}
\end{array}
$$

BULLETIN DE LA SOCIÉTÉ MATHÉMATIQUe DE FRANCE 
on en déduit que la courbure sectionnelle de $g_{j}^{\Delta}$ est égale à

$$
K^{g_{j}^{\Delta}}\left(\partial_{t}, \varphi_{j}^{-1} \partial_{\theta}\right)=-\frac{\partial_{t}^{2} \varphi_{j}}{\varphi_{j}} .
$$

Cette courbure par définition de $\varphi_{j}$ supérieure à -1 , très positive sur l'anneau $t \in[j+1, j+1+\epsilon]$ et uniformément bornée en dehors. Il en résulte que la courbure scalaire de $\hat{g}_{j}=p_{*}^{q}\left(g_{j}^{\Delta}+g^{\mathrm{FS}}\right)$ est donnée par

$$
s^{\hat{g}_{j}}=2\left(c-\frac{\partial_{t}^{2} \varphi_{j}}{\varphi_{j}}\right),
$$

et que $s_{b}^{\hat{g}_{j}}:=s^{\hat{g}_{j}}+2 \chi_{j} \partial_{t}^{2} \varphi_{j} / \varphi_{j}$ est uniformément borné. Puisque la métrique $g$ est asymptotique au modèle local $\hat{g}$, on en déduit d'après (16) un résultat analogue pour les métriques $g_{j}$ :

LEMME 2. - La courbure scalaire de la métrique $g_{j}$ s'écrit

$$
s^{g_{j}}=s_{b}^{g_{j}}-2 \chi_{j} \frac{\partial_{t}^{2} \varphi_{j}}{\varphi_{j}},
$$

où $s_{b}^{g_{j}}$ est uniformément borné.

Par construction des métrique $\hat{g}_{j}$, nous avons contracté le volume initial de $\hat{g}$. On en déduit le lemme technique suivant :

LEMME 3 (contrôle du volume). - Quel que soit $\epsilon>0$, il existe un compact $K$ de $M$, tel que $\mathrm{vol}^{g_{j}}(M \backslash K) \leq \epsilon$ et $\mathrm{vol}^{g}(M \backslash K) \leq \epsilon$. Le volume des métriques $g_{j}$ est donc uniformément borné.

3.2. Lemme de Poincaré à l'infini. - Dans tout le $\S 3.2$, on ne suppose plus en général que la condition de rationalité des poids est vérifiée. Nous commençons par démontrer des estimées sur les fonctions ne dépendant que de la courbure moyenne des métriques.

3.2.1. Courbure moyenne. - La courbure moyenne d'une hypersurface est définie en général comme la trace normalisée de la seconde forme fondamentale. Avec nos notations, on la définit de la façon suivante :

DÉFInITION 4. - Soitg une métrique riemannienne sur $\left[t_{1}, t_{2}\right] \times N$ de la forme $g=\mathrm{d} t^{2}+g_{t}$ où $g_{t}$ est une métrique sur $N$ dépendant de $t$. On définit la courbure moyenne de la métrique $g$ comme la fonction

$$
H=\frac{1}{3} \operatorname{tr}_{g} \mathbb{I}=-\frac{1}{3} \frac{\partial_{t} \mathrm{vol}^{g_{t}}}{\mathrm{vol}^{g_{t}}}
$$

où II désigne la deuxième forme fondamentale de la tranche $N$ et où vol ${ }^{g_{t}}$ est la forme volume associée à la métrique $g_{t}$. Par commodité, on définit également la fonction $h=\frac{3}{2} H$.

TOME $130-2002-\mathrm{N}^{\mathrm{O}} 3$ 
D'après (9) et (14) les métriques $\hat{g}$ et $\hat{g}_{j}$ sont des produits tordus vérifiant respectivement $h=\frac{1}{2}$ et

$$
h=-\frac{1}{2} \varphi_{j}^{-1} \partial_{t} \varphi_{j},
$$

qui est par construction de $\varphi_{j}$ minoré par $\frac{1}{2}$. Près de $t=T_{j}$, on a $\varphi_{j}=T_{j}-t$ et $h=\frac{1}{2}\left(T_{j}-t\right)^{-1}$ donc $h$ tend vers $+\infty$ quand $t \rightarrow T_{j}$.

Dès que l'on a un contrôle du type $h \geq h_{0}>0$, nous obtenons le lemme suivant qui s'applique en particulier aux métriques $\hat{g}$ et $\hat{g}_{j}$ :

LEMme 4. - Soit $g=\mathrm{d} t^{2}+g_{t}$ une métrique riemannienne sur $\left[t_{1}, t_{2}\right] \times N$. Supposons qu'il existe $\delta, h_{0} \in \mathbb{R}$ telles que g vérifie $h \geq h_{0}>\delta$ ou $\delta>h_{0} \geq h$. Alors pour toute fonction $f$, on a

$$
\text { (18) } \begin{aligned}
\int_{\left[t_{1}, t_{2}\right] \times N}\left|\mathrm{e}^{\delta t} \partial_{t} f\right|^{2} \mathrm{vol}^{g} & \geq\left(h_{0}-\delta\right)^{2} \int_{\left[t_{1}, t_{2}\right] \times N}\left|f \mathrm{e}^{\delta t}\right|^{2} \mathrm{vol}^{g} \\
& +\left(h_{0}-\delta\right)\left\{\int_{t=t_{2}}\left|f \mathrm{e}^{\delta t}\right|^{2} \mathrm{vol}^{g}-\int_{t=t_{1}}\left|f \mathrm{e}^{\delta t}\right|^{2} \mathrm{vol}^{g}\right\} .
\end{aligned}
$$

Démonstration. - Voir [3], lemme 4.1, pour la démonstration dans le cas $h>h_{0}>0$. En général, la démontration est identique en intégrant $f \mathrm{e}^{\delta t}$ par parties cette fois-ci.

3.2.2. Identification de la cohomologie $L^{2}$. - Le $\S 3.2 .1$ fournit des outils suffisants pour obtenir le lemme de Poincaré à l'infini pour la cohomologie $L^{2}$ suivant :

Lemme 5 (lemme de Poincaré $L^{2}$ à l'infini). - Soit $\gamma$, une $k$-forme fermée $L^{2}$ sur la variété à bouts paraboliques $M$.

- Pour $k=1,3$ ou 4, il existe une $(k-1)$-forme $\beta, L^{2}$ définie sur le bout parabolique de $M$ telle que $\gamma=\mathrm{d} \beta$ sur le bout parabolique.

- Dans le cas où $k=2$, il existe $\lambda \in \mathbb{R}$ et une 1 -forme $\beta L^{2}$ définie sur le bout parabolique de $M$ tels que $\gamma=\lambda \mathrm{vol}^{\mathbb{C P}^{1}}+\mathrm{d} \beta$ sur le bout parabolique.

Rappelons que vol ${ }^{\mathbb{C P}^{1}}$ est la 2-forme fermée induite sur les bouts parabolique par la métrique de Fubini-Study (cf. 2.3.1). Admettons pour l'instant ce lemme qui va nous permettre de calculer la cohomologie $L^{2}$ de $M$.

Dans le cas où $\alpha_{2}-\alpha_{1} \in \mathbb{Q}$, on dispose également d'un lemme de Poincaré analogue pour la cohomologie de de Rham orbifold sur le bout parabolique de $\bar{M}$. Rappelons qu'une forme différentielle lisse au sens orbifold sur le bout parabolique compactifié $\overline{\mathcal{B}}$ de $\bar{M}$ est donnée par une forme différentielle lisse et $\mathbb{Z}_{q}$-invariante sur le revêtement ramifié. On remarque alors que si $\gamma=\mathrm{d} \beta$ est $\mathbb{Z}_{q}$-invariante sur $\Delta_{a} \times \mathbb{C P}^{1}$ avec $\beta$ a priori non invariante, alors il existe une forme $\mathbb{Z}_{q}$-invariante $\beta^{\prime}$ obtenue en moyennant $\beta$ sous l'action du groupe, telle 
que $\gamma=\mathrm{d} \beta^{\prime}$. On en déduit le lemme de Poincaré suivant pour la cohomologie de De Rham de l'orbifold $\bar{M}$ :

LEMME 6. - Soit $\gamma$, une $k$-forme lisse fermée sur $\bar{M}$.

- Pour $k=1,3$ ou 4, il existe une $(k-1)$-forme lisse $\beta$ telle que $\gamma=d \beta$ sur le bout parabolique de $\bar{M}$.

- Dans le cas où $k=2$, il existe un réel $\lambda$ et une 1 -forme lisse $\beta$ sur $\bar{M}$ tels que $\gamma=\lambda \mathrm{vol}^{\mathbb{C} \mathbb{P}^{1}}+\mathrm{d} \beta$ sur le bout parabolique.

Soit $\bar{M}^{\prime}$ la compactification de $M$ obtenue en changeant la valeurs des poids $\alpha_{1}\left(P_{i}\right), \alpha_{2}\left(P_{i}\right)$ de la structure parabolique en $\alpha_{1}^{\prime}\left(P_{i}\right)$ et $\alpha_{2}^{\prime}\left(P_{i}\right)$ tels que $\alpha^{\prime}\left(P_{i}\right)=\alpha_{1}^{\prime}\left(P_{i}\right)-\alpha_{2}^{\prime}\left(P_{i}\right) \in \mathbb{Q}$. En utilisant le lemme de Poincaré local, on déduit le corollaire suivant.

Corollaire 2. - On a un isomorphisme entre les espaces de cohomologie

$$
\mathrm{H}_{L^{2}(g)}^{*}(M) \simeq \mathrm{H}_{\mathrm{DR}}^{*}\left(\bar{M}^{\prime}\right) .
$$

Démonstration. - Définissons un isomorphisme préservant le cup-produit $F$ : $\mathrm{H}_{L^{2}}^{*}(M) \rightarrow \mathrm{H}_{\mathrm{DR}}^{*}\left(\bar{M}^{\prime}\right)$ comme suit : soit $b \in \mathrm{H}_{L^{2}}^{k}(M)$. Pour $k=0$, on a clairement $\mathrm{H}_{L^{2}}^{0}(M) \simeq \mathbb{R}$ puisque la métrique est de volume fini. Par ailleurs $\mathrm{H}_{\mathrm{DR}}^{0}(M) \simeq \mathbb{R}$ et on définit $F$ par cet isomorphisme. Si $k=1,3$ ou 4 , on peut d'après le lemme de Poincaré 5 , choisir un représentant $\gamma$ à support compact dans $M$ de $b$ et on pose $F(b)=\left[\gamma^{\prime}\right]$.

Pour $k=2$, on peut choisir un représentant $\gamma$ de $b$ tel que $\gamma=\lambda$ vol $^{\mathbb{C P}^{1}}$ sur le bout parabolique en utilisant le lemme de Poincaré. La forme vol ${ }^{\mathbb{C P}^{1}}$ n'est pas a priori lisse sur $\bar{M}^{\prime}$. Notons $\mathrm{vol}^{S^{2}}$ la forme lisse associée à $\bar{M}^{\prime}$. En intégrant sur une fibre où où ces formes sont toutes deux définies, on a par construction $\int_{\mathbb{C P}^{1}} \operatorname{vol}^{\mathbb{C P}^{1}}=\int_{\mathbb{C P}^{1}} \operatorname{vol}^{S^{2}}$, donc vol $\mathbb{C P}^{1}=\operatorname{vol}^{S^{2}}+d \beta_{2}$, où $\beta_{2}$ est une 1-forme lisse sur $\mathcal{B}^{\prime}$ sans aucune hypothèse de régularité à l'infini. Néanmoins, en utilisant une fonction $\chi_{j}(t)$ définie au $\left.\S 3.1 .2\right)$ pour $j$ suffisamment grand, $\gamma^{\prime}=\gamma-\lambda d\left(\chi_{j} \beta\right)$ est lisse sur $\bar{M}^{\prime}$, car elle est égale à $\lambda$ vol ${ }^{S^{2}}$ près de l'infini ; on pose alors $F(b)=\left[\gamma^{\prime}\right]$.

On définit de même la réciproque de $F$ en utilisant cette fois-ci le lemme de Poincaré local pour l'orbifold $\bar{M}^{\prime}$. On en déduit le corollaire.

3.2.3. Cohomologie $L^{2}$ des surfaces réglées. - Soit $M=\mathbb{P}(\mathcal{E})_{\mid \Sigma}$ une surface réglée obtenue à partir d'un fibré parabolique $\mathcal{E} \rightarrow \bar{\Sigma}$ munie d'un modèle local de métrique adapté $\hat{g}$. On considère maintenant la compactification $\widehat{M}=\mathbb{P}(\mathcal{E})_{\mid \bar{\Sigma}}$.

Pour un choix de poids $\alpha_{1}^{\prime}\left(P_{i}\right)=\alpha_{2}^{\prime}\left(P_{i}\right)=0$, on a $\widehat{M}=\bar{M}^{\prime}$, d'où par le corollaire 2 un isomorphisme

$$
\mathrm{H}_{\mathrm{DR}}^{*}(\widehat{M}) \simeq \mathrm{H}_{L^{2}}^{*}(M) .
$$

TOME $130-2002-\mathrm{N}^{\mathrm{O}} 3$ 
Or la cohomologie de la surface réglée $\widehat{M}$ est bien connue (cf. [1], prop. III.18); en particulier la 2-cohomologie vérifie :

$$
H^{2}(\widehat{M}, \mathbb{Z})=\mathbb{Z} h \oplus \mathbb{Z} F,
$$

où $F$ est le dual de Poincaré d'une fibre, et $h$ la classe du fibré tautologique $\mathcal{O}_{\widehat{M}}(1) \operatorname{sur} \widehat{M}$. Alors $h^{2}=\operatorname{deg} \mathcal{E}, F^{2}=0$ et $h \cdot F=1$; on en déduit via l'isomorphisme (19) la proposition suivante :

Proposition 3.1. - On a des isomorphismes

$$
\mathrm{H}_{L^{2}}^{0}(M) \simeq \mathrm{H}_{L^{2}}^{4}(M) \simeq \mathbb{R}, \quad \mathrm{H}_{L^{2}}^{1}(M) \simeq \mathrm{H}_{L^{2}}^{3}(M) \simeq H^{1}(\bar{\Sigma}, \mathbb{R}) .
$$

Il existe des classes de cohomologie $F, h \in \mathrm{H}_{L^{2}}^{2}(M)$ telles que

$$
F^{2}=0, \quad h^{2}=\operatorname{deg} \mathcal{E}, \quad h \cdot F=1 \quad \text { et } \quad \mathrm{H}_{L^{2}}^{2}(M)=\mathbb{R} h \oplus \mathbb{R} F .
$$

En conséquence $b_{2}=2$ et la forme d'intersection est de signature $(1,1)$.

\subsubsection{Démonstration du lemme de Poincaré 5}

Cas des 1-formes. - Sur le bout parabolique, $\gamma$ se décompose en $\gamma=f \mathrm{~d} t+\gamma_{2}$, où $\gamma_{2}$ est une 1 -forme telle que $i_{\partial_{t}} \gamma_{2}=0$. Dans le cas où $f$ est à support compact, définissons une «primitive» de $f$ par $\tilde{f}=\int_{0}^{t} f \mathrm{~d} t$ nulle en $t=0$. D'après le lemme 4 ,

$$
\int_{t=0}^{T}|f|^{2} \operatorname{vol}^{\hat{g}}=\int_{t=0}^{T}\left|\partial_{t} \tilde{f}\right|^{2} \operatorname{vol}^{\hat{g}} \geq h_{0}^{2} \int_{t=0}^{T}|\tilde{f}|^{2} \mathrm{vol}^{\hat{g}},
$$

car le seul terme de bord non nul est positif. On déduit de cette dernière inégalité que pour $f \in L^{2}$, la fonction $\tilde{f}$ est également définie dans $L^{2}$. Or $\gamma-\mathrm{d} \tilde{f}$ vérifie par définition de $\tilde{f}$

$$
\mathcal{L}_{\partial_{t}}(\gamma-\mathrm{d} \tilde{f})=\left(d \circ i_{\partial_{t}}+i_{\partial_{t}} \circ \mathrm{d}\right)(\gamma-\mathrm{d} \tilde{f})=0 .
$$

Par conséquent $(\gamma-\mathrm{d} \tilde{f})$ est indépendante de $t$, et représente une classe de cohomologie de $\mathrm{H}_{\mathrm{DR}}^{1}\left(S^{1} \times \mathbb{C P}^{1}\right)=\mathbb{R}[d \theta]$; il existe donc une fonction $f_{2}$ indépendante de $t$ (donc $L^{2}$ ), telle que $\gamma-\mathrm{d} \tilde{f}-\mathrm{d} f_{2}=\mu \mathrm{d} \theta$, avec $\mu$ constante.

Soit $\mathcal{X}_{\theta}$ le champ de vecteurs défini par (12). Posons

$$
V(t)=\int_{\{t\} \times N}\left(i_{\mathrm{e}^{t} \mathcal{X}_{\theta}} \gamma\right) \mathrm{vol}^{\hat{g}_{t}} .
$$

Comme $\left|\mathrm{e}^{t} \mathcal{X}_{\theta}\right|=\left|\mathrm{e}^{-t} d \theta\right|=1$ et $\gamma, \operatorname{vol}^{\hat{g}} \in L^{2}$, on en déduit que $\left(i_{\mathrm{e}^{t} \mathcal{X}_{\theta}} \gamma\right) \operatorname{vol}^{\hat{g}}$ est intégrable et on peut donc choisir des tranches $t_{k} \rightarrow \infty$ telles que $V\left(t_{k}\right) \rightarrow 0$. 
Par ailleurs

$$
\begin{aligned}
& \int_{N}\left(i_{\mathrm{e}^{t} \mathcal{X}_{\theta}} \mathrm{d}\left(\tilde{f}+f_{2}\right)\right) \operatorname{vol}^{\hat{g}_{t}}=\int_{N}\left(\mathcal{L}_{\mathrm{e}^{t} \mathcal{X}_{\theta}}\left(\tilde{f}+f_{2}\right)\right) \operatorname{vol}^{\hat{g}_{t}} \\
& =\int_{N} \mathcal{L}_{\mathrm{e}^{t} \mathcal{X}_{\theta}}\left(\left(\tilde{f}+f_{2}\right) \operatorname{vol}^{\hat{g}_{t}}\right)-\int_{N}\left(\tilde{f}+f_{2}\right) \mathcal{L}_{e^{t} \mathcal{X}_{\theta}} \operatorname{vol}^{\hat{g}_{t}} \\
& =\int_{N} \mathrm{~d} \circ i_{\mathrm{e}^{t} \mathcal{X}_{\theta}}\left(\left(\tilde{f}+f_{2}\right) \operatorname{vol}^{\hat{g}_{t}}\right)-\int_{N}\left(\tilde{f}+f_{2}\right) \mathcal{L}_{\mathrm{e}^{t} \mathcal{X}_{\theta}} \operatorname{vol}^{\hat{g}_{t}}=0,
\end{aligned}
$$

car la métrique est invariante suivant $\mathcal{X}_{\theta}(c f .(13))$. On en déduit que

$$
V(t)=\int_{N} i_{\mathrm{e}^{t} \mathcal{X}_{\theta}} \mu \mathrm{d} \theta \mathrm{vol}^{\hat{g}_{t}}=\mu \int_{N} \mathrm{e}^{t} \mathrm{vol}^{\hat{g}_{t}} .
$$

Or $\mathrm{e}^{t} \mathrm{vol}^{\hat{g}_{t}}=\mathrm{d} \theta \wedge \mathrm{vol}^{\mathbb{C P}^{1}}$ est indépendante de $t$; puisque $V\left(t_{k}\right) \rightarrow 0$, on a nécessairement $\mu=0$. Finalement, on a démontré que $\gamma=\mathrm{d}\left(\tilde{f}+f_{2}\right)$, où $\tilde{f}$ et $f_{2}$ sont $L^{2}$ ce qui achève la démonstration pour les 1 -formes.

Cas des 2-formes. - Soit $\gamma$ une 2-forme fermée et $L^{2}$. On la décompose en $\gamma=\mathrm{d} t \wedge \beta+\gamma_{2}$, où $\beta$ et $\gamma_{2}$ sont des formes différentielles telles que $i_{\partial_{t}} \gamma_{2}=0$ et $i_{\partial_{t}} \beta=0$. Comme dans le cas des fonctions, nous cherchons une 1 -forme $\tilde{\beta}$ dans $L^{2}$, telle que $i_{\partial_{t}} \tilde{\beta}=0$ et $\mathcal{L}_{\partial_{t}} \tilde{\beta}=\beta$. On a un la décomposition $\beta=\mu \mathrm{d} \theta+\beta_{2}$, où $\mu$ est une fonction, et $\beta_{2}$ une 1 -forme telle que $i_{\partial_{t}} \beta_{2}=i_{\partial_{\theta}} \beta_{2}=0$. Comme $\gamma$ est $L^{2}$ par hypothèse, on en déduit que $\beta_{2}$ et $\mu e^{t}$ sont $L^{2}$. Pour la partie $\beta_{2}$, la technique est semblable au cas des fonctions; on pose $\tilde{\beta}_{2}=\int_{0}^{t} \beta_{2} \mathrm{~d} t$, alors d'après le lemme 4 , toujours en utilisant le fait que le terme de bord est nul en $t=0$, on obtient pour $\beta_{2}$ à support compact

$$
\int_{0}^{\infty}\left|\tilde{\beta}_{2}\right|^{2} \operatorname{vol}^{\hat{g}} \leq \int_{0}^{\infty}\left|\partial_{t} \tilde{\beta}_{2}\right|^{2} \operatorname{vol}=\int_{0}^{\infty}\left|\beta_{2}\right|^{2} \operatorname{vol}^{\hat{g}}
$$

On en déduit que $\tilde{\beta}_{2}$ est définie dans $L^{2}$ alors $\beta_{2} \in L^{2}$.

Dans le cas où $\mu$ est à support compact, on définit $\tilde{\mu}=\int_{t}^{\infty} \mu \mathrm{d} t$; d'après le lemme 4

$$
\int_{0}^{\infty}|\tilde{\mu} \mathrm{d} \theta|^{2} \operatorname{vol}^{\hat{g}}=\int_{0}^{\infty}\left|\tilde{\mu} \mathrm{e}^{t}\right|^{2} \operatorname{vol}^{\hat{g}} \leq \int_{0}^{\infty}\left|\left(\partial_{t} \tilde{\mu}\right) \mathrm{e}^{t}\right|^{2} \operatorname{vol}^{\hat{g}}=\int_{0}^{\infty}|\mu \mathrm{d} \theta|^{2} \operatorname{vol}^{\hat{g}} .
$$

On en déduit que $\tilde{\mu} \mathrm{d} \theta \in L^{2}$ si $\mu \mathrm{d} \theta \in L^{2}$. Posons maintenant $\tilde{\beta}=\tilde{\mu} \mathrm{d} \theta+\tilde{\beta}_{2} \in$ $L^{2}$ et $\gamma_{3}=\gamma-\mathrm{d} \tilde{\beta}$. Alors $i_{\partial_{t}} \gamma_{3}=0$ et $\mathrm{d} \gamma_{3}=0$, d'où $\mathcal{L}_{\partial_{t}} \gamma_{3}=0$, ce qui implique que $\gamma_{3}$ est une 2-forme sur la tranche indépendante de $t$. On écrit alors $\gamma_{3}=\beta_{3} \wedge \mathrm{d} \theta+\gamma_{4}$, avec $\beta_{3}$ et $\gamma_{4}$ des formes sur la tranche, indépendantes de $t$ telles que $i_{\mathcal{X}_{\theta}} \beta_{3}=0$ et $i_{\mathcal{X}_{\theta}} \gamma_{4}=0$.

On dira que $X$ est un champ de vecteurs horizontal sur $\mathcal{B}$ s'il est indépendant de $t$ et si $p^{*} X$ (où $p: I_{a} \times \mathbb{C P}^{1} \rightarrow \mathcal{B}$ est le revêtement universel riemannien associé au bout parabolique) est un champ de vecteurs tangent au facteur $\mathbb{C P}^{1}$ invariant sous l'action de $\tau$.

TOME $130-2002-\mathrm{N}^{\mathrm{O}} 3$ 
Si $X$ est un champ de vecteurs horizontal, $|X|_{\hat{g}}$ et $\mid \mathcal{L}_{X}$ vol $\left.^{\mathbb{C P}^{1}}\right|_{\hat{g}}$ sont uniformément bornés; le volume étant fini, ceci implique que $\mathcal{L}_{X}$ vol $^{\mathbb{C P}^{1}} \in L^{2}$.

Posons

$$
\begin{aligned}
U(t) & =\int_{\{t\} \times N}\left(i_{X} \gamma\right) \wedge \operatorname{vol}^{\mathbb{C P}}, \\
V(t) & =\int_{\{t\} \times N}\left(i_{X} \mathrm{~d} \tilde{\beta}\right) \wedge \operatorname{vol}^{\mathbb{C P}^{1}}=\int_{N} \mathcal{L}_{X} \tilde{\beta} \wedge \operatorname{vol}^{\mathbb{C P}^{1}}=\int_{N} \tilde{\beta} \wedge \mathcal{L}_{X} \operatorname{vol}^{\mathbb{C P}} .
\end{aligned}
$$

Comme $\gamma \in L^{2}$ on a $i_{X} \gamma \in L^{2}$; de plus $\mathcal{L}_{X}$ vol $^{\mathbb{C P}^{1}}$ et $\tilde{\beta}$ sont dans $L^{2}$. On peut donc choisir des tranches $t_{k} \rightarrow \infty$ de sorte que $U\left(t_{k}\right) \rightarrow 0$ et $V\left(t_{k}\right) \rightarrow 0$. Mais

$$
U\left(t_{k}\right)-V\left(t_{k}\right)=\int_{\left\{t_{k}\right\} \times N} i_{X} \gamma_{3} \wedge \mathrm{vol}^{\mathbb{C P}^{1}}=\int_{N}\left(i_{X} \beta_{3}\right) \mathrm{d} \theta \wedge \mathrm{vol}^{\mathbb{C P}^{1}}
$$

est indépendant de $k$. Dès que $\beta_{3} \neq 0$, on peut choisir $X$ tel que cette intégrale soit non nulle. Nécessairement on a alors $\beta_{3}=0$. On en déduit que $\gamma_{3}=\gamma_{4}$ est une 2-forme sur la tranche indépendante de $t$ telle que $i_{\mathcal{X}_{\theta}} \gamma_{3}=0, \mathrm{~d} \gamma_{3}=0$, d'où $\mathcal{L}_{\mathcal{X}_{\theta}} \gamma_{3}=0$. On en déduit en passant au revêtement que $p^{*} \gamma_{3}$ est une 2 -forme $\mathbb{Z}$-invariante sur le facteur $\mathbb{C P}^{1}$.

En utilisant la cohomologie de De Rham usuelle, on peut écrire $\gamma_{3}$ sous la forme $\lambda$ vol $\mathbb{C P}^{1}+\mathrm{d} \beta_{4}$, avec $\beta_{4}$ une 1-forme sur $\mathbb{C P}^{1}$. Si $\alpha_{2}-\alpha_{1} \in \mathbb{Q}$, l'action de $\mathbb{Z}$ induite sur $\mathbb{C P}^{1}$ se factorise en une action de $\mathbb{Z}_{q}$. La 1 -forme $\beta_{4}$ n'est pas nécessairement invariante, mais quitte à moyenner sous l'action de $\mathbb{Z}_{q}$, on peut la supposer invariante de sorte que $\beta_{4}$ passe au quotient et $\beta_{4} \in L^{2}$. On a donc démontré que $\gamma=\lambda \mathrm{vol}^{\mathbb{C P}}+\mathrm{d}\left(\tilde{\beta}+\beta_{4}\right)$ avec $\tilde{\beta}$ et $\beta_{4} \in L^{2}$.

Lorsque $\alpha_{2}-\alpha_{1} \notin \mathbb{Q}$, l'action de $\mathbb{Z}$ sur $\mathbb{C P}^{1}$ ne se factorise pas. Cette action est engendrée par une rotation $\mathcal{R}_{\alpha}$ d'angle $-2 \pi \alpha$ donnée par $\tau \cdot[\tilde{u}: \tilde{v}]=$ $\left[\tilde{u} \mathrm{e}^{2 i \pi \alpha_{1}}: \tilde{v} \mathrm{e}^{2 i \pi \alpha_{2}}\right]$. Comme $\alpha \notin \mathbb{Q}$, le groupe engendré par $\mathcal{R}_{\alpha}$ est dense dans le groupe des rotations de même axe. Par densité, $\gamma_{4}$ est invariante par toute rotation $\mathcal{R}_{\varphi}$ de ce type. Puisque la forme volume vol ${ }^{\mathbb{C P}^{1}}$ est invariante par rotation, la forme exacte $\gamma_{3}-\lambda \mathrm{vol}^{\mathbb{C P}^{1}}$ est également invariante par rotation. On en déduit comme dans le cas rationnel, en moyennant sur l'action de $\mathcal{R}_{\varphi}$ pour $\varphi \in S^{1}$, une 1-forme $\beta_{4}$ invariante par rotation telle que $\gamma_{3}-\lambda$ vol $^{\mathbb{C P}^{1}}=\mathrm{d} \beta_{4}$. En particulier, $\beta_{4}$ est invariante par $\tau$ et passe au quotient.

Cas des 3-formes. - Une 3-forme sur le bout parabolique se décompose en $\Omega=\mathrm{d} t \wedge\left(\beta \wedge \mathrm{d} \theta+\lambda \mathrm{vol}^{\mathbb{C P}^{1}}\right)+\Omega_{2}$, où $\beta$ et $\Omega_{2}$ vérifient $i_{\partial_{t}} \beta=i_{\partial_{\theta}} \beta=0$ et $i_{\partial_{t}} \Omega_{2}=0$. On cherche des «primitives » de $\beta$ et $\lambda$ en posant $\tilde{\beta}=\int_{t}^{\infty} \beta \mathrm{d} t$ et $\tilde{\lambda}=\int_{0}^{t} \lambda \mathrm{d} t$ dans le cas de formes à support compact. D'après le lemme 4 ,

$$
\int_{0}^{\infty}|\tilde{\beta} \wedge \mathrm{d} \theta|^{2} \operatorname{vol}^{\hat{g}} \leq \frac{1}{h_{0}^{2}} \int_{0}^{\infty}\left|\left(\partial_{t} \tilde{\beta}\right) \mathrm{e}^{t}\right|^{2} \operatorname{vol}^{\hat{g}}=\frac{1}{h_{0}^{2}} \int_{0}^{\infty}|\beta \wedge \mathrm{d} \theta|^{2} \operatorname{vol}^{\hat{g}},
$$

BULLETIN DE LA SOCIÉtÉ MATHÉMATIQUE DE FRANCE 


$$
\int_{0}^{\infty}\left|\tilde{\lambda} \operatorname{vol}^{\mathbb{C P}}\right|^{2} \operatorname{vol}^{\hat{g}}=\int_{0}^{\infty}|\tilde{\lambda}|^{2} \operatorname{vol}^{\hat{g}} \leq \frac{1}{h_{0}^{2}} \int_{0}^{\infty}\left|\partial_{t} \tilde{\lambda}\right|^{2} \operatorname{vol}=\frac{1}{h_{0}^{2}} \int_{0}^{\infty}|\lambda|^{2} \operatorname{vol}^{\hat{g}},
$$

ce qui donne un sens à $\tilde{\beta} \wedge \mathrm{d} \theta$ et $\tilde{\lambda}$ dans $L^{2}$ pour $\lambda$ et $\beta \wedge \mathrm{d} \theta \in L^{2}$. On en déduit une 2-forme $L^{2}$ définie par $\omega=\tilde{\beta} \wedge \mathrm{d} \theta+\tilde{\lambda}$ vol $^{\mathbb{C P}^{1}}$, telle que $\Omega_{3}=\Omega-\mathrm{d} \omega$ vérifie $i_{\partial_{t}} \Omega_{3}=0$, par définition de $\omega$. Alors $\mathcal{L}_{\partial_{t}} \Omega_{3}=\left(\mathrm{d} \circ i_{\partial_{t}}+i_{\partial_{t}} \circ \mathrm{d}\right) \Omega_{3}=0$, donc $\Omega_{3}$ est une 3-forme indépendante de $t$ sur la tranche $N \simeq S^{1} \times \mathbb{C P}^{1}$ du bout parabolique. On peut donc l'écrire $\Omega_{3}=\mu \mathrm{d} \theta \wedge \mathrm{vol}^{\mathbb{C P}^{1}}$, où $\mu$ est une fonction indépendante de $t$.

S'il existe une fonction $f$ constante suivant $\partial_{t}$ et $\mathcal{X}_{\theta}$, telle que $\int_{N} f \mu \mathrm{d} \theta \wedge$ $\operatorname{vol}^{\mathbb{C P}^{1}} \neq 0$, on a alors une contradiction : $f,|\mathrm{~d} f|$ sont bornées car $f$ ne dépend que du facteur $\mathbb{C P}^{1}$. On en déduit que $f$ et $\mathrm{d} f$ sont $L^{2}$ puisque nous sommes en volume fini. Maintenant

$$
\int_{\{t\} \times N} f \Omega=\int_{\{t\} \times N} f \Omega_{3}+\int_{\{t\} \times N} f \mathrm{~d} \omega=\int_{\{t\} \times N} f \Omega_{3}-\int_{\{t\} \times N} \mathrm{~d} f \wedge \omega,
$$

et nous pouvons choisir des tranches $t_{k}$ telles que $\int_{t_{k}} f \Omega$ et $\int_{t_{k}} \mathrm{~d} f \wedge \omega$ tendent vers 0. Par ailleurs $\int_{\{t\} \times N} f \Omega_{3}$ est indépendante de $t$ d'où une contradiction. Par conséquent $\mu$ est orthogonale aux fonctions constantes suivant $\mathcal{X}_{\theta}$ ce qui entraîne l'existence de $\tilde{\mu}$ telle que $\mathcal{X}_{\theta} \cdot \tilde{\mu}=\mu$. Comme $\tilde{\mu}$ vol ${ }^{\mathbb{P P}^{1}}$ est bornée, donc $L^{2}$; on en déduit que $\Omega=\mathrm{d}\left(\omega+\tilde{\mu} \mathrm{vol}^{\mathbb{C P}}\right)$ est exacte au sens de la cohomologie $L^{2}$ sur le bout parabolique.

Cas des 4-formes. - Une 4-forme $L^{2}$ sur le bout parabolique s'écrit $\gamma=\mu \mathrm{d} t \wedge$ $\mathrm{d} \theta \wedge \operatorname{vol}^{\mathbb{C P}^{1}}$, avec $\mu \mathrm{d} \theta \in L^{2}$. Nous avons déjà démontré ( $c f$. cas des 2-formes) qu'il existe une fonction $\tilde{\mu}$ définie sur le bout parabolique telle que $\tilde{\mu} \mathrm{d} \theta \in L^{2}$ et $\partial_{t} \tilde{\mu}=\mu$. On en déduit $\mathrm{d} \tilde{\gamma}=\gamma$ avec $\tilde{\gamma}=\tilde{\mu} \mathrm{d} \theta \wedge \operatorname{vol}^{\mathbb{C P}}{ }^{1} \in L^{2}$, d'où le résultat.

3.3. Convergence des formes harmoniques. - Dans tout le $\S 3.3$, on suppose que les poids des bouts paraboliques de $M$ vérifient la condition $\alpha=\alpha_{2}-\alpha_{1} \in \mathbb{Q}$ par souci de clarté. Puis nous reviendrons plus tard sur le cas irrationnel ( $c f . \S 4.4)$ pour lequel on a des résultats analogues.

3.3.1. Inégalités de Poincaré pour les fonctions. — Les lemmes 3 et 4 nous permettent facilement d'obtenir une borne inférieure uniforme sur la première valeur propre du laplacien des métriques $g_{j}$ pour les fonctions ( $c f$. [3], cor. 4.3).

Lemme 7 (inégalité de Poincaré). — Supposons que $M$ possède des bouts paraboliques vérifiant la condition $\alpha=\alpha_{2}-\alpha_{1} \in \mathbb{Q}$. Soit $g$ une métrique asymptotique au modèle local $\hat{g}$ et soit $g_{j}$ la suite d'approximations de $g$ lisses sur $\bar{M}$. Il existe une constante $c>0$, indépendante de $j$, telle que pour toute fonction $f$ sur $\bar{M}$ vérifiant $\int_{M} f \mathrm{vol}^{g_{j}}=0$, on ait

$$
\int_{M}|\mathrm{~d} f|^{2} \operatorname{vol}^{g_{j}} \geq c \int_{M}|f|^{2} \operatorname{vol}^{g_{j}},
$$

TOME $130-2002-\mathrm{N}^{\mathrm{O}} 3$ 
les normes étant prises par rapport à la métrique $g_{j}$.

On en déduit l'inégalité de Poincaré à la limite :

Corollaire 3 (inégalité de Poincaré). — Soit g une métrique asymptotique au modèle local sur $M$. Il existe une constante $c>0$ telle que pour toute fonction $f \in L_{1}^{2}(g)$ vérifiant $\int_{M} f \mathrm{vol}^{g}=0$, on ait

$$
\int_{M}|\mathrm{~d} f|^{2} \mathrm{vol}^{g} \geq c \int_{M}|f|^{2} \mathrm{vol}^{g} .
$$

Démonstration. - On applique le corollaire 7 à $h_{j}=\left(1-\chi_{j}\right)\left(f+c_{j}\right)$, où $c_{j}$ est une constante choisie de sorte que $\int h_{j} \mathrm{vol}^{g_{j}}=0$. On en déduit le résultat en passant à la limite. Comme nous le verrons au $\S 4.4$, cette démonstration, dans laquelle nous avons fait implicitement l'hypothèse $\alpha_{2}-\alpha_{1} \in \mathbb{Q}$, s'étend même si cette condition n'est pas vérifiée.

Remarque. - Cette inégalité de Poincaré permet classiquement de développer la théorie de Hodge des 1-formes $g$-harmoniques $L^{2}$. On en déduit que toute classe de cohomologie de $\mathrm{H}_{L^{2}}^{1}(M)$ admet un unique représentant $g$-harmonique $L^{2}$.

3.3.2. Convergence des 1-formes harmoniques. — L'inégalité de Poincaré du lemme 7 sur les fonctions permet maintenant de démontrer le résultat suivant.

Proposition 3.2. - Soit une classe de cohomologie $b \in \mathrm{H}_{\mathrm{DR}}^{1}(\bar{M})$. La suite $\gamma_{j}$ de ses représentants $g_{j}$-harmoniques sur $\bar{M}$ converge au sens $C^{\infty}$ sur tout compact de $M$ vers le représentant $g$-harmonique $L^{2}$ de $b$.

Plus précisément, il existe une constante $c>0$, telle que si l'on choisit un représentant à support compact $\beta$ de b, et qu'on écrit $\gamma_{j}=\beta+\mathrm{d} f_{j}$, où $f_{j}$ est une fonction sur $\bar{M}$ vérifiant $\int_{M} f_{j} \mathrm{vol}^{g_{j}}=0$, alors $f_{j}$ converge sur tout compact de $M$ vers une fonction $f \in L_{1}^{2}(g)$ telle que $\gamma=\beta+\mathrm{d} f$ soit $g$-harmonique,

$$
\int_{M} f \mathrm{vol}^{g}=0 \quad \text { et } \quad\|f\|_{L_{1}^{2}} \leq c\|\beta\|_{L^{2}(g)} .
$$

Démonstration. - Le représentant $g_{j}$-harmonique de $b$ s'écrit sous la forme $\gamma_{j}=\beta+\mathrm{d} f_{j}$, où $f_{j}$ vérifie

$$
\int_{M} f_{j} \mathrm{vol}^{g_{j}}=0, \quad \Delta^{g_{j}} f_{j}=-\mathrm{d}^{* g_{j}} \beta
$$

d'après le lemme 7 ,

$$
c \int\left|f_{j}\right|^{2} \mathrm{vol}^{g_{j}} \leq \int\left|\mathrm{d} f_{j}\right|^{2} \mathrm{vol}^{g_{j}}=-\int f_{j} \mathrm{~d}^{*_{j}} \beta \mathrm{vol}^{g_{j}}=-\int\left\langle\mathrm{d} f_{j}, \beta\right\rangle \mathrm{vol}^{g_{j}},
$$

et d'après l'inégalité de Cauchy-Schwarz, on en déduit un contrôle

$$
\sqrt{c}\left\|f_{j}\right\|_{L^{2}\left(g_{j}\right)} \leq\left\|\mathrm{d} f_{j}\right\|_{L^{2}\left(g_{j}\right)} \leq\|\beta\|_{L^{2}\left(g_{j}\right)} .
$$

BULletin DE LA SOCiÉtÉ MATHÉMATiQUe DE FRANCE 
Comme $\beta$ est à support compact, il est clair que $\|\beta\|_{L^{2}\left(g_{j}\right)}=\|\beta\|_{L^{2}(g)}$ pour $j$ suffisamment grand. Sur un compact $K$ de $M$ la norme $L_{1}^{2}$ de $f_{j}$ est donc uniformément bornée. Quitte à extraire une sous suite, on peut supposer que $f_{j}$ converge sur tout compact de $M$ vers une limite faible $f \in L_{1}^{2}(g)$ qui vérifie

$$
\begin{gathered}
\sqrt{c}\|f\|_{L^{2}(g)} \leq\|\beta\|_{L^{2}(g)}, \quad\|\mathrm{d} f\|_{L^{2}(g)} \leq\|\beta\|_{L^{2}(g)}, \\
\Delta^{g} f=-\mathrm{d}^{* g} \beta, \quad \int_{M} f \mathrm{vol}^{g}=0 .
\end{gathered}
$$

Seule la dernière identité n'est pas évidente : on sait que $f \in L^{2}$ et que $\left\|f_{j}\right\|_{L^{2}\left(g_{j}\right)}$ uniformément bornée; d'après le lemme 3 , on peut donc choisir un compact $K$ de $M$ suffisamment grand, tel que

$$
\begin{gathered}
\int_{M \backslash K}|f| \mathrm{vol}^{g} \leq \operatorname{vol}^{g}(M \backslash K)^{\frac{1}{2}}\left(\int_{M \backslash K}|f|^{2} \mathrm{vol}^{g}\right)^{\frac{1}{2}} \leq \epsilon, \\
\left|\int_{M \backslash K} f_{j} \mathrm{vol}^{g_{j}}\right| \leq \operatorname{vol}^{g_{j}}(M \backslash K)^{\frac{1}{2}}\left\|f_{j}\right\|_{L^{2}\left(g_{j}\right)} \leq \epsilon \quad \text { pour tout } j .
\end{gathered}
$$

Par compacité de l'inclusion $L_{1}^{2} \hookrightarrow L^{2},\left(f_{j}\right)$ converge vers $f$ au sens $L^{2}$-fort sur $K$. Alors

$$
\int_{K} f \mathrm{vol}^{g}=\lim \int_{K} f_{j} \mathrm{vol}^{g_{j}}=-\lim \int_{M \backslash K} f_{j} \mathrm{vol}^{g_{j}},
$$

d'où $\mid \int_{K} f$ vol $^{g} \mid \leq \epsilon$. Maintenant,

$$
\left|\int_{M} f \mathrm{vol}^{g}\right| \leq\left|\int_{K} f \mathrm{vol}^{g}\right|+\int_{M \backslash K}|f| \mathrm{vol}^{g} \leq 2 \epsilon,
$$

d'où $\int_{M} f$ vol $^{g}=0$.

Par régularité elliptique la convergence est en réalité $C^{\infty}$ sur tout compact de $M$. Finalement $\gamma=\beta+\mathrm{d} f$ est une 1 -forme $g$-harmonique $L^{2}$ qui représente la classe de cohomologie définie par $b$ en cohomologie $L^{2}$ d'où le résultat.

Comportement à l'infini. — Nous avons également un contrôle près de l'infini sur les $\gamma_{j}$ de la proposition 3.2 à l'aide du lemme suivant :

LEMME 8. - En reprenant les notations de la proposition 3.2, pour tout $\epsilon>0$, il existe un $T$ suffisamment grand tel que

$$
\int_{t \geq T}|\gamma|^{2} \mathrm{vol}^{g} \leq \epsilon \quad \text { et } \quad \int_{t \geq T}\left|\gamma_{j}\right|^{2} \mathrm{vol}^{g_{j}} \leq \epsilon .
$$

Démonstration. - Reprenons la démonstration de la proposition 3.2 ainsi que ses notations. Par hypothèse, $\beta$ est à support compact, donc pour $T$ assez grand, on a $\gamma_{j}=\beta+\mathrm{d} f_{j}=\mathrm{d} f_{j}$ d'où

$$
\int_{t \geq T}\left|\gamma_{j}\right|^{2} \operatorname{vol}^{g_{j}}=\int_{t \geq T}\left|\mathrm{~d} f_{j}\right|^{2} \operatorname{vol}^{g_{j}}
$$

TOME $130-2002-\mathrm{N}^{\mathrm{O}} 3$ 
En intégrant par parties, il vient

$$
\begin{aligned}
\int_{t \geq T}\left|\mathrm{~d} f_{j}\right|^{2} \mathrm{vol}^{g_{j}} & =\int_{t \geq T} \mathrm{~d} f_{j} \wedge *_{j} \mathrm{~d} f_{j}=\int_{t \geq T} \mathrm{~d}\left(f_{j} *_{j} \mathrm{~d} f_{j}\right)-\int_{t \geq T} f_{j} \mathrm{~d} *_{j} \mathrm{~d} f_{j} \\
& =\int_{t=T} f_{j} *_{j} \mathrm{~d} f_{j}+\int_{t \geq T}\left\langle\Delta^{g_{j}} f_{j}, f_{j}\right\rangle \mathrm{vol}^{g_{j}} .
\end{aligned}
$$

Comme nous nous sommes placé hors du support de $\beta, \Delta^{g_{j}} f_{j}=\Delta^{g_{j}} f_{j}+\mathrm{d}^{*_{j}} \beta=$ $\Delta^{g_{j}} \gamma_{j}=0$, d'où

$$
\int_{t \geq T}\left|\mathrm{~d} f_{j}\right|^{2} \mathrm{vol}^{g_{j}}=\int_{t=T} f_{j} *_{j} \mathrm{~d} f_{j} .
$$

Le même calcul reste valable pour la limite $\gamma=\beta+\mathrm{d} f$ car $f \in L_{1}^{2}$. Choisissons un $T$ suffisamment grand, de sorte que $\int_{t \geq T}|\mathrm{~d} f|^{2} \mathrm{vol}^{g} \leq \frac{1}{2} \epsilon$. Alors comme $f_{j}$ tend vers $f$ au sens $C^{\infty}$ sur tout compact on a

$$
\int_{t=T} f_{j} *_{j} \mathrm{~d} f_{j} \longrightarrow \int_{t=T} f * \mathrm{~d} f \text { pour } j \rightarrow \infty
$$

soit

$$
\int_{t \geq T}\left|\mathrm{~d} f_{j}\right|^{2} \mathrm{vol}^{g_{j}} \longrightarrow \int_{t \geq T}|\mathrm{~d} f|^{2} \mathrm{vol}^{g} \text { pour } j \rightarrow \infty
$$

dont on déduit le lemme.

3.3.3. Inégalité de Poincaré pour les 1-formes. - Afin de démontrer un résultat de convergence des 2-formes harmoniques ( $c f$. prop. 3.4) analogue à celui des 1-formes, nous commençons par démontrer la proposition suivante :

Proposition 3.3. - Supposons que $M$ soit une variété avec des bouts paraboliques vérifiant la condition $\alpha \in \mathbb{Q}$. Soit $g$ une métrique asymptotique au modèle local et $g_{j}$ la suite d'approximations associée. Il existe une constante $c>0$ telle que pour toutes les métriques $g_{j}$, et toute 1 -forme orthogonale aux formes $g_{j}$ harmoniques, on ait :

$$
\int_{M}\left(|\mathrm{~d} \beta|^{2}+\left|\mathrm{d}^{*_{j}} \beta\right|^{2}\right) \mathrm{vol}^{g_{j}} \geq c \int_{M}|\beta|^{2} \mathrm{vol}^{g_{j}},
$$

les normes étant prises par rapport à la métrique $g_{j}$. L'inégalité est également vérifiée pour la métrique $g$ et toute 1 -forme $\beta \in L_{1}^{2}(g)$ orthogonale aux formes g-harmoniques $L^{2}$.

Remarque. - Cette inégalité permet de développer la théorie de Hodge $L^{2}$ pour la métrique $g$ dans le cas des 2-formes. Ainsi, toute classe de $\mathrm{H}_{L^{2}}^{2}(M)$ admet un unique représentant $g$-harmonique $L^{2}$.

Nous avons besoin d'un lemme préliminaire avant de commence la démonstration.

BULletin DE LA SOCiÉtÉ MATHÉmATiQUe DE FRANCE 
LEMME 9. - Soit $\hat{g}$ un modèle local de métrique dont les bouts vérifient la condition de rationalité. Il existe une constante $T$ suffisamment grande telle que pour toute métrique $g=\hat{g}$ ou $\hat{g}_{j}$, et toute 1 -forme $\beta$ sur $\left[t_{1}, t_{2}\right] \times N$ avec $t_{1}, t_{2} \geq T$, on ait :

$$
h_{0}^{2} \int_{\left[t_{1}, t_{2}\right] \times N}|\beta|^{2} \operatorname{vol}+h_{0} \int_{\partial\left[t_{1}, t_{2}\right] \times N}|\beta|^{2} \operatorname{vol}^{N} \leq \int_{\left[t_{1}, t_{2}\right] \times N}\left(|\nabla \beta|^{2}+\operatorname{Ric}(\beta, \beta)\right) \text { vol, }
$$

les normes étant prises par rapport à la métrique $g$.

Démonstration. - Soit $g$ une métrique de la forme $g=\mathrm{d} t^{2}+g_{t}, \operatorname{sur}\left[t_{1}, t_{2}\right] \times N$. On décompose les 1 -formes en $\beta=f \mathrm{~d} t+\mu$, avec $i_{\partial_{t}} \mu=0$. Alors la connexion de Levi-Civita se décompose en $\nabla \beta=\mathrm{d} t \otimes \nabla_{\partial_{t}} \beta+\nabla_{\mid N} \beta$, où $\nabla_{\mid N} \beta$ est la restriction de $\nabla \beta$ à une section de $T^{*} N \otimes \Omega^{1}\left(\left[t_{1}, t_{2}\right] \times N\right)$. Calculons ce terme plus précisément : notons $\nabla^{N}$ la connexion de Levi-Civita induite sur $N$ par $g$ et $\mathrm{d}^{N}$ la différentielle suivant la tranche, alors

$$
\nabla_{\mid N} \beta=\mathrm{d}^{N} f \otimes \mathrm{d} t+f \nabla_{\mid N} \mathrm{~d} t+\nabla^{N} \mu+\mathbb{I}(\mu) \otimes \mathrm{d} t .
$$

Or $\nabla_{\mid N} \mathrm{~d} t=\left\langle., \nabla_{\mid N} \partial_{t}\right\rangle=-\left\langle\nabla_{\mid N} ., \partial_{t}\right\rangle=-\mathbb{I}$, donc

$$
\nabla \beta=\mathrm{d} t \otimes \nabla_{\partial_{t}} \beta+\left(\mathrm{d}^{N} f+\mathbb{I}(\mu)\right) \otimes \mathrm{d} t-f \mathbb{I}+\nabla^{N} \mu .
$$

Appliquons ce calcul dans le cas où $g$ est égale au modèle local $\hat{g}$, ou à $\hat{g}_{j}$. La métrique $g_{t}$ sur la tranche $N \simeq S^{1} \times \mathbb{C P}^{1}$ s'exprime en prenant par exemple la coordonnée $u$ de projection stéréographique définie par $v=1$,

$$
g_{t}=\varphi^{2}(t) \mathrm{d} \theta^{2}+\frac{4 / c}{\left(1+|u|^{2}\right)^{2}}|\mathrm{~d} u-i \alpha u \mathrm{~d} \theta|^{2} ;
$$

la deuxième forme fondamentale est donnée par

$$
\mathbb{I}=-\frac{1}{2} g_{t}=-\frac{\partial_{t} \varphi}{\varphi}(\varphi \mathrm{d} \theta)^{2} \quad \text { et } \quad h=\frac{1}{2} \operatorname{tr}_{g} \mathbb{I}=-\frac{1}{2} \frac{\partial_{t} \mathrm{vol}^{g_{t}}}{\mathrm{vol}^{g_{t}}}=-\frac{1}{2} \frac{\partial_{t} \varphi}{\varphi} .
$$

En notant $\mu=f_{2} \varphi \mathrm{d} \theta+\eta$ avec $i_{\mathcal{X}_{\theta}} \eta=0$ et $\mathrm{d}^{\mathbb{C P}^{1}} f$ la partie de $\mathrm{d} f$ orthogonale à $\mathrm{d} \theta$ et $\mathrm{d} t$, la connexion de Levi-Civita sur la tranche est égale à

$\nabla^{N} \mu=\left(\varphi^{-1} \mathcal{X}_{\theta} \cdot f_{2}\right) \varphi \mathrm{d} \theta \otimes \varphi \mathrm{d} \theta+\left(\mathrm{d}^{\mathbb{C P}} f_{2}\right) \otimes \varphi \mathrm{d} \theta+\varphi \mathrm{d} \theta \otimes\left(\mathcal{L}_{\varphi^{-1}} \mathcal{X}_{\theta} \eta\right)+\nabla^{\mathbb{C P}} \eta$

Finalement on peut écrire

$$
\begin{array}{r}
\nabla \beta=\mathrm{d} t \otimes \nabla_{\partial_{t}} \beta+\left(\varphi^{-1} \mathcal{X}_{\theta} \cdot f+f_{2}\right) \varphi \mathrm{d} \theta \otimes \mathrm{d} t+\left(\varphi^{-1} \mathcal{X}_{\theta} \cdot f_{2}-f\right) \varphi \mathrm{d} \theta \otimes \varphi \mathrm{d} \theta \\
+\left(\mathrm{d}^{\mathbb{C P}} f\right) \otimes \mathrm{d} t+\left(\mathrm{d}^{\mathbb{C P}^{1}} f_{2}\right) \otimes \varphi \mathrm{d} \theta+\varphi \mathrm{d} \theta \otimes\left(\mathcal{L}_{\varphi^{-1}} \mathcal{X}_{\theta} \eta\right)+\nabla^{\mathbb{C P}} \eta
\end{array}
$$

or $\operatorname{Ric}^{g}(\beta, \beta)=\operatorname{Ric}^{\mathbb{C P}^{1}}(\eta, \eta)-\left(\partial_{t}^{2} \varphi / \varphi\right)\left(|f|^{2}+\left|f_{2}\right|^{2}\right)$, où $\operatorname{Ric}^{\mathbb{C P}^{1}}$ est la courbure provenant de la métrique de Fubini-Study; on en déduit l'inégalité suivante

TOME $130-2002-\mathrm{N}^{\mathrm{O}} 3$ 
en faisant une intégration par parties sur la tranche avec le terme en $\left|\nabla^{\mathbb{C P}} \eta\right|^{2}$ et en en utilisant le fait que par construction $-\varphi^{-1} \partial_{t}^{2} \varphi \geq-1$ :

$$
\begin{aligned}
\int_{N}|\nabla \beta|^{2} & +\operatorname{Ric}^{g}(\beta, \beta) \geq \int_{N}\left|\nabla_{\partial_{t}} \beta\right|^{2}+\left|\varphi^{-1} \mathcal{X}_{\theta} \cdot f\right|^{2}+\left|\varphi^{-1} \mathcal{X}_{\theta} \cdot f_{2}\right|^{2} \\
& +2\left\langle\varphi^{-1} \mathcal{X}_{\theta} \cdot f, f_{2}\right\rangle-2\left\langle f, \varphi^{-1} \mathcal{X}_{\theta} \cdot f_{2}\right\rangle+\left|\mathrm{d}^{\mathbb{C P}} f\right|^{2}+\left|\mathrm{d}^{\mathbb{C P}} f_{2}\right|^{2} .
\end{aligned}
$$

Nous allons maintenant montrer que les termes mixtes de (21) sont contrôlés. En intégrant par parties sur $N$ il vient

$$
\int_{N}\left\langle\varphi^{-1} \mathcal{X}_{\theta} \cdot f, f_{2}\right\rangle \operatorname{vol}^{g}=-\int_{N}\left\langle f, \varphi^{-1} \mathcal{X}_{\theta} \cdot f_{2}\right\rangle \operatorname{vol}^{g}
$$

dès que $f$ ou $f_{2}$ est constante suivant $\mathcal{X}_{\theta}$, ces intégrales sont nulles et on conclut à l'aide du lemme 4. On supposera à partir de maintenant que $f$ et $f_{2}$ sont orthogonales aux fonctions constantes suivant $\mathcal{X}_{\theta}$. Dans le cas d'un bout parabolique dont les poids vérifient la condition de rationnalité, le champ de vecteurs $\mathcal{X}_{\theta}$ est périodique et donne une structure de $S^{1}$-fibré. Dans le revêtement à $q$ feuillets $p^{q}: \Delta_{a / q}^{*} \times \mathbb{C P}^{1} \rightarrow \mathcal{B}$, les orbites de $\mathcal{X}_{\theta}$ correspondent aux cercles centrés en 0 de $\Delta_{a / q}^{*}$.

En utilisant la décomposition en série de Fourier sur ce revêtement local, $f=\sum_{k} c_{k} \mathrm{e}^{k i \theta / q}$, on voit que la condition d'être orthogonale aux constantes se traduit par $c_{0}=0$. Alors

$$
\int_{N}\left|\mathcal{X}_{\theta} \cdot f\right|^{2}=\int_{N} \sum_{k}\left|\frac{k}{q} c_{k}\right|^{2} \geq \frac{1}{q^{2}} \int_{N}|f|^{2},
$$

d'où

$$
\int_{N}\left|\varphi^{-1} \mathcal{X}_{\theta} \cdot f\right|^{2} \geq \frac{1}{(q \varphi)^{2}} \int_{N}|f|^{2}, \quad \int_{N}\left|\varphi^{-1} \mathcal{X}_{\theta} \cdot f_{2}\right|^{2} \geq \frac{1}{(q \varphi)^{2}} \int_{N}\left|f_{2}\right|^{2} .
$$

Puisque $\varphi(t) \leq \mathrm{e}^{-t} \rightarrow 0$, on en déduit que pour $t$ suffisamment grand, les termes mixtes de $(21)$ sont très bien contrôlés par les termes en $\left|\varphi^{-1} \mathcal{X}_{\theta} \cdot f\right|^{2}$ et $\left|\varphi^{-1} \mathcal{X}_{\theta} \cdot f_{2}\right|^{2}$, d'où

$$
\int_{N}|\nabla \beta|^{2}+\operatorname{Ric}^{g}(\beta, \beta) \geq \int_{N}\left|\nabla_{\partial_{t}} \beta\right|^{2}
$$

et le lemme 4 nous permet de conclure.

REMARQUE. - Les hypothèses que nous devons faire dans ce lemme sur la métrique sont assez faibles ; par exemple, la courbure positive du facteur $\mathbb{C P}^{1}$ n'intervient pas. On peut ainsi aisément corriger au passage la démonstration de l'inégalité de Poincaré sur les 1-formes de [3], où une erreur s'est glissée dans les termes mixtes de la formule de Bochner du lemme 4.5. 
Ce lemme reste vrai (en modifiant légèrement les constantes) si l'on remplace $\hat{g}$ et $\hat{g}_{j}$ par une métrique asymptotique au modèle local $g$ et ses approximations $g_{j}$.

Corollaire 4. - Soit $g$ une métrique asymptotique au modèle local $\hat{g}$. Il existe des constantes $T, c>0$ telles que pour toute métrique $g$ ou $g_{j}$, et toute 1 -forme $\beta$ sur $\left[t_{1}, t_{2}\right] \times N$ avec $t_{1}, t_{2} \geq T$, on ait :

$h_{0}^{2} \int_{\left[t_{1}, t_{2}\right] \times N}|\beta|^{2} \mathrm{vol}+h_{0} \int_{\partial\left[t_{1}, t_{2}\right] \times N}|\beta|^{2} \operatorname{vol}^{N} \leq c \int_{\left[t_{1}, t_{2}\right] \times N}\left(|\nabla \beta|^{2}+\operatorname{Ric}(\beta, \beta)\right) \mathrm{vol}$.

Démonstration. - Comme les métriques $\hat{g}_{j}$ et $g_{j}$ sont égales pour $t \geq j+\frac{1}{2}$, il nous suffit de vérifier le corollaire pour la métrique $g$. Il faut voir que l'inégalité est perturbée lorsque on passe de $\hat{g}$ à $g$ par des termes en $\epsilon(T) \cdot O\left(\|\beta\|_{L_{1}^{2}}^{2}\right)$, avec $\epsilon(T) \rightarrow 0$ quand $T \rightarrow \infty$. Ce n'est pas complètement évident pour les termes de bord; pour la métrique $\hat{g}$

$$
\int_{t^{\prime}}|\beta|^{2} \operatorname{vol}^{\hat{g}_{t}}=\int_{t \leq t^{\prime}}\left\langle\beta, \nabla_{\partial_{t}} \beta\right\rangle-2 h|\beta|^{2} \operatorname{vol}^{\hat{g}}
$$

et on en déduit le corollaire.

Nous avons maintenant fait un premier pas dans la démonstration de la proposition 3.3 grâce au corollaire suivant :

Corollaire 5. - Il existe une constante $c>0$ et $T$ suffisamment grand, tels que pour toute 1 -forme lisse $\beta$ sur le bout parabolique compactifié $\overline{\mathcal{B}}$ nulle en $t=T$ on ait

$$
\int_{t \geq T}\left(|\mathrm{~d} \beta|^{2}+\left|\mathrm{d}^{*_{j}} \beta\right|^{2}\right) \operatorname{vol}^{g_{j}} \geq c \int_{t \geq T}|\beta|^{2} \operatorname{vol}^{g_{j}}
$$

les normes étant prises par rapport à la métrique $g_{j}$. Cette inégalité est également valable pour la métrique $g$, et pour une 1 -forme $\beta \in L_{1}^{2}(g)$ définie sur $\mathcal{B}$ et nulle en $t=T$.

Démonstration. - On applique le corollaire $4 \operatorname{sur}\left[T, T_{j}\right] \times N$ pour la métrique $g_{j}$. Le terme de bord est nul par hypothèse pour $t=T$. Il est nul également pour $t=T_{j}$ car le volume de cette tranche est égal à 0 . Dans le cas de la métrique $g$ on fait tendre $T_{j}$ vers l'infini et le terme de bord correspondant tend vers 0 car $\beta \in L_{1}^{2}$. On en déduit le corollaire par la formule de Bochner $\Delta=\nabla^{*} \nabla+$ Ric en intégrant par parties.

Preuve de la proposition 3.3. — Supposons la proposition fausse; on en déduit une suite de 1 -formes $\beta_{j}$ est orthogonales aux formes $g_{j}$-harmoniques telles que $\left\|\beta_{j}\right\|_{L^{2}\left(g_{j}\right)}=1,\left\|\mathrm{~d} \beta_{j}\right\|_{L^{2}\left(g_{j}\right)} \rightarrow 0$ et $\left\|\mathrm{d}^{*_{j}} \beta_{j}\right\|_{L^{2}\left(g_{j}\right)} \rightarrow 0$. Puisque $\left\|\beta_{j}\right\|_{L^{2}\left(g_{j}\right)}$ est bornée on peut en extraire une limite faible $\beta \in L_{1}^{2}(g)$ sur tout compact de $M$. La limite vérifie nécessairement $\left\|\mathrm{d} \beta_{j}\right\|_{L^{2}(g)}=\left\|\mathrm{d}^{*_{g}} \beta\right\|_{L^{2}(g)}=0$ et $\|\beta\|_{L^{2}(g)}=1$; $\beta$ est donc $g$-harmonique.

TOME $130-2002-\mathrm{N}^{\mathrm{O}} 3$ 
Montrons maintenant que $\beta$ est orthogonale aux formes $g$-harmoniques $L^{2}$. Soit $\gamma$ une 1 -forme $g$-harmonique $L^{2}$ et $\gamma_{j}$ les représentant $g_{j}$ harmoniques de $[\gamma]$. On décompose l'intégrale

$$
0=\int_{M}\left\langle\beta_{j}, \gamma_{j}\right\rangle \mathrm{vol}^{g_{j}}=\int_{M \backslash\{t \geq T\}}\left\langle\beta_{j}, \gamma_{j}\right\rangle \mathrm{vol}^{g_{j}}+\int_{t \geq T}\left\langle\beta_{j}, \gamma_{j}\right\rangle \mathrm{vol}^{g_{j}}
$$

et on majore

$$
\left|\int_{t \geq T}\left\langle\beta_{j}, \gamma_{j}\right\rangle \mathrm{vol}^{g_{j}}\right|^{2} \leq\left\|\beta_{j}\right\|_{L^{2}\left(g_{j}\right)}^{2} \int_{t \geq T}\left|\gamma_{j}\right|^{2} \mathrm{vol}^{g_{j}}=\int_{t \geq T}\left|\gamma_{j}\right|^{2} \mathrm{vol}^{g_{j}} .
$$

De même,

$$
\left|\int_{t \geq T}\langle\beta, \gamma\rangle \operatorname{vol}^{g}\right|^{2} \leq\|\beta\|_{L^{2}(g)}^{2} \int_{t \geq T}|\gamma|^{2} \operatorname{vol}^{g}=\int_{t \geq T}|\gamma|^{2} \operatorname{vol}^{g} .
$$

D'après le lemme 8 choisir un $T$ suffisamment grand tel que

$$
\left|\int_{t \geq T}\left\langle\beta_{j}, \gamma_{j}\right\rangle \mathrm{vol}^{g_{j}}\right| \leq \epsilon, \quad\left|\int_{t \geq T}\langle\beta, \gamma\rangle \mathrm{vol}^{g}\right| \leq \epsilon .
$$

D'après (23), on a $\left|\int_{M \backslash\{t \geq T\}}\left\langle\beta_{j}, \gamma_{j}\right\rangle \mathrm{vol}^{g_{j}}\right| \leq \epsilon$. Sur un compact $\gamma_{j} \rightarrow \gamma$ et $\beta_{j} \rightarrow \beta$ au sens $L^{2}$-fort par compacité de l'inclusion $L_{1}^{2} \hookrightarrow L_{2}$; en faisant tendre $j$ vers l'infini

$$
\int_{M \backslash\{t \geq T\}}\left\langle\beta_{j}, \gamma_{j}\right\rangle \operatorname{vol}^{g_{j}} \longrightarrow \int_{M \backslash\{t \geq T\}}\langle\beta, \mu\rangle \operatorname{vol}^{g}
$$

et il est facile d'en déduire que $\beta$ est orthogonale à $\gamma$. Finalement $\beta$ est à la fois harmonique et orthogonale aux formes harmoniques donc $\beta=0$.

Nous allons voir maintenant que nos hypothèses impliquent une contradiction. Choisissons un $T>0$ suffisamment grand afin d'être dans le cadre du corollaire 5. Soit $\chi(t)$ une fonction cut-off valant 1 sur le compact $M \backslash\{t \geq T\}$ et 0 hors d'un compact. Alors on découpe les $\beta_{j}$ en

$$
\left\|\beta_{j}\right\|_{L^{2}\left(g_{j}\right)}^{2}=1 \leq 2\left\|\chi \beta_{j}\right\|_{L^{2}\left(g_{j}\right)}^{2}+2\left\|(1-\chi) \beta_{j}\right\|_{L^{2}\left(g_{j}\right)}^{2},
$$

afin d'appliquer 5 à $(1-\chi) \beta_{j}$ :

$$
\begin{aligned}
c\left\|(1-\chi) \beta_{j}\right\|_{L^{2}\left(g_{j}\right)}^{2} & \leq\left\|\mathrm{d}(1-\chi) \beta_{j}\right\|_{L^{2}\left(g_{j}\right)}^{2}+\left\|\mathrm{d}^{*_{j}}(1-\chi) \beta_{j}\right\|_{L^{2}\left(g_{j}\right)}^{2} \\
& \leq\left\|\mathrm{d} \beta_{j}\right\|_{L^{2}\left(g_{j}\right)}^{2}+\left\|\mathrm{d}^{*_{j}} \beta_{j}\right\|_{L^{2}\left(g_{j}\right)}^{2}+2\left\|\left(\partial_{t} \chi\right) \beta_{j}\right\|_{L^{2}\left(g_{j}\right)}^{2} .
\end{aligned}
$$

Puisque, $\beta_{j}$ converge vers $\beta=0$ au sens $L^{2}$-fort sur tout compact de $M$ et que par hypothèse $\left\|\mathrm{d} \beta_{j}\right\|_{L^{2}\left(g_{j}\right)}$ et $\left\|\mathrm{d}^{*_{j}} \beta_{j}\right\|_{L^{2}\left(g_{j}\right)}$ tendent vers 0 , il en résulte que $\left\|\beta_{j}\right\|_{L^{2}\left(g_{j}\right)}$ tend vers 0 , ce qui contredit l'hypothèse $\left\|\beta_{j}\right\|_{L^{2}\left(g_{j}\right)}=1$.

BULLETIN DE LA SOCIÉtÉ MATHÉMATIQUE DE FRANCE 
3.3.4. Convergence des 2-formes harmoniques. - Comme dans le cas des 1formes, on va utiliser l'inégalité de Poincaré du lemme 9 afin d'étudier la convergence des représentants $g_{j}$-harmoniques d'une classe de cohomologie fixée.

Proposition 3.4. - Soit une classe de cohomologie $b \in \mathrm{H}_{\mathrm{DR}}^{2}(\bar{M})$. La suite $\gamma_{j}$ de ses représentants $g_{j}$-harmoniques sur $\bar{M}$ converge sur tout compact de $M$ vers un représentant $g$-harmonique $L^{2}$ de $b$.

Plus précisément : soit un représentant $w$ de b égal à $\lambda \mathrm{vol}^{\mathbb{C P}^{1}}$ près de l'infini sur le bout parabolique de $M$ (avec $\lambda \in \mathbb{R})$. On écrit alors $\gamma_{j}=w+\mathrm{d} \beta_{j}$, où $\beta_{j}$ est une 1-forme lisse sur $\bar{M}$ orthogonale aux formes $g_{j}$-harmoniques et telle que $\mathrm{d}^{*_{j}} \beta_{j}=0$. Alors $\beta_{j}$ converge sur tout compact de $M$ vers une 1 -forme $\beta$ dans $L_{1}^{2}(g)$ orthogonale aux formes g-harmoniques $L^{2}$, telle que $\gamma=w+\mathrm{d} \beta$ soit g-harmonique, $\mathrm{d}^{* g} \beta=0$ et $\|\beta\|_{L_{1}^{2}} \leq c\|w\|_{L^{2}(g)}$, où $c>0$ est un constante fixée.

Démonstration. - L'écriture de $\gamma_{j}$ sous la forme $\omega+\mathrm{d} \beta_{j}$ relève de la théorie de Hodge élémentaire sur $\bar{M}$ et du lemme de Poincaré 5 . On remarque dans un premier temps que la forme vol $\mathbb{C P}^{1}$ est harmonique relativement aux métriques $\hat{g}$ et $\hat{g}_{j}$. On en déduit le lemme suivant pour les métriques $g$ et $g_{j}$ :

LEMme 10. - Quel que soit $\epsilon>0$, il existe $T$ suffisamment grand tel que

$$
\int_{t \geq T}\left|\mathrm{~d}^{*_{g}} \mathrm{vol}^{\mathbb{C P}^{1}}\right|^{2} \mathrm{vol}^{g} \leq \epsilon \quad \text { et } \quad \int_{t \geq T}\left|\mathrm{~d}^{*_{j}} \mathrm{vol}^{\mathbb{C P}}\right|^{2} \mathrm{vol}^{g_{j}} \leq \epsilon .
$$

Par hypothèse, $\gamma_{j}=w+\mathrm{d} \beta_{j}$ est $g_{j}$-harmonique, donc $\mathrm{d}^{*_{j}} d \beta_{j}=-\mathrm{d}^{*_{j}} w$. En intégrant contre $\beta_{j}$, puis par inégalité de Cauchy-Schwarz, il vient

$$
\left\|\mathrm{d} \beta_{j}\right\|_{L^{2}\left(g_{j}\right)}^{2}=-\int\left\langle\beta_{j}, \mathrm{~d}^{*_{j}} \gamma\right\rangle \operatorname{vol}^{g_{j}} \leq\left\|\beta_{j}\right\|_{L^{2}\left(g_{j}\right)} \cdot\left\|\mathrm{d}^{*_{j}} w\right\|_{L^{2}\left(g_{j}\right)} ;
$$

par l'inégalité de Poincaré de la proposition 3.3, on en déduit que

$$
\left\|\beta_{j}\right\|_{L^{2}\left(g_{j}\right)}^{2} \leq c\left\|\mathrm{~d} \beta_{j}\right\|_{L^{2}\left(g_{j}\right)}^{2} \leq c\left\|\beta_{j}\right\|_{L^{2}\left(g_{j}\right)} \cdot\left\|\mathrm{d}^{*_{j}} w\right\|_{L^{2}\left(g_{j}\right)} .
$$

D'après le lemme $10,\left\|\mathrm{~d}^{*_{j}} w\right\|_{L^{2}\left(g_{j}\right)}$ est uniformément bornée, ce qui entraîne que $\left\|\beta_{j}\right\|_{L^{2}\left(g_{j}\right)}$ puis que $\left\|\mathrm{d} \beta_{j}\right\|_{L^{2}\left(g_{j}\right)}$ sont uniformément bornées. Comme de plus $\mathrm{d}^{*_{j}} \beta_{j}=0$, on en déduit que $\left\|\beta_{j}\right\|_{L_{1}^{2}\left(g_{j}\right)}$ est uniformément bornée. À partir de là, on peut extraire une limite faible $\beta \in L_{1}^{2}$ de $\beta_{j}$ sur tout compact de $M$ et terminer la démonstration comme dans le lemme 3.2.

Seul le fait que la limite $\beta$ soit orthogonale aux formes $g$-harmoniques $L^{2}$ n'est pas complètement évident. Fixons une classe de cohomologie $b \in \mathrm{H}_{L^{2}}(M)$. Soit $\omega_{j}$ la suite de ses représentants $g_{j}$-harmoniques et $\omega$ le représentant $g$ harmonique $L^{2}$. Nous allons voir que

$$
\int\left\langle\omega_{j}, \gamma_{j}\right\rangle \operatorname{vol}^{g_{j}} \longrightarrow \int\langle\omega, \gamma\rangle \operatorname{vol}^{g}
$$

TOME $130-2002-\mathrm{N}^{\mathrm{O}} 3$ 
puisque la suite de gauche est identiquement nulle par hypothèse, on en déduira que $\gamma$ est orthogonale à toute forme $g$-harmonique $L^{2}$. À ce point, nous avons besoin d'un nouveau lemme technique :

Lemme 11. - Pour tout $\epsilon>0$, et toute classe de cohomologie $b \in \mathrm{H}_{L^{2}}^{2}(M)$, il existe un $T$ suffisamment grand tel que avec les notations de 3.4 , on ait

$$
\int_{t \geq T}|\gamma|^{2} \mathrm{vol}^{g} \leq \epsilon \quad \text { et } \quad \int_{t \geq T}\left|\gamma_{j}\right|^{2} \mathrm{vol}^{g_{j}} \leq \epsilon
$$

Démonstration du lemme. - En utilisant le lemme 10, on en déduit pour tout $\epsilon>0$, que pour $T$ suffisamment grand, on a

$$
\left\|\beta_{j}\right\|_{L^{2}\left(g_{j}\right)}\left(\int_{t \geq T}\left|\mathrm{~d}^{* j} w\right|^{2} \mathrm{vol}^{g_{j}}\right)^{1 / 2} \leq \frac{\epsilon}{2} \quad \text { pour tout } j .
$$

En intégrant par parties, il vient :

$$
\int_{t \geq T}\left|\mathrm{~d} \beta_{j}\right|^{2} \mathrm{vol}^{g_{j}}=-\int_{t \geq T}\left\langle\mathrm{~d}^{*_{j}} w, \beta_{j}\right\rangle \mathrm{vol}^{g_{j}}+\int_{t=T} \beta_{j} \wedge *_{j} \mathrm{~d} \beta_{j},
$$

d'où la majoration

$$
\begin{aligned}
\int_{t \geq T}\left|\mathrm{~d} \beta_{j}\right|^{2} \mathrm{vol}^{g_{j}} & \leq\left|\int_{t \geq T}\left\langle\mathrm{~d}^{*_{j}} \mathrm{~d} \beta_{j}, \beta_{j}\right\rangle \mathrm{vol}^{g_{j}}\right|+\left|\int_{t=T} \beta_{j} \wedge *_{j} \mathrm{~d} \beta_{j}\right| \\
& \leq\left\|\beta_{j}\right\|_{L^{2}\left(g_{j}\right)}\left(\int_{t \geq T}\left|\mathrm{~d}^{*_{j}} w\right|^{2} \mathrm{vol}^{g_{j}}\right)^{1 / 2}+\left|\int_{t=T} \beta_{j} \wedge *_{j} \mathrm{~d} \beta_{j}\right| \\
& \leq \frac{\epsilon}{2}+\left|\int_{t=T} \beta_{j} \wedge *_{j} \mathrm{~d} \beta_{j}\right| .
\end{aligned}
$$

Puisque $\beta_{j}$ converge vers $\beta$ au sens $C^{\infty}$ sur tout compact de $M$, le terme $\int_{t=T} \beta_{j} \wedge *_{j} \mathrm{~d} \beta_{j}$ converge vers $\int_{t=T} \beta \wedge * \mathrm{~d} \beta$ lorsque $j$ tend vers l'infini; comme $\beta \in L_{1}^{2}(g)$, en utilisant la formule (25) pour la métrique $g$ et le lemme 10 , on peut choisir une tranche $t=T$ près de l'infini telle que $\left|\int_{t=T} \beta \wedge * \mathrm{~d} \beta\right| \leq \frac{1}{4} \epsilon$. Donc pour $j$ suffisamment grand $\left|\int_{t \geq T} \beta_{j} \wedge * \mathrm{~d} \beta_{j}\right| \leq \frac{1}{2} \epsilon$, d'où le lemme.

Fin de la démonstration de la proposition 3.4. - Comme $\left\|\beta_{j}\right\|_{L^{2}\left(g_{j}\right)}$ est uniformément bornée, on peut choisir d'après le lemme 11 un compact $K$ tel que

$$
\left|\int_{M \backslash K}\left\langle\gamma_{j}, \beta_{j}\right\rangle \mathrm{vol}^{g_{j}}\right| \leq\left.\left.\left\|\beta_{j}\right\|_{L^{2}\left(g_{j}\right)}\left|\int_{M \backslash K}\right| \gamma_{j}\right|^{2} \mathrm{vol}^{g_{j}}\right|^{1 / 2} \leq \frac{\epsilon}{2}
$$

avec de plus $\mid \int_{M \backslash K}\left\langle\gamma_{j}, \beta_{j}\right\rangle$ vol $^{g_{j}} \mid \leq \frac{1}{2} \epsilon$. On découpe les intégrales de (24) en deux morceaux suivant $M \backslash K$ où on a le contrôle ci-dessus et suivant $K$ où la convergence de $\omega_{j}$ et de $\gamma_{j}$ est $C^{\infty}$; on en déduit facilement (24). 
Convergence à l'infini. - Les représentants $g_{j}$-harmoniques $\gamma_{j}$ et $\omega_{j}$ de deux classes cohomologie fixées de $\mathrm{H}_{L^{2}}^{2}(\bar{M})$ convergent sur tout compact de $M$ vers les représentants $g$-harmoniques $L^{2}, \gamma$ et $\omega$. Une autre conséquence intéressante du lemme 11 est la suivante :

$$
\int_{M}\left\langle\gamma_{j}, \omega_{j}\right\rangle \operatorname{vol}^{g_{j}} \longrightarrow \int_{M}\langle\gamma, \omega\rangle \operatorname{vol}^{g} \quad \text { lorsque } j \rightarrow \infty \text {. }
$$

L'étoile de Hodge associée à $g$ induit un décomposition des 2-formes $g$-harmoniques $L^{2}$ en leur partie autoduale et anti-autoduale. On en déduit la décomposition correspondante de $\mathrm{H}_{L^{2}}^{2}(M)$ en

$$
\mathrm{H}_{L^{2}}^{2}(M)=H^{+_{g}} \oplus H^{-g} .
$$

Par ailleurs nous avons les décompositions usuelles pour les métriques $g_{j}$ sur la variété compactifiée $\bar{M}$

$$
\mathrm{H}_{\mathrm{DR}}^{2}(\bar{M})=H^{+_{j}} \oplus H^{-{ }_{j}} .
$$

De (26), on déduit alors le corollaire suivant :

Corollaire 6. - Soit une classe de cohomologie $b \in \mathrm{H}_{L^{2}}^{2}(M)$, alors

$$
b^{+j} \longrightarrow b^{+} \text {et } b^{-j} \longrightarrow b^{-} \text {. }
$$

Démonstration. - Soit $\left(\gamma_{j}\right)$ les représentants $g_{j}$-harmoniques de $b$ et $\gamma$ le représentant $g$-harmonique $L^{2}$. Soit $\left(\gamma^{+}\right)_{j}$ les représentants $g_{j}$-harmoniques de $b^{+}$. Par la proposition 3.4, la suite $\left(\gamma^{+}\right)_{j}$ converge sur tout compact vers son représentant $g$-harmonique qui est par hypothèse $\gamma^{+}$. Parce que les métriques $g_{j}$ convergent vers $g$ sur tout compact on en déduit que $\gamma_{j}^{+{ }_{j}} \rightarrow \gamma^{+}$sur tout compact. Alors pour toute classe de cohomologie $a \in \mathrm{H}_{L^{2}}^{2}(M)$, en notant $\omega_{j}$ la suite de ses représentants $g_{j}$-harmoniques, on a

$$
\left(b^{+}-b^{+_{j}}\right) \cdot a=\left[\left(\gamma^{+}\right)_{j}-\gamma_{j}^{+j}\right] \cdot\left[\omega_{j}\right] \leq\left\|\left(\gamma^{+}\right)_{j}-\gamma_{j}^{+{ }_{j}}\right\|_{L^{2}\left(g_{j}\right)} \cdot\left\|\omega_{j}\right\|_{L^{2}\left(g_{j}\right)} \cdot
$$

D'après la proposition 3.4, $\left\|\omega_{j}\right\|_{L^{2}\left(g_{j}\right)}$ est borné; on en déduit que $\left(b^{+}-b^{+}\right) \cdot a$ tend vers zéro d'après (26). Puisque le cup produit est une forme quadratique non dégénérée, ceci implique que $b^{+}-b^{+}{ }_{j}$ tend vers 0 dans $\mathrm{H}_{L^{2}}^{2}(M)$, d'où le corollaire.

\section{4. Équations de Seiberg-Witten}

Soit $M$ une surface complexe à bouts paraboliques munie d'une métrique $g$ asymptotique au modèle local $\hat{g}$. Nous commençons par expliquer comment généraliser la démonstration de Le Brun [4] à ce cadre de volume fini au $§ 4.1$, puis comment obtenir une solution des équations de Seiberg-Witten pour $g$ par un procédé de convergence au $\S 4.3$ dans le cas où les poids des bouts paraboliques vérifient la condition de rationalité.

TOME $130-2002-\mathrm{N}^{\mathrm{O}} 3$ 
4.1. Équations de Seiberg-Witten et bouts paraboliques. - La surface complexe $M$ est munie d'une structure $\operatorname{spin}^{c}$ canonique de fibré déterminant $L=K_{M}^{-1}$ avec les fibrés de spineurs

$$
W^{+}=\Lambda^{0,0} M \oplus \Lambda^{0,2} M \quad \text { et } \quad W^{-}=\Lambda^{0,1} M
$$

munis d'une action de Clifford de TM.

Soit $g$ une métrique asymptotique au modèle local sur $M$ et $A$ une connexion unitaire sur $M$. On en déduit un opérateur de Dirac $\mathrm{D}_{A}$ et les équations de Seiberg-Witten s'écrivent :

$$
\mathrm{D}_{A} \psi=0, \quad F_{A}^{+g}=q(\psi),
$$

où $\psi \in \Gamma\left(W^{+}\right)$et $q(\psi)$ est la partie dans trace de l'endomorphisme $\psi \otimes \psi^{*}$. La courbure $F_{A}^{+}$est une forme imaginaire autoduale et agit par multiplication de Clifford comme un endomorphisme hermitien sans trace, ce qui donne un sens à la deuxième équation.

4.1.1. Connexions $L_{1}^{2}$. - Sur les bouts paraboliques de $M$, le modèle local de métrique kählérienne $\hat{g}$ induit une connexion $\hat{A}$ sur le fibré anti-canonique $K_{M}^{-1}$ que l'on étend par partition de l'unité au fibré tout entier. On définit alors la classe de Chern $L^{2}$ du fibré anti-canonique de $M$ comme la classe de cohomologie de $\mathrm{H}_{L^{2}}^{2}(M)$

$$
\mathrm{L}^{2} c_{1}=\frac{i}{2 \pi}\left[F_{\hat{A}}\right] .
$$

Si $A$ est une connexion sur $K_{M}^{-1}$ telle que $A=\hat{A}+a$ avec $a$ et d $a \in L^{2}$, alors $\mathrm{L}^{2} c_{1}=\frac{i}{2 \pi}\left[F_{A}\right]$. En particulier si $g$ est une métrique de Kähler asymptotique au modèle local sur les bouts paraboliques de $M$, elle induit alors une connexion $A^{g}$ sur $K_{M}^{-1}$ telle que $A^{g}-\hat{A} \in L_{1}^{2}$ d'où $\mathrm{L}^{2} c_{1}=\frac{i}{2 \pi}\left[F_{A^{g}}\right]$.

4.1.2. Un lemme clef. - Le lemme utilisé par Le Brun dans [4] pour démontrer le théorème $2.1 \mathrm{a}$ un analogue dans notre contexte de volume fini à condition de faire des hypothèses de régularité à l'infini sur la solution des équations de Seiberg-Witten (28).

Lemme 12. - Supposons que les équations de Seiberg-Witten non perturbées (28) sur $M$, associées à une métrique asymptotique au modèle local $g$, admettent une solution $(A, \psi)$ telle que $\psi \not \equiv 0, A=\hat{A}+a$, avec $a \in L_{1}^{2}$, et $\psi \in L_{1}^{2}$. Alors

$$
\frac{1}{32 \pi^{2}} \int_{M} s_{g}^{2} \mathrm{vol}^{g} \geq\left(\mathrm{L}^{2} c_{1}^{+g}\right)^{2}
$$

En outre, le cas d'égalité est réalisé si et seulement si $\nabla_{A} \varphi=0$ et $s=\mathrm{C}^{\mathrm{te}}<0$. Dans ce cas, $g$ est une métrique de Kähler relativement à une structure complexe $J$ induite par la métrique $g$ et la forme de Kähler $F_{A}^{+} / \sqrt{2}\left|F_{A}^{+}\right|$.

BULletin DE LA SOCiÉtÉ MATHÉMATIQUE DE FRANCE 
Démonstration. - Identique à celle à celle des surfaces compactes, basée sur la formule de Lichnerowicz ( $c f$. [4]), car les hypothèses de régularité sur $(A, \psi)$ nous permettent de faire les intégrations par parties.

4.2. Démonstration du théorème 2.1. - Dans tout le $\S 4.2, M=\mathbb{P}(\mathcal{E})_{\Sigma}$ est une surface réglée obtenue à partir d'un fibré parabolique $\mathcal{E}$. Pour simplifier, on supposera que $M$ possède au moins un bout parabolique et on se réfèrera à [6] et [4] pour les démonstrations dans le cas compact.

4.2.1. Une antipodie sur $M$. - Soit $h$ la métrique hermitienne sur $\mathcal{E}$ adaptée à la structure parabolique qui nous a permis de construire le modèle local $\hat{g}$. On en déduit une métrique de Fubini-Study sur chaque fibre de $M$ et une antipodie $\xi: M \rightarrow \Sigma$, qui à chaque point d'une fibre de $M$ associe le point diamétralement opposé. L'antipodie $\xi$ est clairement une involution du fibré $M \rightarrow \Sigma$ renversant l'orientation et une isométrie pour le modèle local de métrique $\hat{g}$; on déduit de cette dernière propriété que si $g$ est asymptotique au modèle local, alors $\xi^{*} g$ est également asymptotique au modèle local et que $\xi$ agit donc sur la cohomologie $\mathrm{H}_{L^{2}}^{*}(M)$.

LEMME 13. - L'antipodie $\xi$ agit sur $\mathrm{H}_{L^{2}}^{2}(M)$ en échangeant les facteurs de la décomposition

$$
\mathrm{H}_{L^{2}}^{2}(M)=\mathrm{H}_{L^{2}}^{+}(M) \oplus \mathrm{H}_{L^{2}}^{-}(M)
$$

Démonstration. — Comme $\xi$ est un difféomorphisme de $M$ qui renverse l'orientation, on en déduit que pour des 2-formes $L^{2}, \gamma_{1}$ et $\gamma_{2}$,

$$
\int_{M} \xi^{*} \gamma_{1} \wedge \xi^{*} \gamma_{2}=-\int_{M} \gamma_{1} \wedge \gamma_{2}
$$

Donc pour $a, b \in \mathrm{H}_{L^{2}}^{2}(M)$, on a $\xi^{*} a \cdot \xi^{*} b=-a \cdot b$; autrement dit $\xi$ ne fait que changer le signe de la forme d'intersection. En utilisant le fait que $\xi$ est une involution, on en déduit que $a \cdot \xi^{*} a=0$. Puisque nous sommes dans la situation $b_{2}^{+}=b_{2}^{-}=1$, cette dernière identité implique le lemme.

Corollaire 7. - Les métrique $g$ et $\xi^{*} g$ définissent la même décomposition

$$
\mathrm{H}_{L^{2}}^{2}(M)=\mathrm{H}_{L^{2}}^{+}(M) \oplus \mathrm{H}_{L^{2}}^{-}(M)
$$

Démonstration. - Soit une classe de cohomologie $a \in \mathrm{H}_{L^{2}}^{+g}(M)$. D'après le lemme $13, \xi^{*} a \in \mathrm{H}_{L^{2}}^{-g}(M)$; soit $\gamma$ le représentant $L^{2}, g$-harmonique antiautodual de $\xi^{*} a$. Alors $\xi^{*} \gamma$ représente $a$ et c'est une forme $L^{2}, \xi^{*} g$-harmonique autoduale, d'où $a \in \mathrm{H}_{L^{2}}^{+\xi^{*} g}(M)$. Les autres inclusions se démontrent de la même façon.

TOME $130-2002-\mathrm{N}^{\mathrm{O}} 3$ 
4.2.2. Fin de la preuve. - Voici maintenant une proposition essentielle dont on déduit automatiquement que $\left(M, g^{\mathrm{K}}\right) \simeq \Sigma \times_{\rho} \mathbb{C P}^{1}$ (cf. [4]). Par le théorème de Mehta-Seshadri, il en résulte que $\mathcal{E}$ est polystable ce qui démontre le théorème 2.1 .

Proposition 4.1. - Sous les hypothèses du théorème 2.1, le revêtement universel holomorphe riemannien de $\left(M, g^{\mathrm{K}}\right)$ donné par $\mathbb{H}^{2} \times \mathbb{C P}^{1}$, muni de sa métrique kählérienne standard à courbure scalaire constante $s=2(c-1)$.

Démonstration dans le cas où $s=0$. - On définit le nombre caractéristique

$$
\sigma=\int_{M} \gamma \quad \text { où } \quad \gamma=-\frac{1}{24 \pi^{2}}\left[\operatorname{tr}\left(R^{g^{\mathrm{K}}} \wedge R^{g^{\mathrm{K}}}\right)\right] .
$$

En dimension 4, on en déduit que

$$
\sigma=\frac{1}{12 \pi^{2}} \int_{M}\left|W^{+}\right|^{2}-\left|W^{-}\right|^{2} \mathrm{vol}^{g^{\mathrm{K}}},
$$

où $W^{ \pm}$sont les parties autoduales et anti-autoduales du tenseur de Weyl. Une surface kählérienne est anti-autoduale si et seulement si $s=0$. Maintenant, il est facile de calculer $\sigma$ : pour cela, on peut quitte à changer la structure holomorphe du fibré parabolique $\mathcal{E} \rightarrow \bar{\Sigma}$ supposer que c'est un fibré paraboliquement stable. On en déduit une métrique de Kähler $\hat{g}$ obtenue par construction standard à courbure scalaire $s=0$. Par la formule de transgression, $\sigma$ ne dépend que de la classe de cohomologie $L^{2}$ représentée par $\gamma$ donc

$$
\sigma=\int_{M} \gamma=\int_{M} \hat{\gamma} \quad \text { où } \quad \hat{\gamma}=-\frac{1}{24 \pi^{2}}\left[\operatorname{tr}\left(R^{\hat{g}} \wedge R^{\hat{g}}\right)\right] .
$$

Puisque $\hat{g}$ est un produit local de deux métriques de Kähler de courbure opposées sur des surface de Riemann, elle est donc conformément plate et d'après (30) il en résulte que $\sigma=0$. Finalement, on en déduit que $W^{-}=0$ pour la métrique $g^{\mathrm{K}}$ et qu'elle est elle aussi conformément plate. Or une surface kählérienne conformément plate est nćessairement un produit local de deux métriques kählériennes à courbures scalaires constantes opposées $\left(X_{1}, g_{1}\right) \times\left(X_{2}, g_{2}\right)$. En raison du comportement asymptotique de la métrique l'un des facteurs doit être $\mathbb{C P}^{1}$ et l'autre $\mathbb{H}^{2}$.

Démonstration dans le cas où $s<0$. - On commence par le lemme :

LEMme 14. - Sous les hypothèses du théorème 2.1 et en supposant que les équations de Seiberg-Witten non perturbées associées à la métrique $g=\xi^{*} g^{\mathrm{K}}$ ont une solution $(A, \psi)$ avec la régularité spécifiée dans le lemme 12 , on en déduit que le revêtement holomorphe riemannien de $M$ est égal à $\mathbb{H}^{2} \times \mathbb{C P}^{1}$ muni de sa métrique standard.

Remarque. - La fin de cet article a pour but de la démontrer l'existence de la solution $(A, \varphi)(c f . \S 4.3$ et $\S 4.4)$. 
La métrique de Kähler à courbure scalaire constante $g^{\mathrm{K}}$ induit une connexion $A^{\mathrm{K}}$ sur le fibré anti-canonique dont la courbure est harmonique et vérifie

$$
F_{A^{\mathrm{K}}}^{+{ }^{\mathrm{K}}}=-\frac{1}{4} i s \omega
$$

avec $\omega$ la forme de Kähler de $g^{\mathrm{K}}$; on en déduit que $\mathrm{L}^{2} c_{1}{ }_{g^{\mathrm{K}}}=\frac{s}{8 \pi}[\omega]$ d'où

$$
\left(\mathrm{L}^{2} c_{1}^{+{ }_{g} \mathrm{~K}}\right)^{2}=\frac{1}{32 \pi^{2}} \int_{M} s^{2} \mathrm{vol}^{g^{\mathrm{K}}} .
$$

Par définition $g=\xi^{*} g^{\mathrm{K}}$, donc par changement de variable

$$
\int_{M} s_{g}^{2} \mathrm{vol}^{g}=\int_{M} s^{2} \mathrm{vol}^{g^{\mathrm{K}}}
$$

en utilisant le corollaire 7

$$
\left(\mathrm{L}^{2} c_{1}^{+{ }_{g} \mathrm{~K}}\right)^{2}=\left(\mathrm{L}^{2} c_{1}^{+g}\right)^{2}=\frac{1}{32 \pi^{2}} \int_{M} s^{2} \mathrm{vol}^{g} .
$$

En appliquant le lemme 12 dans le cas d'égalité, on en déduit que $g$ est de Kähler relativement à une structure complexe $J$ compatible avec l'orientation de $M$. En prenant l'image réciproque de ces structures par $\xi$, il vient que $g^{\mathrm{K}}=\xi^{*} g$ est kählérienne relativement à une structure complexe $J_{1}=\xi^{*} J$ compatible avec l'orientation inverse de $M$. En notant $J_{0}$ la structure complexe originelle sur $M$ qui commute avec $J_{1}$ on définit un automorphisme de carré 1 de $T M$ donné par $J_{0} J_{1}$. On obtient une décomposition parallèle de $T M$ en $L_{0} \oplus L_{1}$ suivant les espaces propres de $J_{0} J_{1}$. Finalement le revêtement universel holomorphe riemannien de $(M, g)$ est un produit $\left(X_{1}, g_{1}\right) \times\left(X_{2}, g_{2}\right)$. Puisque la courbure scalaire de $g^{\mathrm{K}}$ est constante et que $s\left(x_{1}, x_{2}\right)=s_{1}\left(x_{1}\right)+s_{2}\left(x_{2}\right)$, on en déduit que $s_{1}$ et $s_{2}$ sont constantes. Maintenant, en raison du comportement asymptotique de $g^{\mathrm{K}}$ l'un des deux facteurs doit être à courbure sectionnelle -1 et l'autre à courbure sectionnelle $c$.

\subsection{Convergence des solutions des équations de Seiberg-Witten}

Notre objectif est maintenant d'obtenir une solution non réductible aux équations de Seiberg-Witten (28) pour une surface complexe réglée à bouts paraboliques, car c'est un outil central dans la démonstration du théorème 2.1. Dans cette optique, nous montrons que l'on sait faire converger une suite de solutions des équations de Seiberg-Witten perturbées (32) pour les métriques $g_{j}$, vers une solution des équations non perturbées (28) pour la métrique $g=\xi^{*} g^{\mathrm{K}}$. Plus généralement, le théorème de convergence 4.2 s'applique à toute métrique $g$ asymptotique au modèle local sur une surface complexe.

Nous supposerons pour donc pour l'instant que $M$ est une surface complexe à bouts paraboliques vérifiant la condition $\alpha_{2}-\alpha_{1} \in \mathbb{Q}$. Nous expliquerons au $\S 4.4$ comment se passer de cette hypothèse. Pour simplifier nous supposons en outre que $M$ possède exactement un bout parabolique, les démonstrations

TOME $130-2002-\mathrm{N}^{\mathrm{O}} 3$ 
s'étendant trivialement au cas de plusieurs bouts. Soit $g$ une métrique sur $M$ asymptotique au modèle local $\hat{g}$ et $g_{j}$ sont ses approximations lisses sur la compactification orbifold $\bar{M}=M \cup D$, où $D=\mathbb{C P}^{1} / \mathbb{Z}_{q}$.

4.3.1. Compactification du fibré $K_{M}^{-1}$. - Rappelons que près de $D$, on a revêtement local à $q$ feuillets de $\bar{M}$ donné par

$$
\begin{aligned}
p^{q}: I_{a} /\left\langle\tau^{q}\right\rangle \times \mathbb{C P}^{1} & \longrightarrow I_{a} /\langle\tau\rangle \times \mathbb{C P}^{1}, \\
(x+i y,[\tilde{u}: \tilde{v}]) & \longmapsto\left(x+i y,\left[\tilde{u} \mathrm{e}^{-i \alpha_{1} x}: \tilde{v} \mathrm{e}^{-i \alpha_{2} x}\right]\right),
\end{aligned}
$$

que l'on prolonge en un revêtement ramifié $p^{q}: \Delta_{a / q} \times \mathbb{C P}^{1} \rightarrow \overline{\mathcal{B}}$ tel que $p^{q}\left(\{0\} \times \mathbb{C P}^{1}\right)=D$.

Le fibré en droites complexes $[D]$ associé au diviseur $D$ tel que $c_{1}([D])$ est le dual de Poincaré de $D$, est défini classiquement à l'aide de deux ouverts de trivialisation, l'un au-dessus de $M$, l'autre au-dessus de $\Delta_{a / q} \times \mathbb{C P}^{1}$ (dans le cas des orbifolds, il faut se placer sur le revêtement local ramifié pour que la construction ait un sens). En utilisant les coordonnées $(\xi=x+i y,[\tilde{u}: \tilde{v}])$, on recolle ces deux trivialisations par la fonction de transition $\rho=\mathrm{e}^{i x}$ qui est invariante sous l'action de $\mathbb{Z}_{q}$ et définit ainsi un orbifibré sur $\bar{M}$. Notons que le fibré $[D]$ restreint à $M$ devient évidemment trivial.

On construit sur $[D]$ des connexions définies explicitement, en utilisant les notations de $\S 3.1$, par

$$
B_{j}=\mathrm{d}-i \chi_{j}\left(\partial_{t} \varphi_{j}\right) \mathrm{d} \theta
$$

Ceci définit bien une connexion lisse sur $[D]$ puisque $\partial_{t} \varphi_{j}=-1$ près de $[D]$ d'où $\rho B_{j} \rho^{-1}=d$ près de $D$. Alors la 2 -forme $\varpi_{j}=\frac{i}{2 \pi} F_{B_{j}}$ représente $c_{1}([D])$ et par construction, la suite de connexions $B_{j}$ converge sur tout compact de $M$ vers la connexion triviale.

Lemme 15. - La classe de Chern $L^{2}$ du fibré $K_{M}^{-1}$ se décompose en

$$
\mathrm{L}^{2} c_{1}=c_{1}\left(K_{\bar{M}}^{-1}\right)+c_{1}\left([D]^{-1}\right) .
$$

Plus précisément, la connexion $\hat{A}$ sur $K_{M}^{-1}$ s'écrit sous la forme $\hat{A}=C+a$, où a est une 1-forme $L_{1}^{2}(g)$ sur $M$ et $C$ est une connexion lisse sur le fibré $L=K_{\bar{M}}^{-1} \otimes[D]^{-1}$ dont la courbure vérifie près de $D$

$$
F_{C}=-i c \operatorname{vol}^{\mathbb{C P}}
$$

avec c la courbure de la métrique de Fubini-Study.

Démonstration. - Dans le revêtement $p^{q}: \Delta^{*} \times \mathbb{C P}^{1} \rightarrow \mathcal{B}$, la connexion de Chern sur le fibré anti-canonique se décompose en $A^{\Delta^{*}} \otimes A^{\mathrm{FS}}$ avec $A^{\Delta^{*}}$ et $A^{\mathrm{FS}}$ les connexions de Chern sur les fibrés tangents de $\Delta^{*}$ et $\mathbb{C P}^{1}$. Mais on a $A^{\Delta^{*}}=$ $\mathrm{d}-\partial \ln \left(|\mathrm{d} z|^{2}\right)=\mathrm{d}-\partial \ln \left(|z|^{2}\right)-\partial \ln \left(\ln ^{2}|z|\right)$ et le dernier terme est $L_{1}^{2} ;$ on en déduit le lemme en utilisant la formule de Poincaré-Lelong. 
4.3.2. Équations perturbées pour les métriques $g_{j}$. — La compactification $\bar{M}$ de $M$ est une surface complexe; elle est donc munie d'une structure $\operatorname{spin}^{c}$ canonique de fibré déterminant $L_{0}=K_{\bar{M}}^{-1}$ avec pour fibrés de spineurs

$$
W^{+}=\Lambda^{0,0} \bar{M} \oplus \Lambda^{0,2} \bar{M} \quad \text { et } \quad W^{-}=\Lambda^{0,1} \bar{M} .
$$

Notons que cette structure $\operatorname{spin}^{c}$ coïncide avec celle des équations non perturbée si on la restreint à $M$.

Chaque métrique $g_{j}$ sur $\bar{M}$ induit un opérateur de Dirac modulo le choix d'une connexion unitaire $A$ sur $L_{0}$; on définit alors les équations de SeibergWitten perturbées par

$$
\mathrm{D}_{A} \psi=0, \quad\left(F_{A}-F_{B_{j}}\right)^{+g_{j}}=q(\psi),
$$

où $\psi \in \Gamma\left(W^{+}\right)$. Le groupe de jauge $\mathcal{G}=\operatorname{Map}\left(\bar{M}, S^{1}\right)$ agit par

$$
f \cdot(A, \psi)=\left(A-2 \frac{\mathrm{d} f}{f}, f \psi\right)
$$

sur l'espace des solutions des équations de Seiberg-Witten. D'après le lemme 15, $\left[F_{A}-F_{B_{j}}\right]=-2 i \pi c_{1}\left(L_{0} \otimes[D]^{-1}\right)=-2 i \pi \mathrm{L}^{2} c_{1}$; c'est précisément pour cette raison que nous avons perturbé les équations et peut alors extraire une solution des équations de Seiberg-Witten non perturbées associées à la métrique $g$ grâce au théorème suivant.

ThÉORÈmE 4.2. - Soit $g$, une métrique asymptotique au modèle local sur $M$, et soit $g_{j}$ les métriques sur $\bar{M}$ qui approximent $g$. Soit $C$, la connexion de référence sur $L=L_{0} \otimes[D]^{-1}$ déduite de la connexion $\hat{A}$ à l'aide du lemme 15. Supposons donnée une suite $\left(A_{j}, \psi_{j}\right)$ de solutions des équations de SeibergWitten perturbées (32) associées aux métriques $g_{j}$. Alors, quitte à extraire une sous-suite et à faire des changements de jauge, les $\left(A_{j}, \psi_{j}\right)$ convergent au sens $C^{\infty}$ sur tout compact de $M$ vers une solution $(A, \psi)$ des équations de SeibergWitten non perturbées (28) sur $(M, g)$ telle que:

- $A=C+a$, où a est une 1 -forme $L_{1}^{2}(g)$, vérifiant $\mathrm{d}^{* g} a=0$.

- $\psi \in L_{1}^{2}(g)$.

4.3.3. Contrôle $C^{0}$. - Le premier pas dans la démonstration du théorème 4.2 est un résultat de contrôle $C^{0}$ uniforme sur $\psi_{j}$ :

LEMME 16. - Il existe une constante $K$ telle que pour tout $j$ et pour toute solution $\left(A_{j}, \psi_{j}\right)$ des équations de Seiberg-Witten perturbées associées à la métrique $g_{j}$, on ait $\left|\psi_{j}\right| \leq K$.

Pour le voir il faut analyser la courbure de $B_{j}$ qui est explicite.

LEMME 17. - La courbure de la connexion $B_{j}$ vérifie

$$
F_{B_{j}}=-i \chi_{j} \frac{\partial_{t}^{2} \varphi_{j}}{\varphi_{j}} \mathrm{~d} t \wedge \varphi_{j} \mathrm{~d} \theta+F_{j}^{b}
$$

TOME $130-2002-\mathrm{N}^{\mathrm{O}} 3$ 
avec $\left|F_{j}^{b}\right|$ bornée indépendamment de $j$.

Démonstration du lemme 16. - Par la formule de Lichnerowicz

$$
\mathrm{D}_{A_{j}}^{2} \psi_{j}=\nabla_{A_{j}}^{*} \nabla_{A_{j}} \psi_{j}+\frac{1}{4} s^{g_{j}} \psi_{j}+\frac{1}{2} F_{A_{j}}^{+} \cdot \psi_{j}
$$

puis en utilisant les équations de Seiberg-Witten, il vient

$$
\nabla_{A_{j}}^{*} \nabla_{A_{j}} \psi_{j}+\frac{1}{4} s^{g_{j}} \psi_{j}+\frac{1}{2} F_{B_{j}}^{+} \cdot \psi_{j}+\frac{1}{2} q\left(\psi_{j}\right) \psi_{j}=0 .
$$

Rappelons que d'après les lemmes 2 et 17 ,

$$
s^{g_{j}}=s_{b}^{g_{j}}-2 \chi_{j} \frac{\partial_{t}^{2} \varphi_{j}}{\varphi_{j}}, \quad F_{B_{j}}=-i \chi_{j} \frac{\partial_{t}^{2} \varphi_{j}}{\varphi_{j}} \mathrm{~d} t \wedge \varphi_{j} \mathrm{~d} \theta+F_{j}^{b},
$$

avec $s_{b}^{g_{j}}$ et $\left|F_{j}^{b}\right|$ uniformément bornées. Par ailleurs $\left(\mathrm{d} t \wedge \varphi_{j} \mathrm{~d} \theta\right)^{+{ }_{j}}$ agit par produit de Clifford sur $W^{+}$avec des valeurs propres $\pm i, \varphi_{j}^{-1} \partial_{t}^{2} \varphi_{j}$ reste bornée supérieurement mais pas inférieurement donc l'équation (33) peut s'écrire sous la forme

$$
0=\nabla_{A_{j}}^{*} \nabla_{A_{j}} \psi_{j}+P_{j} \psi_{j}+P_{j}^{b} \psi_{j}+\frac{1}{2} q\left(\psi_{j}\right) \psi_{j}
$$

où sur le bout parabolique

$$
P_{j}=-\frac{1}{2} \chi_{j} \frac{\partial_{t}^{2} \varphi_{j}}{\varphi_{j}} \psi_{j}-\frac{i}{2} \chi_{j} \frac{\partial_{t}^{2} \varphi_{j}}{\varphi_{j}}\left(\mathrm{~d} t \wedge \varphi_{j} \mathrm{~d} \theta\right)^{+{ }_{j}} \psi_{j}
$$

est un opérateur dont les valeurs propres sont 0 et $-\varphi_{j}^{-1} \chi_{j} \partial_{t}^{2} \varphi_{j}$. L'opérateur linéaire $P_{j}^{b}$ est uniformément borné. Il en résulte que la partie négative de ses valeurs propres de $P_{j}+P_{j}^{b}$ reste bornée inférieurement.

On peut maintenant démontrer le lemme en utilisant le principe du maximum : si $x_{0} \in \bar{M}$ un maximum local de $\left|\psi_{j}\right|^{2}$, alors $\Delta^{g_{j}}\left(\left|\psi_{j}\right|^{2}\right)\left(x_{0}\right) \geq 0$. Or d'après l'identité $\Delta^{g_{j}}\left(\left|\psi_{j}\right|^{2}\right)=2 \operatorname{Re}\left\langle\nabla_{A_{j}}^{*} \nabla_{A_{j}} \psi_{j}, \psi_{j}\right\rangle-2\left|\nabla_{A_{j}} \psi_{j}\right|^{2}$, on en déduit qu'il existe une constante $\kappa>0$ telle que

$$
0 \geq-\kappa\left|\psi_{j}\left(x_{0}\right)\right|^{2}+\frac{1}{2}\left\langle q\left(\psi_{j}\right) \psi_{j}\left(x_{0}\right), \psi_{j}\left(x_{0}\right)\right\rangle=-\kappa\left|\psi_{j}\left(x_{0}\right)\right|^{2}+\frac{1}{4}\left|\psi_{j}\left(x_{0}\right)\right|^{4},
$$

d'où $\left|\psi_{j}\left(x_{0}\right)\right|^{2} \leq 4 \kappa$.

4.3.4. Convergence des connexions. - Notons $C_{j}=A_{j} \otimes B_{j}^{-1}$ la suite de connexions définies sur $L$. On utilise la jauge donnée par la connexion $C$ de « référence » du lemme 15 et on écrit

$$
C_{j}=C+\beta_{j}+\mu_{j},
$$

où $\mu_{j}$ est une 1-forme $g_{j}$-harmonique, et $\beta_{j}$, une 1-forme orthogonale aux formes $g_{j}$-harmoniques.

BULletin DE LA SOCIÉtÉ MATHÉMATIQUE DE FRANCE 
Convergence de la partie harmonique. - D'après le théorème des coefficients universels, valable pour les CW-complexes et en particulier pour les orbifolds, $H^{1}(\bar{M}, \mathbb{Z})$ est un réseau de $H^{1}(\bar{M}, \mathbb{R})$ et finalement le quotient

$$
H^{1}(\bar{M}, \mathbb{R}) / H^{1}(\bar{M}, \mathbb{Z})
$$

est un tore compact. Par ailleurs, les composantes connexes du groupe de jauge $\operatorname{Map}\left(\bar{M}, S^{1}\right)$ agissent comme le réseau $i H^{1}(\bar{M}, \mathbb{Z})$ sur les classes de cohomologie de $i H^{1}(\bar{M}, \mathbb{R})$. On en déduit que quitte à faire agir le groupe de jauge sur les solutions des équations de Seiberg-Witten $\left(A_{j}, \psi_{j}\right)$, on peut supposer que les classes de cohomologie définies par $\mu_{j}$ restent bornées; quitte à extraire une sous-suite de $\left(A_{j}, \psi_{j}\right)$, on peut donc supposer que $\left[\mu_{j}\right]$ converge. D'après la proposition 3.2 , il en résulte que $\mu_{j}$ converge au sens $C^{\infty}$ sur tout compact de $M$, vers une forme $g$-harmonique $L^{2}$.

Convergence de la partie orthogonale aux formes harmoniques. - Pour commencer on peut quitte à faire des changements de jauge dans la composante connexe de l'identité, ce qui n'affecte pas l'argument précédent, on peut se placer dans une jauge de Hodge telle que $\mathrm{d}^{*_{j}} \beta_{j}=0$. Puisque $\left(A_{j}, \psi_{j}\right)$ est solution des équations de Seiberg-Witten,

$$
F_{C_{j}}^{+{ }_{j}}=\left(F_{C}+\mathrm{d} \beta_{j}\right)^{+_{j}}=q\left(\psi_{j}\right),
$$

d'où d ${ }^{+{ }_{j}} \beta_{j}=q\left(\psi_{j}\right)-F_{C}^{+{ }_{j}}$. Le lemme 16 donne alors une borne $C^{0}$ sur $q\left(\psi_{j}\right)$ et comme la connexion de référence $C$ vérifie $F_{C}=\lambda$ vol ${ }^{\mathbb{C P}}$ près de $D$, elle admet également une borne $C^{0}$; en utilisant le fait que le volume des métriques est uniformément borné par le lemme 3, on en déduit une borne uniforme sur $\left\|\mathrm{d}^{+{ }_{j}} \beta_{j}\right\|_{L^{2}\left(g_{j}\right)}$. Sur une variété compacte

$$
2\left\|\mathrm{~d}^{+j} \beta_{j}\right\|_{L^{2}\left(g_{j}\right)}^{2}=\left\|\mathrm{d} \beta_{j}\right\|_{L^{2}\left(g_{j}\right)}^{2} ;
$$

alors, d'après la proposition 3.3

$$
c\left\|\beta_{j}\right\|_{L^{2}\left(g_{j}\right)}^{2} \leq\left\|\mathrm{d} \beta_{j}\right\|_{L^{2}\left(g_{j}\right)}^{2} .
$$

Finalement, Par conséquent, $\left\|\beta_{j}\right\|_{L_{1}^{2}\left(g_{j}\right)}$ est uniformément borné et on peut extraire une limite faible sur tout compact $\beta \in L_{1}^{2}(g)$ de $\beta_{j}$ orthogonale aux formes $g$-harmoniques $L^{2}$ et vérifiant $d^{* g} \beta=0$.

En conclusion, les connexions $C_{j}=C+\mu_{j}+\beta_{j}$ convergent au sens $L_{1}^{2}$-faible sur tout compact de $M$ vers une connexion $A=C+a$ avec $a=\mu+\beta \in L_{1}^{2}(g)$. Par construction, $B_{j}$ converge vers la connexion triviale sur tout compact de $M$; on en déduit que $A_{j}$ converge également vers $C$ sur tout compact.

Convergence de la partie spineur. - Comme dans la preuve du lemme 16, on écrit la formule de Lichnerowicz sous la forme

$$
0=D_{A_{j}} \psi_{j}=\nabla_{A_{j}}^{*} \nabla_{A_{j}} \psi_{j}+\left(P_{j}^{b}+P_{j}\right) \psi_{j}+\frac{1}{2} q\left(\psi_{j}\right) \psi_{j}
$$

TOME $130-2002-\mathrm{N}^{\mathrm{O}} 3$ 
où $P_{j}^{b}$ est un opérateur uniformément borné, et $P_{j}^{b}$ un opérateur dont la partie négative des valeurs propres est uniformément minorée. Alors, il existe une constante $\kappa>0$ telle que $-\left\langle\left(P_{j}^{b}+P_{j}\right) \psi_{j}, \psi_{j}\right\rangle \leq \kappa\left|\psi_{j}\right|^{2}$; en intégrant sur $M$ la formule (34) contre $\psi_{j}$, on obtient le contrôle

$$
\left\|\nabla_{A_{j}} \psi_{j}\right\|_{L^{2}\left(g_{j}\right)}^{2} \leq \kappa\left\|\psi_{j}\right\|_{L^{2}\left(g_{j}\right)}^{2}-\frac{1}{4} \int_{M}\left|\psi_{j}\right|^{4} \mathrm{vol}^{g_{j}} \leq \kappa\left\|\psi_{j}\right\|_{L^{2}\left(g_{j}\right)}^{2} .
$$

Des bornes uniformes $C^{0}$ sur $\psi_{j}$ et sur le volume des métriques $g_{j}$, on déduit alors une borne uniforme sur $\left\|\nabla_{A_{j}} \psi_{j}\right\|_{L^{2}\left(g_{j}\right)}$. On peut donc extraire de $\psi_{j}$ une limite faible sur tout compact $\psi$ telle que $\psi, \nabla_{C} \psi \in L^{2}(g)$. Finalement, $(A, \psi)$ est obtenue comme une limite faible sur tout compact de solutions des équations de Seiberg-Witten perturbées $\left(A_{j}, \psi_{j}\right)$. Or $B_{j}$ tend vers la connexion triviale sur tout compact de $M$ il en résulte que $(A, \psi)$ est solution à la limite des équations de Seiberg-Witten non perturbées (28).

Une fois ces contrôles $L_{1}^{2}$ obtenus, un procédé classique de bootstraping utilisant l'ellipticité des équations de Seiberg-Witten modulo l'action du groupe de jauge montre que la convergence est en réalité $C^{\infty}$ sur tout compact de $M$ $(c f .[13])$.

4.4. Cas irrationnel. - Nous allons maintenant expliquer comment généraliser le théorème 2.1 dans le cas d'une métrique asymptotique à un modèle $\mathcal{B}_{a}^{\alpha_{1}, \alpha_{2}}$ dont les poids sont tels que vérifient $\alpha_{2}-\alpha_{1} \notin \mathbb{Q}$. Dans ce cas, le revêtement $p: I_{a} \times \mathbb{C P} \rightarrow \mathcal{B}$ ne se factorise pas ( $c f$. lemme 1 et corollaire 1$)$, la compactification naturelle du bout parabolique devrait être un quotient par $\mathbb{Z}$ sur lequel nous ne disposons pas d'un théorème de lìndice approprié pour développer une théorie de Seiberg-Witten ( $c f$. [10] pour les orbifolds).

Pour éviter ce problème, nous allons faire «bouger » les poids, afin d'obtenir des modèles $\hat{g}^{j}$ vérifiant la condition de rationalité et qui approximent $\hat{g}$ sur tout compact de $M$. En recollant $g$ aux différents modèles $\hat{g}^{j}$, nous en déduirons des approximations par des métriques $g^{j}$; par ce qui précède nous pourrons extraire des solutions des équations de Seiberg-Witten pour ces métriques que nous ferons converger à leur tour vers une solution des équations pour la métrique $g$.

4.4.1. Approximation dans le cas irrationnel. — Rappelons que la construction du modèle local se fait via le choix d'une métrique hermitienne

$$
h=\left(\begin{array}{cc}
|z|^{\alpha_{1}} & 0 \\
0 & |z|^{\alpha_{2}}
\end{array}\right)
$$

et il apparaît clairement que $\hat{g}$ dépend de façon $C^{\infty}$ du choix des poids sur un compact de $M$. On choisit donc une suite de poids $\left(\alpha_{1}^{j}, \alpha_{2}^{j}\right)$ qui tend vers $\left(\alpha_{1}, \alpha_{2}\right)$, telle que $\alpha_{2}^{j}-\alpha_{1}^{j} \in \mathbb{Q}$ et on note $\hat{g}^{j}$ le modèle local associé. En reprenant les notations de 3.1 .2 , on pose

$$
g^{j}=\left(1-\chi_{j}\right) g+\chi_{j} \hat{g}^{j}
$$

BUlletin DE LA SOCiÉtÉ MATHÉmATiQUe DE FRANCE 
On fait en sorte que les poids convergent suffisamment vite de sorte que les métriques $\hat{g}^{j}$ soient très proches de $\hat{g}$ au sens $C^{2}$ sur l'anneau $j \leq t \leq j+1$.

REMARQUE. - La métrique $g^{j}$ est évidemment asymptotique au modèle local $\hat{g}^{j}$ et tout ce qui concerne le cas rationnel s'applique à elle. Par ailleurs, d'après le corollaire 2 , on a les isomorphismes

$$
\mathrm{H}_{L^{2}\left(g^{j}\right)}^{*}(M) \simeq \mathrm{H}_{\mathrm{DR}}^{*}\left(\bar{M}^{j}\right) \simeq \mathrm{H}_{L^{2}(g)}^{*}(M),
$$

via lesquels $\mathrm{L}^{2} c_{1}(M, g)=\mathrm{L}^{2} c_{1}\left(M, g^{j}\right)=c_{1}\left(K_{\bar{M}^{j}}^{-1} \otimes\left[D_{j}\right]^{-1}\right)$.

4.4.2. Convergence des 1-formes harmoniques. - On a une inégalité de Poincaré uniforme pour les fonctions qui est l'analogue du lemme 7 :

LEMME 18. - Il existe une constante $c>0$, telle que pour toute métrique $g^{j}$ et toute fonction $f \in L_{1}^{2}\left(g^{j}\right)$ vérifiant $\int_{M} f \mathrm{vol}^{g^{j}}=0$ on ait

$$
\int_{M}|\mathrm{~d} f|^{2} \mathrm{vol}^{g^{j}} \geq c \int_{M}|f|^{2} \operatorname{vol}^{g^{j}} .
$$

Démonstration. - On refait la démonstration du lemme 7 à l'identique. Il n'y a que deux points à vérifier pour que les arguments s'appliquent bien : le lemme 3 est valable pour les métriques $g^{j}$ et les constantes qui interviennent dans le lemme 4 ne dépendent que de la constante $h_{0}$ qui est la même pour toutes les métriques $\hat{g}^{j}$, ce qui n'est pas difficile.

On déduit du lemme 18 par une démonstration presque identique à celle de la proposition 3.2 l'analogue suivant :

Proposition 4.3. - Soit $b \in \mathrm{H}_{L^{2}}^{1}(M)$ et $\gamma_{j}$ la suite de ses représentants $g^{j}$ harmoniques $L^{2}$. Alors $\gamma_{j}$ converge au sens $C^{\infty}$ sur tout compact de $M$, vers le représentant $g$-harmonique $L^{2}$ de $b$.

4.4.3. Inégalité de Poincaré pour les 1-formes. - Afin de généraliser les résultats de convergence obtenus pour les 1-formes dans le cas rationnel, on commence par montrer que la constante $T$ du lemme 9 peut être choisie indépendamment de la métrique $\hat{g}^{j}$. Notons $\hat{g}_{k}^{j}$ les approximations successives suivant la méthode exposée au $\S 3.1$ de chaque métrique $\hat{g}^{j}$, on a alors le lemme suivant :

LEMME 19. - Il existe une constante $T$ suffisamment grande telle que le lemme 9 soit vérifié pour toutes les métriques $g=\hat{g}, \hat{g}^{j}$ ou $\hat{g}_{k}^{j}$.

Démonstration. - La seule modification à apporter dans la démonstration du lemme 9 concerne l'argument utilisant les séries de Fourier : comme

$$
\left(\alpha_{2}^{j}-\alpha_{1}^{j}\right)=\frac{r_{j}}{q_{j}} \longrightarrow\left(\alpha_{2}-\alpha_{1}\right) \notin \mathbb{Q},
$$

TOME $130-2002-\mathrm{N}^{\mathrm{O}} 3$ 
ceci entraîne que $q_{j}$ explose; plus géométriquement, les orbites de $\mathcal{X}_{\theta}$ pour la métrique $g$ limite ne se referment pas et s'enroulent sur un tore. Nous allons donc raffiner l'argument en utilisant les séries de Fourier sur le tore.

En reprenant les notations de la démonstration du lemme 9 la métrique $g$ s'écrit en coordonnées locales sur le bout parabolique

$$
g=\mathrm{d} t^{2}+\varphi^{2}(t) \mathrm{d} \theta^{2}+\frac{4 / c}{\left(1+|u|^{2}\right)^{2}}|\mathrm{~d} u-i \alpha u \mathrm{~d} \theta|^{2}, \quad \text { où } \alpha=\alpha_{2}-\alpha_{1} .
$$

En utilisant les coordonnées polaires $u=\rho \mathrm{e}^{i \theta_{2}}$, on calcule

$$
g=\mathrm{d} t^{2}+\mathrm{e}^{-2 t} \mathrm{~d} \theta^{2}+\frac{4 / c}{\left(1+\rho^{2}\right)^{2}}\left(|\mathrm{~d} \rho|^{2}+\rho^{2}\left|\mathrm{~d} \theta_{2}-\alpha \mathrm{d} \theta\right|^{2}\right)
$$

et $\mathcal{X}_{\theta}=\partial_{\theta}+\alpha \partial_{\theta_{2}}$ - notons que $\left(\mathrm{d} \theta, \mathrm{d} \rho, \mathrm{d} \theta_{2}\right)$ est une base duale de $\left(\mathcal{X}_{\theta}, \partial_{\rho}, \partial_{\theta_{2}}\right)$. Les orbites de $\mathcal{X}_{\theta}$ s'enroulent sur les tores

$$
T\left(t_{0}, \rho_{0}\right)=\left\{\left(t, \rho, \theta, \theta_{2}\right) ; t=t_{0} \text { et } \rho=\rho_{0}\right\} .
$$

suivant lesquels on écrit la décomposition de $f$ en série de Fourier

$$
f=\sum_{k, \ell} c_{k, \ell}(t, \rho) \mathrm{e}^{i\left(k \theta+\ell \theta_{2}\right)}
$$

on en déduit que

$$
\mathcal{X}_{\theta} \cdot f=\left(\partial_{\theta}+\alpha \partial_{\theta_{2}}\right) \cdot f=\sum_{k, \ell} i c_{k, \ell}(k+\alpha \ell) \mathrm{e}^{i\left(k \theta+\ell \theta_{2}\right)} .
$$

La difficulté dans le cas où $\alpha$ est irrationnel provient du fait que l'ensemble des valeurs prises par $k+l \alpha$ s'accumule en 0 . Notons tout d'abord que

$$
\ell \rightarrow \infty \quad \text { et } k \rightarrow \infty \text { lorsque } k+\ell \alpha \rightarrow 0 \text {. }
$$

Remarque. - Dans le cas de la métrique $\hat{g}^{j}$, on a $\alpha_{2}^{j}-\alpha_{1}^{j}=\alpha^{j}=r_{j} / q_{j} \rightarrow$ $\alpha \notin \mathbb{Q}$. Alors, pour tout $K>0$, il existe un $\epsilon>0$ suffisamment petit, tel que pour $j$ suffisamment grand on ait $\left|k+\alpha^{j} \ell\right|^{2} \leq \epsilon$ implique $k, \ell \geq K$. En effet, si cette assertion était fausse, ceci impliquerait $\alpha \in \mathbb{Q}$, ce qui est contradictoire. On vérifiera en vertu de cette remarque que tout ce que nous dirons dans la suite de cette démonstration s'applique également aux métriques $\hat{g}^{j}$ et $\hat{g}_{k}^{j}$ en choisissant $j$ et $k$ suffisamment grands.

Maintenant

$$
\partial_{\theta_{2}} \cdot f=\sum_{k, \ell} i \ell c_{k, \ell} \mathrm{e}^{i\left(k \theta+\ell \theta_{2}\right)}
$$

et puisque $\mathrm{d}^{\mathbb{C P}^{1}} f=\partial_{\theta_{2}} \cdot f\left(\mathrm{~d} \theta_{2}-\alpha \mathrm{d} \theta\right)+\partial_{\rho} \cdot f \mathrm{~d} \rho$ est une somme de termes orthogonaux, on en déduit que que

$$
\left|\mathrm{d}^{\mathbb{C P}^{1}} f\right|^{2} \geq \frac{4}{c}(\rho+1 / \rho)^{2}\left|\partial_{\theta_{2}} \cdot f\right|^{2} \geq \frac{4}{c}\left|\partial_{\theta_{2}} \cdot f\right|^{2} .
$$

BULLETIN DE LA SOCIÉTÉ MATHÉMATIQUe DE FRANCE 
On décompose l'espace des fonctions sur le tore en l'espace des fonctions presque invariantes suivant $\mathcal{X}_{\theta}$ et son orthogonal

$$
\operatorname{Inv}_{\epsilon}=\left\langle\mathrm{e}^{i\left(k \theta_{1}+\ell \theta_{2}\right)} /|k+\alpha \ell|^{2} \leq \epsilon\right\rangle, \quad \operatorname{Inv}_{\epsilon}^{\perp}=\left\langle\mathrm{e}^{i\left(k \theta_{1}+\ell \theta_{2}\right)} / \epsilon<|k+\alpha \ell|^{2}\right\rangle .
$$

Pour tout $K>0$ il existe un $\epsilon$ suffisamment petit, tel que la décomposition de $f \in \operatorname{Inv}_{\epsilon}$ en série de Fourier s'écrive

$$
f=\sum_{k, \ell \geq K} c_{k, \ell} \mathrm{e}^{i\left(k \theta+\ell \theta_{2}\right)} .
$$

d'où

$$
\int_{N}\left|\partial_{\theta_{2}} \cdot f\right|^{2} \operatorname{vol}^{g_{t}} \geq K^{2} \int_{N}|f|^{2} \operatorname{vol}^{g_{t}}
$$

Revenons à l'inégalité (21) de la démonstration du lemme 9 :

$$
\begin{array}{r}
\int_{N}|\nabla \beta|^{2}+\operatorname{Ric}^{g}(\beta, \beta) \geq \int_{N}\left|\nabla_{\partial_{t}} \beta\right|^{2}+\left|\varphi^{-1} \mathcal{X}_{\theta} \cdot f\right|^{2}+\left|\varphi^{-1} \mathcal{X}_{\theta} \cdot f_{2}\right|^{2} \\
-4\left\langle f, \varphi^{-1} \mathcal{X}_{\theta} \cdot f_{2}\right\rangle+\left|\mathrm{d}^{\mathbb{C P}} f\right|^{2}+\left|\mathrm{d}^{\mathbb{C P}} f_{2}\right|^{2}
\end{array}
$$

on voit donc que pour $f \in \operatorname{Inv}_{\epsilon}$, le terme mixte $-4\left\langle f, \varphi^{-1} \mathcal{X}_{\theta} \cdot f_{2}\right\rangle$ est contrôlé à l'aide de $\left|\varphi^{-1} \mathcal{X}_{\theta} \cdot f_{2}\right|^{2}$ et $\left|\mathrm{d}^{\mathbb{C P}^{1}} f\right|^{2}$ d'après (35) et (36).

Supposons $f \in \operatorname{Inv}_{\epsilon}^{\perp}$. Alors

$$
\int_{N}\left|\mathcal{X}_{\theta} \cdot f\right| \operatorname{vol}^{g_{t}} \geq \epsilon \int_{N}|f|^{2} \operatorname{vol}^{g_{t}}
$$

d'où

$$
\int_{N}\left|\varphi^{-1} \mathcal{X}_{\theta} \cdot f\right| \operatorname{vol}^{g_{t}} \geq \epsilon \varphi^{-2} \int_{N}|f|^{2} \operatorname{vol}^{g_{t}}
$$

Puisque $\varphi^{-2}(t) \geq \mathrm{e}^{2 t} \rightarrow+\infty$, on en déduit que pour $t \geq T$, suffisamment grand, le terme mixte est contrôlé par $\left|\varphi^{-1} \mathcal{X}_{\theta} \cdot f\right|^{2}$ et $\left|\varphi^{-1} \mathcal{X}_{\theta} \cdot f_{2}\right|^{2}$.

On en déduit l'analogue de la proposition 3.3 :

Proposition 4.4. - Il existe une constante $c>0$ telle que pour toutes les métriques $g^{j}$, et toute 1 -forme $\beta \in L_{1}^{2}\left(g^{j}\right)$, orthogonale aux formes $g^{j}$-harmoniques $L^{2}$, on ait :

$$
\int_{M}|\mathrm{~d} \beta|^{2}+\left|\mathrm{d}^{*_{j}} \beta\right|^{2} \mathrm{vol}^{g^{j}} \geq c \int_{M}|\beta|^{2} \mathrm{vol}^{l^{j}} .
$$

TOME $130-2002-\mathrm{N}^{\mathrm{O}} 3$ 
4.4.4. Convergence des 2-formes harmoniques. - Soit une classe de cohomologie $b \in \mathrm{H}_{L^{2}}^{2}(M)$. À l'aide du lemme de Poincaré 5 , on commence par choisir un représentant $\gamma \in L^{2}(g)$ égal à $\lambda$ vol $\mathbb{C P}^{1}$ sur le bout parabolique. On en déduit une suite de représentants $\gamma_{j} \in L^{2}\left(g^{j}\right)$ via l'isomorphisme du du corollaire 2 tels que $\left\|\gamma_{j}\right\|_{L^{2}\left(g^{j}\right)}$ soit borné et $\gamma_{j}$ converge vers $\gamma$ au sens $C^{\infty}$ sur tout compact de $M$.

On peut écrire le représentant $g^{j}$-harmonique $w_{j} \in L^{2}\left(g^{j}\right)$ de $b$ sous la forme $w_{j}=\gamma_{j}+\mathrm{d} \beta_{j}$, où $\beta_{j} \in L_{1}^{2}\left(g^{j}\right)$ est une 1-forme orthogonale aux formes $g^{j}$-harmoniques $L^{2}$ qui vérifie $\mathrm{d}^{*_{j}} \beta_{j}=0$. Grâce à l'inégalité de Poincaré de la proposition 4.4, on en déduit par une démonstration similaire à celle de la proposition 3.4 :

Proposition 4.5. - Soit une classe de cohomologie $b \in \mathrm{H}_{L^{2}}(M)$. Soit $\Omega_{j}$ la suite de ses représentants $g^{j}$-harmoniques $L^{2}$. Alors $\Omega_{j}$ converge au sens $C^{\infty}$ sur tout compact de $M$ vers le représentant $g$-harmonique $L^{2}(g)$ de $b$.

Chaque métrique $g^{j}$ induit une décomposition des formes $g^{j}$-harmoniques $L^{2}$ en leur partie autoduale et anti-autoduale,

$$
\mathrm{H}_{L^{2}}^{2}(M)=\mathrm{H}_{L^{2}}^{+^{j}}(M) \oplus \mathrm{H}_{L^{2}}^{-j}(M) .
$$

On démontre également facilement l'analogue du corollaire 6

Corollaire 8. - Soit une classe de cohomologie $b \in \mathrm{H}_{L^{2}}^{2}(M), \pm^{j}$ les projecteurs de $\mathrm{H}_{L^{2}}^{2}(M)$ associés à la métrique $g^{j}$ et \pm ceux associés à $g$; alors $b^{+^{j}} \rightarrow b^{+}$et $b^{-j} \rightarrow b^{-}$lorsque $j$ tend vers l'infini.

4.4.5. Convergence des solutions des équations de Seiberg-Witten pour les métriques $g^{j}$. - Il faut commencer par fixer des connexions de référence $C^{j}$ convenables sur les fibrés

$$
L_{j}=K_{\bar{M}^{j}}^{-1} \otimes\left[D^{j}\right]^{-1}
$$

Soit $\hat{A}$ la connexion induite par $\hat{g}$ sur $K_{M}^{-1}$ et $\hat{A}^{j}$ celles induites par les métriques $\hat{g}^{j}$. Alors les connexion $\hat{A}^{j}$ convergent vers $\hat{A}$ au sens $C^{1}$ sur tout compact de $M$ par construction de $\hat{g}^{j}$. D'après le lemme 15 , on peut donc construire alors une suite de connexions $C^{j}$ qui converge sur tout compact $M$ vers la connexion $\hat{A}$, lisses sur $L_{j}$ et telles que $\left\|C^{j}-\hat{A}^{j}\right\|_{L^{2}\left(g_{j}\right)}$ et $\left\|F_{C^{j}}-F_{\hat{A}^{j}}\right\|_{L^{2}\left(g_{j}\right)}$ soient bornés.

On démontre alors l'analogue du théorème 4.2 suivant :

ThÉORÈme 4.6. - Soit $\left(A^{j}, \psi^{j}\right)$, une suite de solutions des équations de Seiberg-Witten non perturbées associées aux métriques $g^{j}$ telles que:

- $A^{j}=C^{j}+a^{j}$, ò̀ $a^{j} \in L_{1}^{2}\left(g^{j}\right)$ est en jauge de Hodge $\mathrm{d}^{{ }^{* j}} a^{j}=0$ et la partie $g^{j}$-harmoniques $L^{2}$ de $a^{j}$ est uniformément bornée en cohomologie.

- $\psi^{j} \in L_{1}^{2}\left(g^{j}\right)$ relativement à la connexion $C^{j}$. 
- $\psi^{j}$ est uniformément bornée en norme $C^{0}$.

Alors, quitte à extraire une sous suite, $\left(A^{j}, \psi^{j}\right)$ converge au sens $C^{\infty}$ sur tout compact de $M$ vers une solution $(A, \psi)$ des équations de Seiberg-Witten non perturbées pour la métrique $g$ telle que

- $A=C+a$, avec $\mathrm{d}^{*} a=0$ et $a \in L_{1}^{2}(g)$.

- $\psi \in L_{1}^{2}(g)$ relativement à $C$.

Démonstration. - Notons que tout d'abord que la borne $C^{0}$ du lemme 16 ne dépend pas non plus de $j$ pour les métriques $g_{k}^{j}$ car la courbure scalaire des métriques $g^{j}$ est uniformément bornée. Les hypothèses de régularité sur $\left(A^{j}, \psi^{j}\right)$ sont donc justement celles qu'on peut faire si $\left(A^{j}, \psi^{j}\right)$ a été obtenue à l'aide du théorème 4.2 .

Par ailleurs, on a une borne uniforme sur $\left[\mu^{j}\right]$, où $\mu^{j}$ est la partie $g^{j}$ harmonique de $a^{j}$ car on s'est ramené à un domaine fondamental du tore $H^{1}\left(\bar{M}^{j}, \mathbb{R}\right) / H^{1}\left(\bar{M}^{j}, \mathbb{Z}\right)$ afin de faire converger $\mu_{k}^{j}$. On peut quitte à extraire une sous-suite supposer que $\left[\mu^{j}\right]$ converge et en déduire que $\mu^{j}$ converge vers une forme $g$-harmonique $L^{2}$ grâce à la proposition 4.5 .

Il est facile de faire converger $\beta^{j}$ et $\psi^{j}$ (cf. th. 4.2) maintenant que nous disposons de l'inégalité de Poincaré de la proposition 4.4 et de connexions de référence $C^{j}$ adéquates.

4.5. Calcul de l'invariant de Seiberg-Witten. - Soit $M$ une surface complexe réglée obtenue à partir d'un fibré parabolique $\mathcal{E}$. Il nous reste prouver l'existence de solutions des équations de Seiberg-Witten sur les compactifications de $M$. Pour cela, on commence par choisir une métrique kählérienne particulière quitte à perturber la structure complexe sur $\bar{M}$ pour laquelle on sait que l'espace des module est constitué d'un point sous la condition $\operatorname{deg} L<0$. Comme nous somme dans le cas où $b_{2}^{+}=1$, l'invariant de Seiberg-Witten ne dépend pas de la métrique sous une «condition de chambre » (38) ce qui nous permet de trouver une solution des équations pour ces métriques.

4.5.1. Cas rationnel. - Si les poids de la structure parabolique vérifient la condition de rationnalité, on en déduit une compactification orbifold $\bar{M}$. Comme la condition de stabilité est générique, on peut quitte à perturber la structure holomorphe de $\mathcal{E}$ le supposer stable. On en déduit une métrique kählérienne $\hat{g}$ à courbure scalaire constante $s=2(c-1)<0$ définie sur $M$. Par construction, les approximations $\hat{g}_{j}$ de $\hat{g}$ sont kählériennes sur $\bar{M}$ et on la même classe de Kähler $[\omega]$. Comme $\hat{g}$ est kählérienne à courbure scalaire constante,

$$
\mathrm{L}^{2} c_{1} \cdot[\omega]=\frac{s}{8 \pi} \int \omega \wedge \omega
$$

donc $\operatorname{deg} L=\mathrm{L}^{2} c_{1} \cdot[\omega]<0$ ce entraîne que l'espace des modules des équations de Seiberg-Witten perturbées $(32) \operatorname{sur}\left(\bar{M}, \hat{g}_{j}\right)$ s'identifie à l'espace des modules

TOME $130-2002-\mathrm{N}^{\mathrm{O}} 3$ 
de couples $\left(A^{0,1}, \alpha\right)$ où $A^{0,1}$ est une structure holomorphe sur le fibré $L_{0}$ et $\alpha$ une section holomorphe du fibré trivial $L_{0} \otimes K_{\bar{M}}$ (voir [16]) ; la seule solution est le fibré $K_{\bar{M}}^{-1}$ avec $\alpha$ la section holomorphe standard du fibré trivial $\mathcal{O}_{\bar{M}}$. On en déduit que pour toute métrique $g$ sur $\bar{M}$ vérifiant

$$
\mathrm{L}^{2} c_{1}^{+g} \cdot[\omega]<0,
$$

les équations de Seiberg-Witten perturbées on un invariant $\mathrm{SW}(g)=1$.

Soit $g^{\mathrm{K}}$ une métrique kählérienne à courbure scalaire constante $s$ asymptotique au modèle local $\hat{g}$ sur $M$. En notant $\omega^{K}$ sa forme de Kähler,

$$
\int_{\mathbb{C P}^{1}} \omega^{\mathrm{K}}=\int_{\mathbb{C P}^{1}} \omega
$$

en raison du comportement asymptotique de $g^{\mathrm{K}}$; d'après (37),

$$
\frac{s}{8 \pi}=\frac{\mathrm{L}^{2} c_{1} \cdot[\omega]}{[\omega]^{2}}=\frac{\mathrm{L}^{2} c_{1} \cdot\left[\omega^{K}\right]}{\left[\omega^{K}\right]^{2}},
$$

ce qui implique $[\omega]=\left[\omega^{\mathrm{K}}\right]$.

Soit $g^{\mathrm{K}}$ une métrique kählérienne sur $M$ à courbure scalaire constante $s<0$ asymptotique au modèle local (dont les poids vérifient la condition de rationnalité) Notons $g=\xi^{*} g^{\mathrm{K}}$, où $\xi$ est l'antipodie sur $M$ définie au $\S 4.2$ et $g_{j}$ la suite d'approximations de $g$. D'après le corollaire 6 et le lemme 7

$$
\mathrm{L}^{2} c_{1}^{+_{j}} \longrightarrow \mathrm{L}^{2} c_{1}^{+_{g}}=\mathrm{L}^{2} c_{1}^{+{ }_{g} \mathrm{~K}},
$$

donc d'après (37) pour $j$ suffisamment grand,

$$
\mathrm{L}^{2} c_{1}^{+_{j}} \cdot[\omega]<0
$$

on en déduit que $\mathrm{SW}\left(g_{j}\right)=1$ et que les équations de Seiberg-Witten perturbées associées à la métrique $g_{j}$ admettent une solution $\left(A_{j}, \psi_{j}\right)$. En utilisant le théorème 4.2 , on en déduit la solution recherchée des équation de Seiberg-Witten non perturbées associées à la métrique $g=\xi^{*} g^{\mathrm{K}}$.

4.5.2. Cas irrationnel. - Dans le cas où le modèle local $\hat{g}$ ne vérifie plus la condition $\alpha_{2}-\alpha_{1} \in \mathbb{Q}$, on approxime la métrique $g=\xi^{*} g^{\mathrm{K}}$ par les métriques $g^{j}$ définies au $\S 4.4$ qu'on approxime à leur tour par des métriques lisses $g_{k}^{j}$ sur des compactifications orbifold $\bar{M}^{j}$ suivant le $\S 3.1$. D'après les corollaires 6 et 8 , en notant $\pm_{k}^{j}$ les projecteurs des décompositions de $\mathrm{H}_{L^{2}}^{2}(M)$ induites par les métriques $g_{k}^{j}$, on déduit que

$$
\mathrm{L}^{2} c_{1}^{+_{k}^{j}} \longrightarrow \mathrm{L}^{2} c_{1}^{+{ }_{g}}=\mathrm{L}^{2} c_{1}^{{ }_{g \mathrm{~K}}}
$$

pour $j$ et $k$ tendant vers l'infini. On en déduit, en utilisant le $\S 4.5 .1$ que les équations de Seiberg-Witten non perturbées associées à la métrique $g^{j}$ admettent une solution via le théorème 4.2 . On fait converger à nouveau cette 
suite vers une solution des équations pour la métrique $g=\xi^{*} g^{\mathrm{K}}$ en utilisant le théorème 4.6 .

\section{BIBLIOGRAPHIE}

[1] Beauville (A.) - Surfaces algébriques complexes, Astérisque, vol. 54, Soc. Math. France, 1978.

[2] Biquard (O.) - Fibrés paraboliques stables et connexions singulières plates, Bull. Soc. Math. France, t. 119 (1991), pp. 231-257.

[3] _ Métriques d'Einstein à cusps et équations de Seiberg-Witten, J. reine angew. Math., t. 490 (1997), pp. 129-154.

[4] Brun (C. LE) - Polarized 4-manifolds, extremal Kähler metrics, and Seiberg-Witten theory, Math. Res. Lett., t. 2 (1995), pp. 653-662.

[5] BRun (C. LE) \& Singer (M.) - Existence and deformation theory for scalar flat Kähler metrics on compact complex surfaces, Invent. Math., t. 112 (1993), pp. 273-313.

[6] Burns (D.) \& DE Bartolomeis (P.) - Stability of vector bundles and extremal metrics, Invent. Math., t. 92 (1988), pp. 403-407.

[7] Cerveau (D.), Ghys (E.), Sibony (N.) \& Yoccoz (J.-C.) - Complex dynamics and geometry, Panoramas et synthèses, vol. 8, Soc. Math. France, 1999.

[8] Donaldson (S.K.) - A new proof of a theorem of Narasihman and Seshadri, J. Diff. Geom., t. 18 (1983), pp. 269-277.

[9] Forster (O.) - Lecture on Riemann Surfaces, Graduate Texts in Mathematics, vol. 81, Springer-Verlag, 1981.

[10] KaWASAKI (T.) - The index of elliptic operators over $V$-manifolds, Nagoya Math., t. 84 (1981), pp. 135-157.

[11] Kronheimer (P.B.) \& Mrowka (T.S.) - Monopoles and contact structures, Invent. Math., t. 130 (1997), pp. 209-255.

[12] Mehta (V.B.) \& SeshadRI (C.S.) - Moduli of vector bundles of curves with parabolic structures, Math. Ann., t. 248 (1980), pp. 205-239.

[13] Morgan (J.W.) - Seiberg-Witten equations and applications to the topology of smooth 4-manifolds, Mathematical Notes, vol. 44, Princeton University Press, 1996.

[14] NARAsimhan (M.S.) \& SeshadRI (C.S.) - Stable and unitary vector bundles on a compact Riemann surface, Ann. Math., t. 82 (1965), pp. 540564 .

[15] SESHADRI (C.S.) - Fibrés vectoriels sur les courbes algébriques, Astérisque, vol. 96, Soc. Math. France, 1982.

[16] Witten (E.) - Monopoles and four-manifolds, Math. Res. Lett., t. 1 (1994), pp. 769-796.

TOME $130-2002-\mathrm{N}^{\mathrm{O}} 3$ 